\title{
MECHANICAL SIMULATION OF ARTICULAR CARTILAGE BASED ON EXPERIMENTAL RESULTS
}

\author{
A Thesis presented to \\ The Faculty of the College of Engineering \\ California Polytechnic State University, San Luis Obispo \\ In Partial Fulfillment \\ of the Requirements for the Degree \\ Master of Science in General Engineering \\ by \\ Kevin Matthew Stewart \\ June 2009
}


(C) 2009

Kevin Matthew Stewart

ALL RIGHTS RESERVED

ii 


\section{COMMITTEE MEMBERSHIP}

TITLE: $\quad$ Mechanical simulation of articular cartilage based on experimental results

AUTHOR: Kevin Matthew Stewart

DATE SUBMITTED: June, 2009

COMMITTEE CHAIR: Dr. Scott Hazelwood, Assistant Professor

COMMITTEE MEMBER: Dr. Stephen Klisch, Associate Professor

COMMITTEE MEMBER: Dr. Lanny Griffin, Professor and Department Chair 


\section{ABSTRACT}

TITLE: $\quad$ Mechanical simulation of articular cartilage based on experimental results

\section{AUTHOR: $\quad$ Kevin Matthew Stewart}

Recently, a constituent based cartilage growth finite element model (CGFEM) was developed in order to predict articular cartilage (AC) biomechanical properties before and after growth $[1,2]$. Previous research has noted limitations in the CGFEM such as model convergence with growth periods greater than 12 days [3]. The main aims of this work were to address these limitations through (1) implementation of an exact material Jacobian matrix definition using the Jaumann-Kirchhoff (J-K) method and (2) quantification of elastic material parameters based upon research findings of the Cal Poly Cartilage Biomechanics Group (CPGBG) [4]. The J-K method was successfully implemented into the CGFEM and exceeded the maximum convergence strains for both the "pushed forward, then differentiated" (PFD) and "differentiated, then pushed forward" (DPF) methods [3], while maintaining correct material stress responses. Elastic parameters were optimized for confined compression (CC), unconfined compression (UCC), and uniaxial tension (UT) protocols. This work increases the robustness of the CGFEM through the J-K method, as well as defines an accurate starting point for $\mathrm{AC}$ growth based on the optimized material parameters. 


\section{ACKNOWLEDGEMENTS}

I would like to thank Dr. Stephen Klisch and Dr. Scott Hazelwood for their guidance and for providing the opportunity to perform this research. I would also like to thank Pasquale Vena, the Laboratory of Biological Structure Mechanics (Politecnico Di Milano), and Dr. Robert Sah, Dr. Albert Chen, and Gregory Williams of the Cartilage Tissue Engineering Laboratory at The University of California, San Diego.

Finally, thank you to my parents for their help and support while I pursued my education. 


\section{Table of Contents}

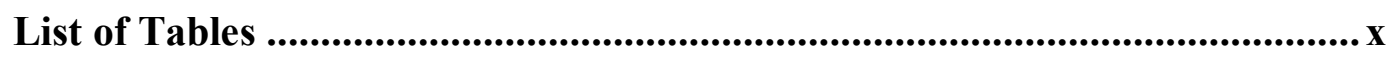

List of Figures .............................................................................................................. xi

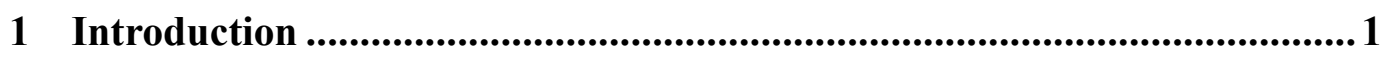

1.1 Articular Cartilage Background .......................................................... 1

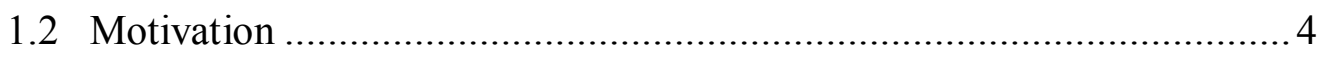

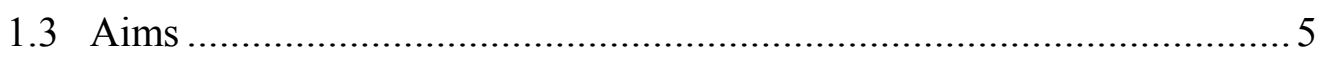

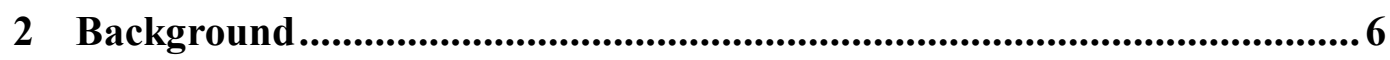

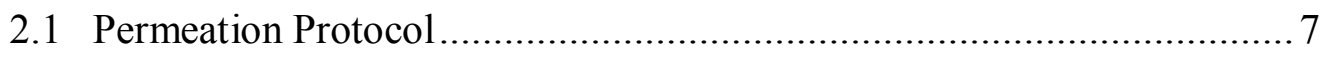

2.2 Experimental Property Determination ………………......................... 9

2.2.1 Confined Compression ............................................................ 10

2.2.2 Uniaxial Tension ................................................................. 11

2.2.3 Unconfined Compression........................................................ 13

2.3 Acknowledgements - Experimental ………………......................... 15

3 Theory ............................................................................................................... 16

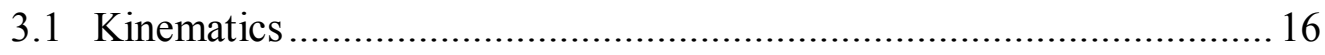

3.2 Elastic Constitutive Law …………………...................................... 19

3.2.1 Ground Substance Model........................................................... 20

3.2.2 Collagen Model .....................................................................2 20

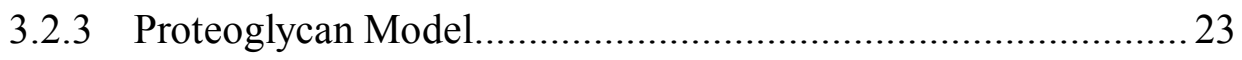




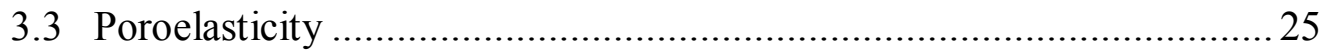

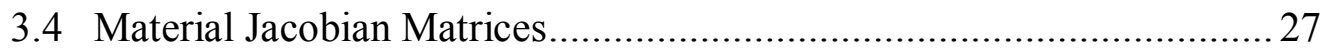

3.5 Acknowledgements - Theoretical......................................................... 32

$4 \quad$ J-K Stiffness Implementation ...........................................................................33

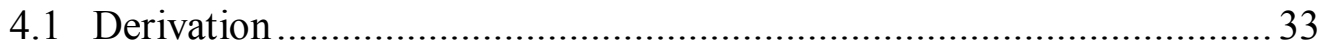

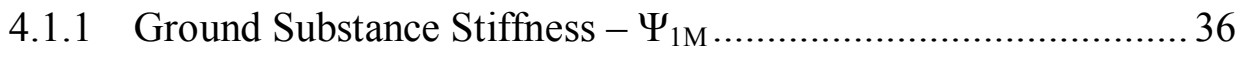

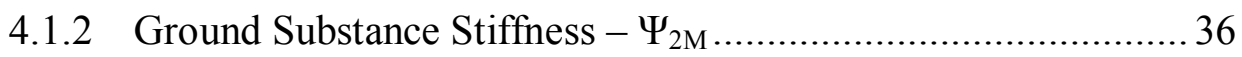

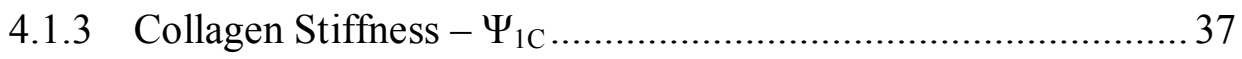

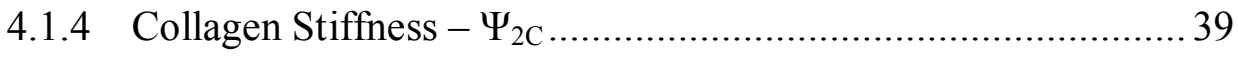

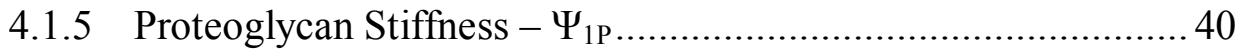

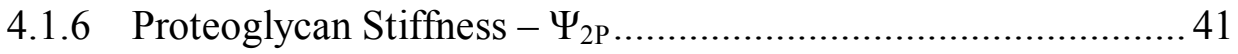

4.2 Implementation and Verification ....................................................... 43

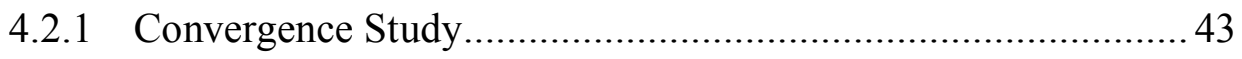

4.2.2 Validation Using Previous Models.............................................. 45

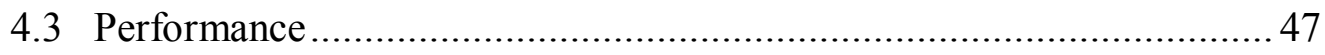

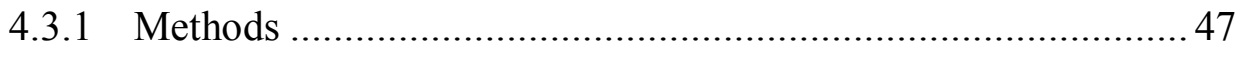

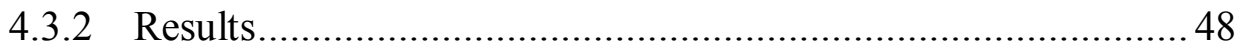

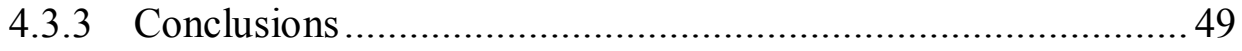

5 Quantifying Material Parameters ……............................................................51

5.1 Elastic Material Constant Overview ……………................................52

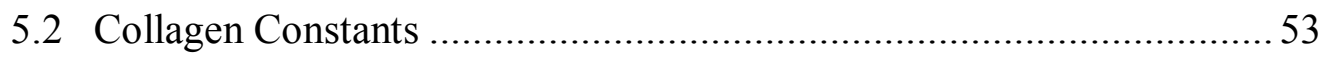

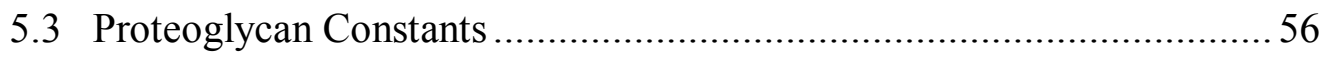


5.4 Solid Matrix Constants ............................................................. 59

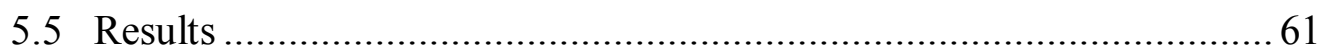

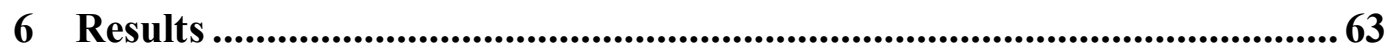

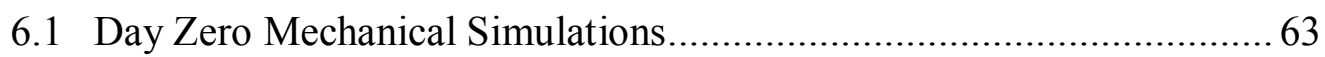

7 Discussion and Conclusions ................................................................6 67

List of References .................................................................................... 72

\section{Appendices}

A Derivations ............................................................................................ 77

A.1 Partial Derivative of a Tensor with Respect to its Inverse .................. 77

A.2 Linearization Analysis to Determine UCC Poisson's Ratio.................. 79

A.2.1 Collagen Constituent ..................................................... 79

A.2.2 Proteoglycan Constituent ............................................... 80

B Convergence Study.....................................................................83

C Initial Condition Iteration ..............................................................8 87

D Optimization Results...................................................................................8 89

D.1 MD vs. EXT2 Model in Simulating CC....................................... 89

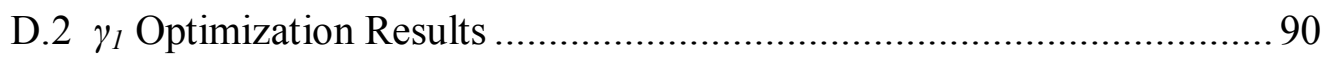

D. $3 \alpha_{2}$ Optimization Results.................................................. 92

D.4 $\mu$ Optimization Results with Error Analysis .................................. 93

E Codes and Inputs.........................................................................98

E.1 ABAQUS UMAT Files ...................................................... 98

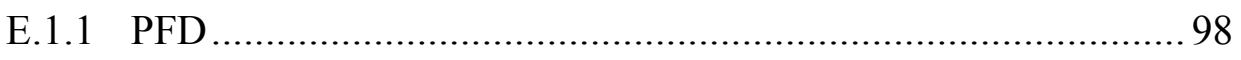

viii 


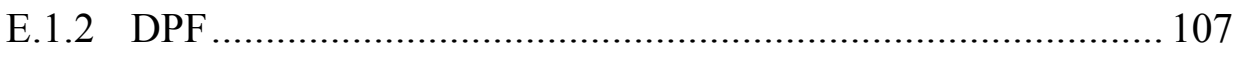

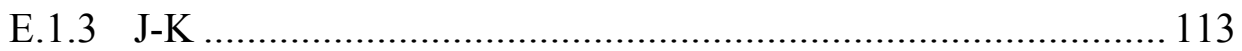

E.2 ABAQUS Input Files ......................................................... 120

E.2.1 Uniaxial Tension Elastic .................................................. 120

E.2.2 Unconfined Compression Elastic ........................................ 124

E.2.3 Confined Compression Elastic ........................................ 126

E.2.4 Poroelasticity Input File Sample (UT)............................. 128 


\section{List of Tables}

4.1 Collagen equation parameters used while verifying J-K method subroutine, and testing all subroutines for maximum convergence.

4.2 Proteoglycan equation parameters used while verifying J-K method subroutine, and testing all subroutines for maximum convergence.

5.1 Elastic constitutive law material constants.

5.2 Elastic constitutive law material constants resulting from parameter optimization studies.

6.1 Summary of experimental error between ABAQUS simulations and experimental results. Results are presented for all three material Jacobian matrix methods.

D.1 Experimental data for UT, CC, and UCC tests. This data provides the experimental comparison for all simulations.

D.2 Normalized CC 30\% and UT 10\% simulated results for variations of $\mu$ with SSE values shown.

D.3 Simulated data for UT, CC, and UCC using optimized parameters. 


\section{List of Figures}

2.1 A) Harvest location of bovine calf AC with physiological axes denoted by 1 (medial-lateral), 2 (anterior-posterior) , 3 (proximaldistal). B) Middle zone slices extracted from AC block. C) Final $3.2 \mathrm{~mm}$ disc punched.

2.2 A) The cartilage disc is placed in the permeation bioreactor and precompressed between the porous platens. B) Growth media is pumped through the bioreactor at a prescribed rate.

2.3 CC experimental setup. AC sample is submerged and compressed between two porous platens, allowing fluid flow out of both sides. Radial expansion is prevented by the confining chamber.

2.4 UT harvest orientation. The anterior-posterior, or 2 direction, provides the pull direction during testing.

2.5 A) UCC experimental setup with AC sample submerged between two nonporous platens with no radial confinement and compressive load applied. B) Radial expansion due to compression.

3.1 Solid matrix stress balance during mechanical loading. 
4.164 element cubic poroelastic model under $45 \%$ confined compression. The stress discontinuities are shown as the color change between elements.

4.2 Maximum convergence strains for PFD, DPF, and J-K simulations. Data is shown for uniaxial tension (UT), confined compression (CC), and unconfined compression (UCC).

7.1 CC experimental and simulated data after optimization. The Cauchy stress is given for the direction of loading. CC experimental data was taken from P00 samples.

7.2 UCC experimental and simulated data after optimization. The Cauchy stress is given for the direction of loading. UCC experimental data was taken from D00 samples.

B.1 Stress convergence for the elastic model in UT at $10 \%$ strain.

B.2 Stress convergence for the poroelastic model in UT at $10 \%$ strain.

B.3 Stress convergence for the elastic model in $\mathrm{CC}$ at $15 \%, 30 \%$, and $45 \%$ strains.

B.4 Stress convergence for the poroelastic model in $\mathrm{CC}$ at $15 \%, 30 \%$, and $45 \%$ strains. 
D.1 MD and EXT2 model simulation comparisons to CC D00 and P00 experimental results.

D.2 Comparison of varying $\gamma_{1}$ values to experimental CC data using MD model.

D.3 Comparison of varying $\gamma_{1}$ values to experimental UT data using MD model.

D.4 Comparison of varying $\alpha_{2}$ values to experimental CC data using MD model.

D.5 Comparison of varying $\mu$ values to experimental UT data using MD model.

D.6 Comparison of varying $\mu$ values to experimental CC data using MD model.

D.7 Summary of SSE data for UT $10 \%$ and CC $30 \%$ simulations for varying $\mu$ with polynomial curve fit. 


\section{Chapter 1}

\section{Introduction}

\subsection{Articular Cartilage Background}

Articular cartilage (AC) is a translucent tissue that covers the end of articulating bones [5] and serves as the load-bearing surface within synovial joints [6]. It has excellent lubrication and wear characteristics [7] that allow it to transmit high loads during joint rotation. $\mathrm{AC}$ is composed of a liquid phase and a solid matrix (SM) [8]. The liquid phase is composed of interstitial fluid, which accounts for $70 \%$ to $95 \%$ of the wet weight [9]. The remaining SM component is composed of chondrocyte cells, type II collagen (15-22\% by wet weight), proteoglycans ( $5 \%$ to $10 \%$ by wet weight), and other non-collagenous proteins [9]. The chondrocytes represent only $1 \%$ of the overall cartilage volume, but contribute to maintenance of the SM through cellular and matrix metabolism [10]. The 
collagen component forms a fibrillar meshwork that provides the majority of AC's tensile and shear properties [11, 12]. The negatively charged proteoglycan component provides a fixed charge density that resists compressive forces and allows the cartilage to swell through the attraction of water $[13,14]$. The proteoglycan component is considered to be composed of smaller glycosaminoglycan (GAG) chains.

$\mathrm{AC}$ is an avascular tissue, which inhibits its ability to repair [15]. The only mechanism for nutrient transfer to chondrocytes is by diffusion of nutrients through the SM. As a result of this avascularity and direct nutrient supply, damage or degeneration of AC can lead to chronic pain in the form of arthritis [16]. In the United States, 36 million people suffer from the effects of arthritis [10]. The far reaching effects of this disorder have provided a motivation to tissue engineer $\mathrm{AC}$ with the possibility of permanent replacement; however, a tissue engineering strategy for AC that yields consistent results has yet to be developed [10].

The heterogeneity of AC has made it increasingly difficult to duplicate the mechanical properties that it exhibits experimentally [17]. AC also exhibits nonlinear stress response in both tension and compression $[11,13]$ that increase the overall complexity of this material. Biphasic models have been used to computationally simulate these mechanical properties in order to account for both the fluid and SM contributions to stress [18]. These models are useful in providing a 
theoretical approximation of mechanical cartilage behavior that can be used to predict cartilage response under various loading conditions. The mechanical models, while useful, do not provide any information or approximations regarding AC growth. For this reason, separate growth models must be created in order to predict changes in the $\mathrm{AC}$ tissue during growth.

This desire to tissue engineer and theoretically model AC has led to the formulation of a cartilage growth finite element model (CGFEM) that combines growth and mechanical constitutive laws into a single simulation program [1]. The CGFEM utilizes previously developed constitutive laws for collagen and proteoglycan constituents to define the overall mechanical response $[19,20]$. Growth laws have been implemented based on experimental growth data gathered [2, 3]. The purpose of the CGFEM is to provide a basis for simulation of AC growth under a variety of mechanical stimuli. These growth models simulate, on a constitutive basis, the changes that occur in biochemical composition, mechanical properties, and geometries during growth. This is useful in that it provides preliminary results for possible laboratory experiments, serving as a guide for designing future experiments. Additionally, the results obtained from these simulations may aid in the development of in vitro grown $\mathrm{AC}$ for in vivo implantation, by providing preliminary results of various growth conditions. 


\subsection{Motivation}

Previous implementation of the CGFEM utilized approximations in the formulation of its constitutive laws, which has led to the inability of the model to converge after 12 days of simulated growth [3]. This does not allow for the user to simulate long-term growth protocols, which may be useful in the laboratory setting. These approximations have also led to the inability of model convergence at high strains for uniaxial tension (UT), confined compression (CC), and unconfined compression (UCC) experimental protocols [3]. This limitation in the model creates the inability to model high strain mechanical simulations of $\mathrm{AC}$, which may be desirable in future experiments.

Additionally, previous work has focused on the implementation of materials behaviors such as viscoelasticity [21], and poroelasticity, or the growth mechanisms themselves $[1,3]$. The performance of the model simulating day zero (D00) mechanical properties for UT, CC, and UCC has been approximated using data collected by other research groups; however, enough experimental data has recently been gathered by the Cal Poly Cartilage Biomechanics Group (CPCBG) to allow for determination of elastic parameters based on this data alone $[11,13$, $22,23]$. 


\subsection{Aims}

The first aim of this work is to implement a Jaumann-Kirchhoff (J-K) stiffness matrix approach based on the Jaumann rate of the Kirchhoff stress. This will influence the constitutive equations within the CGFEM. The J-K method should allow for increased maximum strain convergence during mechanical loading as well increase the length of growth simulations.

The second aim is to quantify the elastic material parameters utilized in the mechanical modeling of day zero (D00) parameters of AC, based on data gathered internally. Sufficient unpublished experimental data has been collected by the CPCBG to justify basing these parameters on those studies. Previous simulations have based their data on experimental properties gathered from other sources $[11,13,22,23]$. Due to the variations in mechanical and biochemical properties among different AC samples from different research groups, it is desirable to solely use data gathered internally. This allows for the same experimental protocols to be used for all experimentation and simulations, as well as similar sample sources to be used between the different experimental protocols. 


\section{Chapter 2}

\section{Background}

This section outlines the experimental protocols being modeled within the CGFEM, as well as the experimental motivation for this work. In order to accurately simulate $\mathrm{AC}$, various mechanical properties must first be determined experimentally. Strain-dependent tensile and compressive Young's Moduli (E), aggregate modulus $\left(\mathrm{H}_{\mathrm{A}}\right)$ and compressive Poisson's ratio $(v)$ are the bulk mechanical properties that can be determined from the following protocols. These provide the basic properties that the AC model must simulate in order to provide an accurate measure of $\mathrm{AC}$ mechanical response.

The experimental data collection was performed as a portion of this work, and as a precursor to the theoretical work. I collected the necessary data in conjunction with other members of the CPCBG at the Cartilage Tissue Engineering Laboratory at the University of California, San Diego over the past two years. 
The summarized data gathered from all of the experimental protocols discussed in this section can be found in Appendix D (Table D.1).

\subsection{Permeation Protocol}

As mentioned in Chapter 1, the CGFEM's goal is to design experimental growth protocols. One such design, a fluid permeation protocol, was developed within the CGFEM [3]. This protocol called for the harvest of bovine calf (1-3 weeks) AC blocks from the patellofemoral groove (Figure 2.1). Following block extraction, middle zone slices were obtained parallel to the surface $(\sim 0.75 \mathrm{~mm}$ depth from surface) and followed by punching the slice into a $3.2 \mathrm{~mm}$ disc. The discs were harvested with the articular surface being in the 3-direction, which was also the test axis.

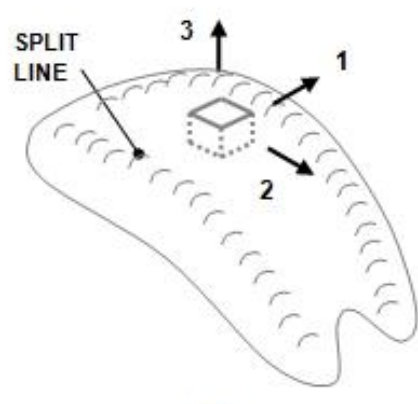

A

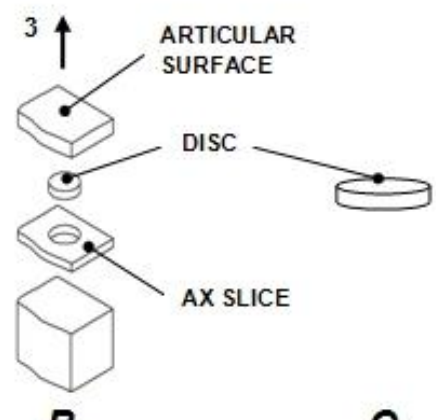

B

$c$

Figure 2.1: A) Harvest location of bovine calf AC with physiological axes denoted by 1 (medial-lateral), 2 (anterior-posterior), 3 (proximal-distal). B) Middle zone slices extracted from AC block. C) Final $3.2 \mathrm{~mm}$ disc punched. 
After being punched, the AC disc was placed in a permeation bioreactor (Figure 2.2) [24]. A pre-compression was applied to the permeation bioreactor to induce lateral expansion, thus sealing the cartilage against the outer wall. This pre-compression also prevented platen-specimen separation due to fluid diffusive drag forces during permeation. Growth media was then permeated through the disc over the course of 12 days using a pre-defined diffusion rate. A diffusion rate of $0.50 \mu \mathrm{m} / \mathrm{s}$ was used based on experimental results showing that this rate increased collagen and proteoglycan deposition $[3,25,26]$.

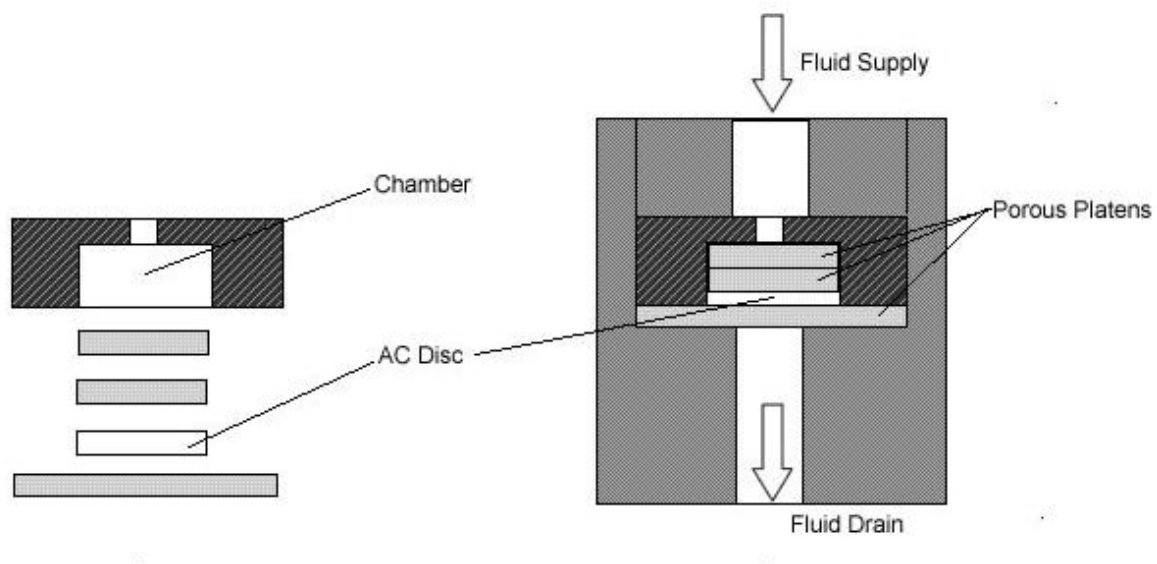

A

B

Figure 2.2: A) The cartilage disc is placed in the permeation bioreactor and precompressed between the porous platens. B) Growth media is pumped through the bioreactor at a prescribed rate.

Before (P00) and after (P12) growth, the AC disc was to be subjected to CC testing to determine mechanical properties. The D00 designation represents samples collected by members of the CPCBG that were not part of the permeation experi- 
mental sample group. P00 and P12 were samples that were part of the permeation experimental sample group. Biochemical properties, such as collagen, proteoglycan, and water content were also quantified pre and post growth using the CGFEM.

I implemented this permeation protocol with assistance from other members of the CPCBG in summer 2008. These pilot experiments were able to successfully perform the 12 day growth protocol as described in [3] and obtain biochemical and biomechanical data for both P00 and P12 groups. The experimental results were to be used to refine the CGFEM protocol developed by providing the experimental results that the CGFEM was approximating. The goal of this work is to provide an accurate starting point for simulation of permeation growth utilizing previously developed $\mathrm{AC}$ mechanical properties.

\subsection{Experimental Property Determination}

The elastic material properties of interest in this work can be determined from CC, UCC, and UT mechanical tests. I gathered CC data from permeation day zero tests (P00) with assistance from other members of the CPCBG during summer 2008. The permeation protocol did not call for any other mechanical testing to be performed. As a result, UT and UCC data was not gathered from the permeation samples, but instead completed using other unpublished data gathered by myself and other members of the CPCBG during the summer of 2008 . The UT 
and UCC day zero (D00) samples were harvested from the same knee specimens as the P00 samples. Since all samples were taken from the same knees, it was assumed that the mechanical properties would remain the same between experimental groups. Experiments have shown that there is little variation in material properties in samples taken from differing parts of the patellofemoral groove [11, 13].

\subsubsection{Confined Compression}

The CC test on the P00 samples was performed according to the CC protocol described previously [13] and the chamber transfer protocol developed in [3]. The chamber transfer protocol allowed for the sample to be pushed directly from the permeation growth chamber into the $\mathrm{CC}$ test chamber (Figure 2.3). This ensured that no lateral expansion due to growth occurred before $\mathrm{CC}$ testing was performed. The samples were ramped from $0 \%$ to $15 \%, 30 \%$, and $45 \%$ compressive strain levels and then allowed to reach equilibrium at each strain level. Once equilibrium was reached, a series of oscillatory compressions were applied to the sample. The stress is recorded at equilibrium, and using a finite deformation model [27], the secant aggregate modulus $\left(\mathrm{H}_{\mathrm{A}}\right)$ is determined. The measurements from the oscillations are then used to determine the permeability of the material at the tested strain levels [28]. 


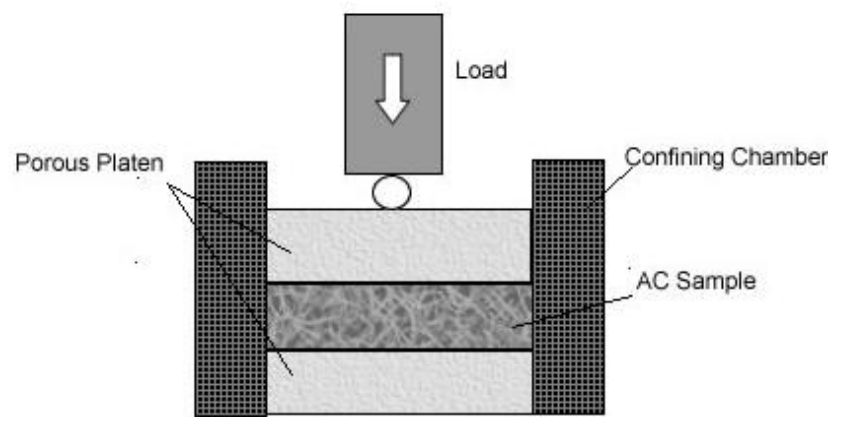

Figure 2.3: CC experimental setup. AC sample is submerged and compressed between two porous platens, allowing fluid flow out of both sides. Radial expansion is prevented by the confining chamber.

$\mathrm{H}_{\mathrm{A}}$ is directly affected by the collagen and proteoglycan solid matrix constituents [13] and provides the basis for comparison between the simulated experiments and the laboratory experiments.

\subsubsection{Uniaxial Tension}

For UT, blocks were harvested in the same method as described during permeation, with the exception of the punch shape. "Dog bone" shaped tensile coupons were punched from the middle zone slices according to established protocols (Figure 2.4) [11]. The previously mentioned protocol ramped the tensile coupon to a prescribed strain level and then allowed this to relax for $900 \mathrm{~s}$. 


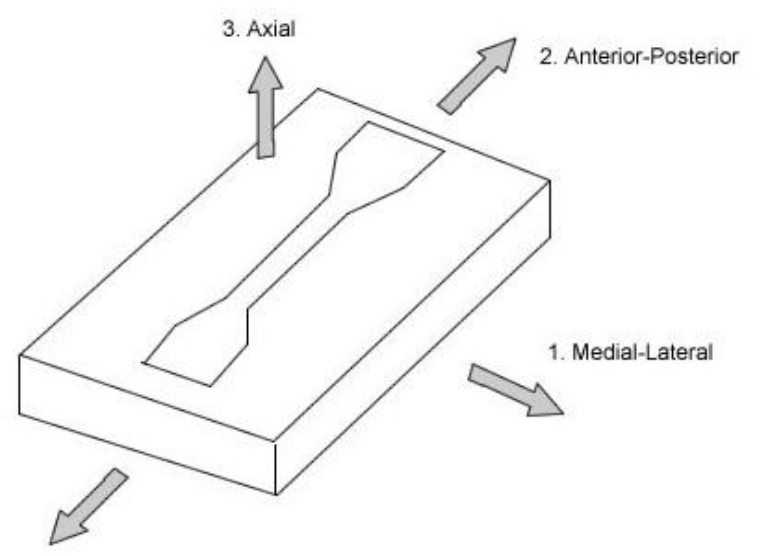

Figure 2.4: UT harvest orientation. The anterior-posterior, or 2 direction, provides the pull direction during testing.

It has been hypothesized that these specimens had not reached full relaxation at the $900 \mathrm{~s}$ mark [21]. For this reason, tensile data gathered by the CPCBG utilized a $5000 \mathrm{~s}$ relaxation time coupled with a novel equilibrium analysis program. This program extrapolated the $5000 \mathrm{~s}$ experimental data to determine the final equilibrium stress. This work utilized the UT data from the CPCBG since it is considered to more accurately predict the secant modulus at equilibrium. Strain levels of $5 \%$ and $10 \%$ were utilized for this study, since internal research has suggested that strain levels $>10 \%$ can lead to tissue damage and a resulting decrease in modulus.

The tensile data led to the calculation of the equilibrium secant tensile modulus, E. The equilibrium tensile modulus shows a strong correlation with col- 
lagen content [11]. As a result, the tensile data provides the main point to develop collagen parameters within the CGFEM.

\subsubsection{Unconfined Compression}

UCC specimens were harvested in a similar fashion as the CC specimens. UCC samples were tested according to the protocol in [29]. The UCC test compresses the sample axially between two nonporous platens, without any lateral confinement or equilibrium oscillations (Figure 2.5). UCC is used to measure the equilibrium Young's modulus (E), as well as the Poisson's ratio ( $v)$ of the cartilage in compression. When loading $\mathrm{AC}$ in the 3 direction under $\mathrm{UCC}$, the lateral expansion in the 1 and 2 directions has been shown to be not statistically different [29], supporting the findings that cartilage should be modeled as an anisotropic material [16]. Due to this, it is assumed that $v_{31} \approx v_{32}$. In the data collected by the CPCBG, $4.8 \mathrm{~mm}$ diameter UCC specimens were used as opposed to the 3.2 $\mathrm{mm}$ diameter specimens in the $\mathrm{CC}$ test. This larger diameter assisted with the measurement of the Poisson's ratio by decreasing edge effects that may distort Poisson's ratio readings. The protocol to gather this data is described in [29]. 


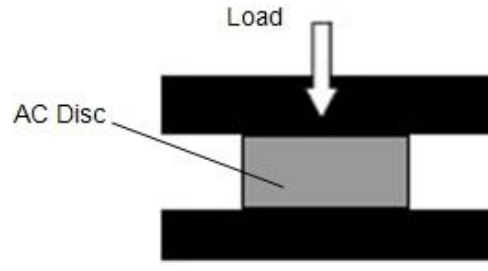

A

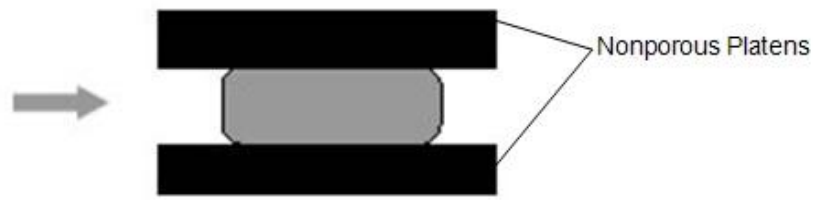

B

Figure 2.5: A) UCC experimental setup with AC sample submerged between two nonporous platens with no radial confinement and compressive load applied. B) Radial expansion due to compression.

Elastic modulus, E, has been shown to be positively correlated with both collagen and GAG content. Poisson's ratio, $v$, has been shown to be negatively correlated with collagen content, but showed no significant relationship with GAG content [29]. Based on these experimental findings, the CGFEM needs to be able to model both the Poisson's ratio and modulus accurately in order to capture the entire mechanical behavior of AC in UCC. The UCC data utilized for this work was taken from our collaborative research conducted by Stender et al. [4].

All of the previously discussed experimental protocols can be used to define day zero mechanical properties of $\mathrm{AC}$ as well as in vitro grown $\mathrm{AC}$ mechanical properties for compression and tension analyses. The CGFEM input parameters can be modified to simulate a one-time mechanical test, as with D00 or P00, or it can be modified to conduct an entire growth analysis if necessary. 


\subsection{Acknowledgements - Experimental}

I worked during the summers of 2007 and 2008 to gather much of the data that is used in this work. It would not have been possible to do this without the assistance of Dr. Robert Sah, Dr. Albert Chen, and Gregory Williams of the Cartilage Tissue Engineering Lab at the University of California, San Diego. Additionally, CPCBG students also played critical roles in performing these experiments. Thank you to David Dyk, Lauren Nelson, Kristin Dills, and Michael Stender for their assistance in the permeation apparatus design and implementation as well as UT, CC, and UCC data collection. 


\section{Chapter 3}

\section{Theory}

This chapter presents the theory underlying the mechanical simulations performed. It gives a brief introduction to kinematics and continuum mechanics, as well as detailed descriptions of the constitutive models used in mechanical simulations and growth routines.

\subsection{Kinematics}

Consider a continuous body $\beta$, with a reference configuration $\boldsymbol{\kappa}_{o}(\beta)$ at time $t_{o}$. The body experiences a deformation or motion described by the new configuration $\boldsymbol{\kappa}(\beta)$ at a time $t$. A unique point on the body has a position in the reference configuration of $\mathbf{X}$ at time $t_{o}$, and later a position in the modified configuration of $\mathbf{x}$ at time $t$. The motion of the entire body can then be described by specifying the change in position of the particle from $\boldsymbol{\kappa}_{o}$ to $\boldsymbol{\kappa}$ in terms of time. 


$$
\mathbf{x}=\chi(\mathbf{X}, t)
$$

The deformation gradient tensor, $\mathbf{F}$, maps between $\boldsymbol{\kappa}_{o}$ and $\boldsymbol{\kappa}$. It is defined as

$$
\mathbf{F}=\frac{\partial}{\partial \mathbf{X}} \chi(\mathbf{X}, t) .
$$

The determinant of $\mathbf{F}$ describes the volumetric change in $\beta$ when mapping between $\boldsymbol{\kappa}_{o}$ to $\boldsymbol{\kappa}$. This is known as the Jacobian, $J$.

$$
J=\operatorname{det}(\mathbf{F}) .
$$

The Jacobian will always be positive and can represent volumetric expansion for values greater than unity, volumetric contraction for values less than unity, and no change for values equal to unity.

The deformation gradient tensor, F, can be uniquely decomposed as

$$
\mathbf{F}=\mathbf{R} \mathbf{U}=\mathbf{R} \mathbf{V},
$$

where $\mathbf{U}$ is the left stretch tensor, $\mathbf{V}$ is the right stretch tensor, and $\mathbf{R}$ is the rotation tensor. This is accomplished through the polar decomposition theorem [30]. The Biot strain measure, $\mathbf{E}$, is defined in terms of the right stretch tensor as shown:

$$
\mathbf{E}=\mathbf{U} \mathbf{-} \mathbf{I}
$$


This is typically known as nominal engineering strain. The right Cauchy Green deformation tensor, $\mathbf{C}$, is related to the left stretch and deformation gradient tensor. It is defined by

$$
\mathbf{C}=\mathbf{U}^{2}=\mathbf{F}^{T} \mathbf{F}
$$

where ${ }^{T}$ is the transpose operator. The strain energy function, $W$, defines the amount of strain energy stored within a body after a prescribed deformation has been imposed. The strain energy function is used to derive the constitutive stressstrain relationship for a material as follows:

$$
\mathbf{S}=\frac{\partial W}{\partial \mathbf{E}}=2 \frac{\partial W}{\partial \mathbf{C}}
$$

Equation (3.1.7) relates the second Piola-Kirchhoff stress, $\mathbf{S}$, to a strain measure (Lagrangian strain: $\mathbf{E}=\mathbf{0 . 5}(\mathbf{C}-\mathbf{I})$ or $\mathbf{C})$. This relationship defines the basic constitutive behavior of a material. The second Piola-Kirchhoff stress has no physical interpretation, so it is often advantageous to convert this stress to a form with a physical interpretation. The following transformation gives Cauchy stress, T, and first Piola-Kirchhoff stress, $\mathbf{P}$.

$$
J \mathbf{T}=\mathbf{F S F}^{T}=\mathbf{P F}^{T}
$$

Cauchy stress represents the force applied in the deformed configuration divided by the deformed area, while first Piola-Kirchhoff stress represents the force applied in the deformed configuration divided by the undeformed area. 


\subsection{Elastic Constitutive Law}

The elastic constitutive law used in all simulations is based on the bimodular polyconvex fiber-reinforced anisotropic strain energy function for AC [20]. The bimodular property refers to the nonlinear relationship that appears in $\mathrm{AC}$ when undergoing transitions from compressive to tensile strains. The fiberreinforced structure of the model creates a general composite structure, with collagen fibers embedded in a solid matrix (SM). This particular model contains nine fiber directions that are used to emulate the fiber composition of AC. This also assists in modeling the anisotropy of the materials, allowing for different mechanical properties in each direction.

The model utilizes a constitutive stress balance in order to quantify the stress response of the material. The SM term is represented by an isotropic solid with collagen fiber reinforcement. A proteoglycan constituent term is added to this in order to model the compressive resistance of the AC. The stress balance in terms of second Piola-Kirchhoff stress is shown as:

$$
\mathbf{S}^{S M}=\mathbf{S}^{C O L}+\mathbf{S}^{P G}+\mathbf{S}^{M A T} .
$$

The total SM stress, $\mathbf{S}^{S M}$, is considered to be the summation of the collagen, $\mathbf{S}^{C O L}$, proteoglycan, $\mathbf{S}^{P G}$, and ground substance, $\mathbf{S}^{M A T}$, constituents. 


\subsubsection{Ground Substance Model}

In previous work, the $\mathbf{S}^{M A T}$ term was considered to be the isotropic contribution due to the collagen network $\left(\mathbf{S}^{O}\right)[3,21]$. This work has generalized the isotropic constituent to account for contributions from not only collagen, but other solid matrix sources as well (i.e. molecular energies) $[9,31]$. Using this new definition, the ground substance constituent can be defined in terms of the right Cauchy Green deformation tensor of the entire SM, C, the identity matrix, I, and the generalized material constant, $\mu$.

$$
\mathbf{S}^{M A T}=\mu\left(\mathbf{I}-\mathbf{C}^{-1}\right) .
$$

\subsubsection{Collagen Model}

The collagen stress term can also be written as the bimodular stress term $\left(\mathbf{S}^{B I M}\right)$. The bimodular component is inactive under compressive strains, but activated when exposed to tensile strains.

The bimodular portion of the collagen stress is composed of three primary collagen fiber families, and six secondary fiber families as discussed by Klisch [20]. The primary fiber families define collagen fibers lying in three mutually orthogonal directions: (1) parallel and (2) perpendicular to the split-line direction along the articular surface, and (3) perpendicular to the articular surface. These are defined by the basis vectors $\mathbf{E}_{1}, \mathbf{E}_{2}$, and $\mathbf{E}_{3}$ and are referred to as the principal 
fibers. Structural tensors $\mathbf{M}_{1}, \mathbf{M}_{2}$, and $\mathbf{M}_{3}$ are constructed as with an orthotropic material using the tensor dyadic product notation $(\otimes)$ :

$$
\mathbf{M}_{1}=\left(\mathbf{E}_{1} \otimes \mathbf{E}_{1}\right), \quad \mathbf{M}_{2}=\left(\mathbf{E}_{2} \otimes \mathbf{E}_{2}\right), \quad \mathbf{M}_{3}=\left(\mathbf{E}_{3} \otimes \mathbf{E}_{3}\right)
$$

Additionally, two fiber families are introduced in each of the three planes formed by the basis vectors $\mathbf{E}_{12}, \mathbf{E}_{23}$, and $\mathbf{E}_{13}$. These secondary fibers generate the crosslinking mechanism that occurs between the collagen fibers, and lie at complementary angles to one another. The secondary directions also represent collagen fibers lying at oblique angles relative to the basis coordinate system. The directions of each secondary fiber are defined by an angle $\left(\phi_{ \pm 12}, \phi_{ \pm 13}, \phi_{ \pm 23}\right)$ relative to the principal unit vectors. The secondary fiber directions are given as

$$
\begin{aligned}
& \mathbf{E}_{ \pm 12}=\cos \phi_{ \pm 12} \mathbf{E}_{1} \pm \sin \phi_{ \pm 12} \mathbf{E}_{2}, \\
& \mathbf{E}_{ \pm 13}=\cos \phi_{ \pm 13} \mathbf{E}_{1} \pm \sin \phi_{ \pm 13} \mathbf{E}_{3}, \\
& \mathbf{E}_{ \pm 23}=\cos \phi_{ \pm 23} \mathbf{E}_{2} \pm \sin \phi_{ \pm 23} \mathbf{E}_{3},
\end{aligned}
$$

in terms of the basis vectors. As was done for the primary fibers, structural tensors are then constructed for the secondary fibers. 


$$
\begin{aligned}
\mathbf{M}_{ \pm 12}= & \cos ^{2} \phi_{12} \mathbf{E}_{1} \otimes \mathbf{E}_{1} \pm \sin ^{2} \phi_{12} \mathbf{E}_{2} \otimes \mathbf{E}_{2} \\
& \pm \cos \phi_{12} \sin \phi_{12}\left(\mathbf{E}_{1} \otimes \mathbf{E}_{2}+\mathbf{E}_{2} \otimes \mathbf{E}_{1}\right) \\
\mathbf{M}_{ \pm 13}= & \cos ^{2} \phi_{13} \mathbf{E}_{1} \otimes \mathbf{E}_{1} \pm \sin ^{2} \phi_{13} \mathbf{E}_{3} \otimes \mathbf{E}_{3} \\
& \pm \cos \phi_{13} \sin \phi_{13}\left(\mathbf{E}_{1} \otimes \mathbf{E}_{3}+\mathbf{E}_{3} \otimes \mathbf{E}_{1}\right) \\
\mathbf{M}_{ \pm 23}= & \cos ^{2} \phi_{23} \mathbf{E}_{2} \otimes \mathbf{E}_{2} \pm \sin ^{2} \phi_{23} \mathbf{E}_{3} \otimes \mathbf{E}_{3} \\
& \pm \cos \phi_{23} \sin \phi_{23}\left(\mathbf{E}_{2} \otimes \mathbf{E}_{3}+\mathbf{E}_{3} \otimes \mathbf{E}_{2}\right)
\end{aligned}
$$

Previous work $[3,20]$ utilized a cubic strain energy function, $W^{B I M}$. This formulation has been modified to a quadratic strain energy function, which yields an approximately linear stress-strain response in UT [21]. Recent data gathered by the CPCBG has shown that newborn bovine $\mathrm{AC}$ exhibits a roughly linear stress response at low strains, thus making the linear stress-strain equation in terms of Lagrangian strain components (i.e. $\left.\left(\lambda_{2}^{2}-1\right)\right)$ a valid approximation. This is in contrast to adult tissue which typically exhibits a highly nonlinear response in tension. The following equation defines the roughly linear stress relationship for calf AC:

$$
\begin{aligned}
\mathbf{S}^{B I M}= & \gamma_{1}\left[\lambda_{1}\right]\left(\lambda_{1}^{2}-1\right) \mathbf{M}_{1}+\gamma_{2}\left[\lambda_{2}\right]\left(\lambda_{2}^{2}-1\right) \mathbf{M}_{2}+\gamma_{3}\left[\lambda_{3}\right]\left(\lambda_{3}^{2}-1\right) \mathbf{M}_{3} \\
& +\delta\left[\lambda_{ \pm 12}\right]\left(\lambda_{ \pm 12}^{2}-1\right) \mathbf{M}_{ \pm 12} \\
& +\delta\left[\lambda_{ \pm 13}\right]\left(\lambda_{ \pm 13}^{2}-1\right) \mathbf{M}_{ \pm 13} \\
& +\delta\left[\lambda_{ \pm 23}\right]\left(\lambda_{ \pm 23}^{2}-1\right) \mathbf{M}_{ \pm 23}
\end{aligned} .
$$

In equation (3.2.6), $\lambda_{1}, \lambda_{2}$, and $\lambda_{3}$ represent the squared stretches along the principal fiber directions. These values are found in the deformation tensor, $\mathbf{C}$, in the 
11,22 , and 33 locations, respectively. $\lambda_{ \pm 12}, \lambda_{ \pm 13}$, and $\lambda_{ \pm 23}$ represent the squared stretches in the secondary fiber directions and populate the remaining tensor locations in C. $\gamma_{1}, \gamma_{2}, \gamma_{3}$, and $\delta$ represent material constants that define the bimodular properties according to the following definitions:

$$
\gamma_{i}\left[\lambda_{i}\right]=\left\{\begin{array}{c}
\gamma_{i}>0 \text { if } \lambda_{i}>1 \\
0 \text { if } \lambda_{i}<1
\end{array}, \delta_{ \pm i j}\left[\lambda_{ \pm i j}\right]=\left\{\begin{array}{c}
\delta_{ \pm i j}>0 \text { if } \lambda_{ \pm i j}>1 \\
0 \text { if } \lambda_{ \pm i j}<1
\end{array}\right.\right.
$$

for $i, j=3$. Thus, if any collagen fiber is not in tension, it will turn off and not contribute to the stress.

\subsubsection{Proteoglycan Model}

The proteoglycan model is based on the two compartmental swelling pressure model for AC $[14,19,32]$. The proteoglycan stress, $\mathbf{S}^{\mathrm{PG}}$, shown in equation (3.2.1), can be represented as follows:

$$
\mathbf{S}^{P G}=\frac{-\alpha_{1}^{*}}{\left(J^{P G}\right)^{\alpha_{2}-1}} *\left(\mathbf{C}^{P G}\right)^{-1}=\frac{-\left(\alpha_{1}\left(\rho_{O}^{P G}\right)^{\alpha_{2}}\right)}{\left(\operatorname{det}\left(\mathbf{C}^{P G}\right)\right)^{\frac{1}{2}\left(\alpha_{2}-1\right)}} *\left(\mathbf{C}^{P G}\right)^{-1} .
$$

$J^{P G}$ represents the proteoglycan Jacobian. $\alpha_{1}$ and $\alpha_{2}$ represent material constants determined during the experimental data curve fitting process [14]. $\rho_{O}^{P G}$ represents the proteoglycan density in the $\mathrm{AC}$ in units of $\mathrm{g} / \mathrm{cm}^{3}$ and $\mathbf{C}^{\mathrm{PG}}$ is the proteoglycan right Cauchy-Green strain tensor. 
The proteoglycan, collagen, and ground substance components contribute to an overall stress balance as exhibited by equation (3.2.1). It is necessary in most simulations to begin from a stress-free SM configuration. The stress-free SM configuration, $\mathbf{S}_{O}^{S M}$, is achieved through the following manipulations $[19,21]$. Each constituent begins in its own reference configuration: $\kappa_{O}^{P G}, \kappa_{O}^{C O L}$, and $\kappa_{O}^{M A T}$. The individual reference configurations then have an initial deformation $\left(\mathbf{F}_{O}^{P G}, \mathbf{F}_{O}^{C O L}, \mathbf{F}_{O}^{S M}\right)$ applied to them in order to bring them to the SM reference configuration $\left(\kappa_{O}\right)$. The ground substance term utilizes the entire solid matrix deformation gradient $\left(\mathbf{F}_{O}^{S M}\right)$ since the ground substance is considered to be combination of collagen, proteoglycan, and other solid matrix effects. It is assumed that the reference configuration and the deformed configuration for the proteoglycan constituents are the same, leading to a deformation gradient, $\mathbf{F}_{O}^{P G}=\mathbf{I}$. This is due to the fact that the swelling pressure model for the proteoglycan constituent does not have a stress-free configuration and stays the same under a condition of no deformation. In order to account for this inherent stress within the proteoglycan components, the collagen components must stretch, creating an initial deformation gradient $\mathbf{F}_{O}^{C O L} \neq \mathbf{I}$. The constituents are then combined, leading to a stress free solid matrix reference configuration. Since the system is now in equilibrium, 
an overall deformation, F, can be applied. The constitutive deformation gradients can then be calculated using the following relations [33]:

$$
\mathbf{F}^{P G}=\mathbf{F F}_{o}^{P G} \quad, \quad \mathbf{F}^{C O L}=\mathbf{F F}_{o}^{C O L} \quad, \quad \mathbf{F}^{M A T}=\mathbf{F F}_{o}^{S M}
$$

The new deformation gradients can then be used to calculate the stress for each constituent, using equations (3.2.2), (3.2.6), and (3.2.8). This process can then be repeated for any number of deformations (Figure 3.1) [21].

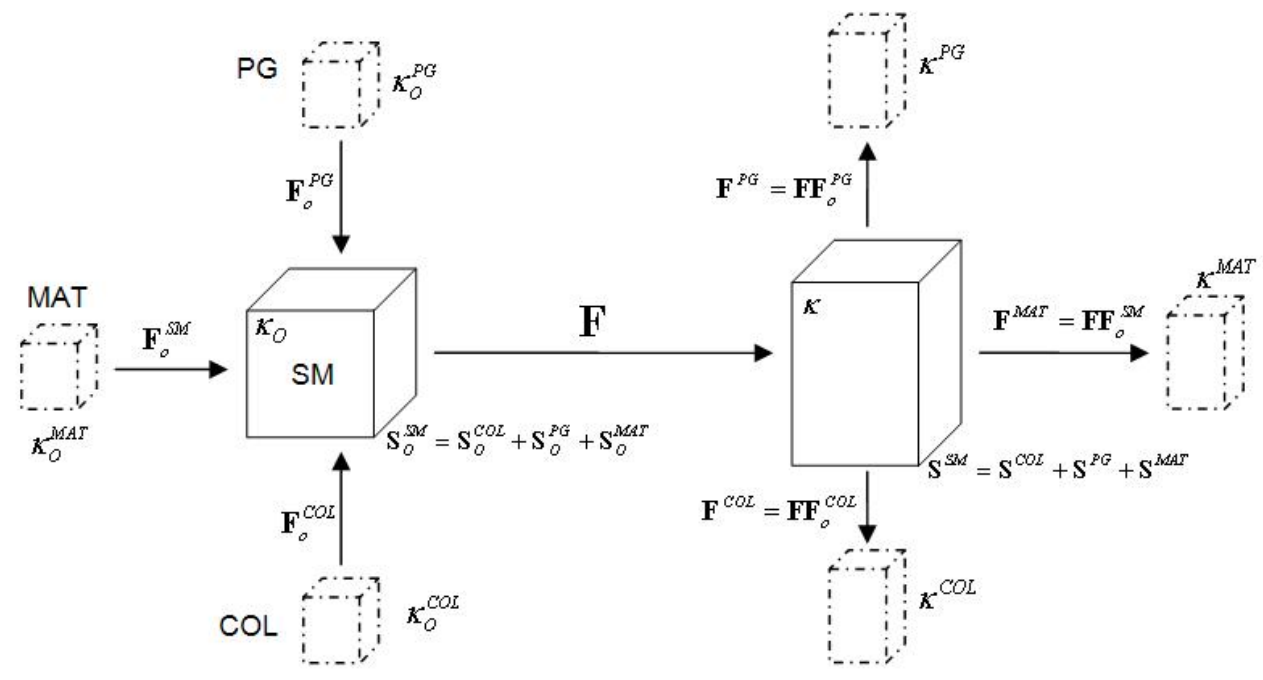

Figure 3.1: Solid matrix stress balance during mechanical loading.

\subsection{Poroelasticity}

The majority of this work deals with the elastic portion of the AC stress response, but for model validation purposes, a poroelastic simulation has been included. During compression of $\mathrm{AC}$, the interstitial fluid moves relative to the SM, creating localized pore pressures as well as diffusive velocities that work to 
equalize the pressure throughout the body. Linear biphasic mixture theory has successfully been applied to model the two phases of AC [8]. This poroelastic response model has been implemented into the current AC mechanical model [2, $3]$.

The biphasic mixture theory notes that the volume fraction of the SM, $\phi^{S M}$, and fluid, $\phi^{f}$, constituents must be constrained to obey a saturation constraint:

$$
\phi^{f}+\phi^{S M}=1 \text {. }
$$

The assumption of intrinsic incompressibility is also used, which assumes that the true densities are constant. The volume fraction is defined as

$$
\phi=\frac{\rho^{A}}{\rho^{T}},
$$

where $\rho^{A}$ is the apparent density and $\rho^{T}$ is the true density. The void ratio, $e$, is defined in terms of the fluid and SM volume fractions as

$$
e=\frac{\phi^{f}}{\phi^{S M}} .
$$

The permeability of the material is the measure of resistance to fluid flow through the porous SM. Using the permeability model developed by Ateshian, the permeability can be quantified as [34]: 


$$
k=k_{O}\left[\frac{e}{e_{O}}\right] \exp \left[\frac{M}{2}\left(\left\{\frac{1+e^{2}}{1+e_{O}^{2}}\right\}^{2}-1\right)\right]
$$

where $e_{O}$ is the initial void ratio, $e$ is the current void ratio, and $M$ is a nondimensional permeability coefficient. The permeability equation presented in equation (3.3.2) allows for the permeability to be updated as the void ratios changes. This allows for changes in permeability as the body is compressed or stretched, which has been shown to occur experimentally [35].

When poroelasticity is active, the overall stress is shared between the fluid and SM components:

$$
\mathbf{T}=\mathbf{T}^{f}+\mathbf{T}^{S M}
$$

In the model being used, the user material subroutine (UMAT) calculates the permeability, $k$, and SM Cauchy stress, $\mathbf{T}^{S M}$, while ABAQUS is used to calculate the coupled fluid and SM stresses. ABAQUS calls the UMAT to update the stresses based on the constitutive laws previously discussed.

\subsection{Material Jacobian Matrices}

The stiffness matrix forms the basic relation between stress and strain within the finite element analysis (FEA). ABAQUS utilizes a form of the stiffness matrix, called the material Jacobian matrix, that is specified within the UMAT [36]. ABAQUS utilizes the stiffness matrix to point the solver in the di- 
rection of the stress solution on an iteration by iteration basis. The stiffness matrix is defined as the partial derivative of stress increment with respect to the infinitesimal strain tensor:

$$
\widetilde{\mathbf{C}}=\frac{\partial \Delta \sigma}{\partial \Delta \varepsilon}
$$

ABAQUS defines the total exact stiffness matrix as

$$
\widetilde{\mathbf{C}}=\frac{1}{J} \frac{\partial J \mathbf{T}}{\partial \varepsilon^{L}},
$$

where, $\mathbf{T}$ represents the Cauchy stress, $J$ is the Jacobian, $\varepsilon^{L}$ is the logarithmic strain, and $\widetilde{\mathbf{C}}$ is the exact Jacobian matrix. $\widetilde{\mathbf{C}}$ is equivalent to a stiffness matrix within the FEA protocol and serves as the governing relationship between stress and strain. Previous work has utilized approximations of the total form of $\widetilde{\mathbf{C}}$ in equation (3.4.2)

It is common to approximate the stiffness matrix due to its nontrivial derivation when applied to certain material models. Previous work has approximated $\widetilde{\mathbf{C}}$ with success using "pushed forward, then differentiated" (PFD) and "differentiated, then pushed forward" (DPF) methods [3]. These models have yielded acceptable strain convergence, but could be improved upon using an exact derivation. A major goal of this work is to develop and implement an exact derivation of the stiffness matrix, using the J-K Method. 
The J-K Method, as developed by Vena [33, 37, 38], utilizes the Jaumann stress rate of the Kirchhoff stress. This gives a more generalized form of the stiffness equation than equation (3.4.2) that can be applied to the $\mathrm{AC}$ material model. Additionally, the J-K method gives terms that are correct for the rotational motion under deformations. These correctional rotation terms increase the J-K method's convergence in problems exhibiting co-rotations. The J-K method also uses the Kirchhoff stress relationship shown, where $\tau_{\mathrm{ij}}$ is the Kirchhoff stress, $\sigma_{\mathrm{ij}}$ is the Cauchy stress, $\boldsymbol{S}_{k h}$ is the second Piola-Kirchhoff stress, and $J$ is the Jacobian [38].

$$
\tau_{i j}=J \sigma_{i j}=F_{i k} S_{k h} F_{j h}
$$

The total increment of the Kirchhoff stress is then defined as

$$
\delta \tau_{i j}=\delta F_{i k} S_{k h} F_{j h}+F_{i k} S_{k h} \delta F_{j h}+F_{i k} \delta S_{k h} F_{j h} .
$$

The $\delta F_{i j}$ portion of equation (3.4.4), is developed using the rate of deformation (D), as well as the displacement increment gradient (L). D represents the symmetric portion of the displacement increment gradient, $\mathbf{L}$.

$$
L_{i j}=\frac{\partial \delta u_{i}}{\partial x_{j}} \quad, \quad D_{p q}=\frac{1}{2}\left(L_{p q}+L_{q p}\right)
$$


A generic fourth order elasticity tensor initially discussed can be used to define the partial derivative of the second Piola-Kirchhoff stress in terms of the rate of deformation and the deformation gradients. Using the relations

$$
\delta S_{a b}=C_{a b c d} \delta E_{c d} \quad \text { and } \quad \delta E_{c d}=F_{k c} D_{k l} F_{l k}
$$

one obtains:

$$
\delta S_{A B}=C_{A B C D} F_{k C} D_{k l} F_{l D},
$$

With the elasticity tensor shown in indicial notation. Note that in indicial notation, capital letter indices refer to the reference configuration, while lower case indices refer to the deformed configuration. The combination of equations (3.4.4), (3.4.5), and (3.4.7) leads to an overall equation for the Kirchhoff increment of:

$$
\delta \tau_{i j}=L_{i C} \tau_{C j}+L_{j A} \tau_{i A}+C_{A B C D} F_{i A} F_{j B} F_{k C} F_{l D} D_{k l} .
$$

This can be rearranged as

$$
\begin{aligned}
\delta \tau_{i j}= & \frac{1}{2}\left[\delta_{l i} \tau_{k j}+\delta_{k j} \tau_{i l}+\delta_{k i} \tau_{l j}+\delta_{l j} \tau_{i k}\right]+C_{A B C D} F_{i A} F_{j B} F_{k C} F_{l D} D_{k l}, \\
& +\omega_{i C} \tau_{C j}+\omega_{j A} \tau_{i A}
\end{aligned}
$$

where $\boldsymbol{\omega}$ is the incremental spin tensor. The Jaumann stress rate is defined as

$$
\delta^{J} \tau_{i j}=J^{-1} \widetilde{C}_{i j k l} D_{k l}=\delta \tau_{i j}-\omega_{i C} \tau_{C j}-\omega_{j A} \tau_{i A}
$$


Jaumann stress rate is an objective stress rate also known as the co-rotational stress rate. Since ABAQUS requires the use of an objective stress rate, Jaumann stress rate is a valid option. Combining equations (3.4.9) and (3.4.10) leads to the definition of the new tangent stiffness matrix:

$$
\widetilde{C}_{i j k l}=\frac{1}{J}\left\{\frac{1}{2}\left[\delta_{l i} \tau_{k j}+\delta_{k j} \tau_{i l}+\delta_{k i} \tau_{l j}+\delta_{l j} \tau_{i k}\right]+F_{i A} F_{j B} F_{k C} F_{l D} C_{A B C D}\right\}
$$

The J-K method tangent stiffness matrix provides the basis for the new work. Since ABAQUS typically outputs stresses in terms of Cauchy stress, equation (3.4.11) can also be manipulated as follows to be in terms of Cauchy stress $(T)$ :

$$
\widetilde{C}_{i j k l}=\frac{1}{J}\left\{\frac{J}{2}\left[\delta_{l i} T_{k j}+\delta_{k j} T_{i l}+\delta_{k i} T_{l j}+\delta_{l j} T_{i k}\right]+F_{i A} F_{j B} F_{k C} F_{l D} C_{A B C D}\right\} .
$$

Additionally, this new matrix must be applied for both the collagen and proteoglycan constituents. The details of the constitutive matrices can be found in Chapter 4.

The overall $\widetilde{C}_{i j k l}$ fourth-order tensor defined in equation (3.4.12) can be considered to have minor symmetries

$$
\widetilde{C}_{i j k l}=\widetilde{C}_{j k l}=\widetilde{C}_{i j k} .
$$

Using these symmetries, the $\widetilde{C}_{i j k l}$ tensor contains only 36 unique components of the 81 total components found in the tensor. Due to this, the fourth-order tensor, 
$\widetilde{C}_{i j k l}$, can be collapsed into a six-by-six matrix that only contains the 36 unique terms:

$$
\widetilde{C}_{i j k l}=\left(\begin{array}{cccccc}
C_{1111} & C_{1122} & C_{1133} & 2 C_{1112} & 2 C_{1113} & 2 C_{1123} \\
C_{2211} & C_{2222} & C_{2233} & 2 C_{2212} & 2 C_{2213} & 2 C_{2223} \\
C_{3311} & C_{3322} & C_{3333} & 2 C_{3312} & 2 C_{3313} & 2 C_{3323} \\
2 C_{1211} & 2 C_{1222} & 2 C_{1233} & 2 C_{1212} & 2 C_{1213} & 2 C_{1223} \\
2 C_{1311} & 2 C_{1322} & 2 C_{1333} & 2 C_{1312} & 2 C_{1313} & 2 C_{1323} \\
2 C_{2311} & 2 C_{2322} & 2 C_{2333} & 2 C_{2312} & 2 C_{2313} & 2 C_{2323}
\end{array}\right) .
$$

The scalars found within the matrix arise from the combination of terms using the collapsing convention. This matrix satisfies the form of the material stiffness matrix that is required by ABAQUS.

\subsection{Acknowledgements - Theoretical}

This work uses research conducted by previous members of the CPCBG.

I would like to acknowledge Michael Bingham [1], Tim Ficklin [3], Sevan Oungoulian [14], and Gregory Thomas [21] for providing a theoretical basis, as well as some of the code utilized in this work. Additionally, thank you to Pasquale Vena for outlining the Jaumann Kirchoff method derivation [33]. 


\section{Chapter 4}

\section{J-K Stiffness Implementation}

As discussed, previous work has used an approximation of the material stiffness matrix, while the J-K method applies an exact solution to the material stiffness matrix [3]. This chapter outlines the steps taken to apply the J-K method equation shown in equation (3.4.12) to both the collagen and proteoglycan constituents.

\subsection{Derivation}

The derivation necessary for implementation of the $\mathrm{J}-\mathrm{K}$ method has been partially completed through the approximation analyses [3]. The overall J-K method material stiffness matrix is repeated for convenience here 


$$
\widetilde{C}_{i j k l}=\frac{1}{J}\left\{\frac{J}{2}\left[\delta_{l i} T_{k j}+\delta_{k j} T_{i l}+\delta_{k i} T_{l j}+\delta_{l j} T_{i k}\right]+F_{i A} F_{j B} F_{k C} F_{l D} C_{A B C D}\right\} .
$$

The overall stiffness can be divided as follows for clarity during the derivation:

$$
\begin{gathered}
\Psi_{1}=\frac{J}{2}\left[\delta_{l i} T_{k j}+\delta_{k j} T_{i l}+\delta_{k i} T_{l j}+\delta_{l j} T_{i k}\right], \\
\Psi_{2}=F_{i A} F_{j B} F_{k C} F_{l D} C_{A B C D}, \\
\widetilde{C}_{i j k l}=\frac{1}{J}\left(\Psi_{1}+\Psi_{2}\right) .
\end{gathered}
$$

$\Psi_{1}$ provides the correction to rotations, while $\Psi_{2}$ provides the "pushed forward" constitutives stiffnesses. They are divided in this way for clarity during the derviation since they are arrived at using different methods. $J$ in equation (4.0.1) represents the Jacobian for the entire SM, as opposed to just the constituent of interest.

For constitutive calculations, collagen will have a "C" subscript (i.e. $\Psi_{1 \mathrm{C}}$ ), proteoglycan will have a "P" subscript (i.e. $\Psi_{1 \mathrm{P}}$ ), and the ground substance will have an "M" subscript (i.e. $\Psi_{1 \mathrm{M}}$ ). $\Psi_{1}$ requires the calculation of Cauchy stress, and this is done using the stress-strain relations derived in Chapter 3. $\Psi_{2}$ can be calculated using the following relationship defined in the reference configuration:

$$
C_{A B C D}=2 \frac{\partial S_{A B}}{\partial C_{C D}}
$$


Additionally, once the derivative shown in equation (4.0.2) is completed, the transformation shown in equation (4.0.3) can be used to achieve the stiffness in the deformed configuration:

$$
C_{i j k l}=F_{i A} F_{j B} F_{k C} F_{l D} C_{A B C D} J^{-1} .
$$

Stiffness contributions for the collagen, proteoglycan, and ground substance components are then calculated. These stiffnesses are combined to form the overall stiffness based on the stress balance hypothesis.

The derivation of the constitutive material stiffness matrix $\left(\widetilde{\mathbf{C}}^{\text {const }}\right)$ had previously utilized the constitutive deformation tensor $\left(\mathbf{C}^{\text {const }}\right)$ when calculating the partial derivative of Second Piola-Kirchhoff stress,

$$
\widetilde{\mathbf{C}}^{\text {const }}=2 \frac{\partial \mathbf{S}^{\text {const }}}{\partial \mathbf{C}^{\text {const }}}
$$

where "const" refers to the constituent of interest [3]. The derivative should actually be taken in terms of the overall deformation of the entire SM in order to reach the proper solution, as shown in equation (4.0.5) [37]:

$$
\widetilde{\mathbf{C}}^{\text {const }}=2 \frac{\partial \mathbf{S}^{\text {const }}}{\partial \mathbf{C}^{S M}}
$$

Utilizing these equations gives the exact form of the material stiffness matrix. 


\subsubsection{Ground Substance Stiffness $-\Psi_{1 \mathrm{M}}$}

To derive the $\Psi_{1 \mathrm{M}}$ component of the ground substance stress, the Cauchy stress term (T) must be calculated. Once this calculation is completed, the Cauchy stress can be inserted into the $\Psi_{1 \mathrm{M}}$ equation to define that term of the J-K ground substance stiffness contribution.

The ground substance stress equation, repeated here for convenience, is given in terms of the second Piola-Kirchhoff stress,

$$
\mathbf{S}^{M A T}=\mu\left(\mathbf{I}-\mathbf{C}^{-1}\right)
$$

Equation (3.2.2) can be transformed into Cauchy stress using equation (3.1.8), yielding the following:

$$
\mathbf{T}^{M A T}=\mu(\mathbf{B}-\mathbf{I}) J^{-1} .
$$

This is presented in terms of the Left Cauchy-Green tensor, B.

\subsubsection{Ground Substance Stiffness $-\Psi_{2 \mathrm{M}}$}

In indicial notation, the ground substance stress can be written as:

$$
S_{A B}^{M A T}=\mu\left(\delta_{A B}-C_{A B}^{-1}\right)
$$

The derivative shown in equation (4.0.5) of equation (4.1.2) results in

$$
\widetilde{C}_{A B C D}^{M A T}=\mu\left(C_{A C}^{-1} C_{B D}^{-1}+C_{A D}^{-1} C_{B C}^{-1}\right)
$$


which is a nontrivial result. The derivative is detailed in Appendix A. Once again note that this derivative was taken with respect to the overall deformation tensor $\left(\mathbf{C}^{S M}\right)$ as opposed to the constitutive deformation tensor $\left(\mathbf{C}^{\text {const }}\right)$. The transformation of equation (4.0.3) is then used to transform equation (4.1.3) into the deformed configuration:

$$
\widetilde{C}_{i j k l}^{M A T}=\mu\left(\delta_{i k}^{-1} \delta_{j l}^{-1}+\delta_{i l}^{-1} \delta_{j k}^{-1}\right) J^{-1}
$$

Combining equations (4.1.1) and (4.1.4) yields the total ground substance contribution to the stiffness matrix:

$$
\widetilde{C}_{i j k l}^{M A T}=J^{-1}\left[\begin{array}{l}
\frac{J}{2}\left[\delta_{l i} T_{k j}^{M A T}+\delta_{k j} T_{i l}^{M A T}+\delta_{k i} T_{l j}^{M A T}+\delta_{l j} T_{i k}^{M A T}\right]+ \\
\mu\left(\delta_{i k}^{-1} \delta_{j l}^{-1}+\delta_{i l}^{-1} \delta_{j k}^{-1}\right) J^{-1}
\end{array}\right] .
$$

\subsubsection{Collagen Stiffness $-\Psi_{1 \mathrm{C}}$}

The bimodular collagen stress can be determined using the linear stressstrain relationship found in equation (3.2.6), repeated here for convenience:

$$
\begin{aligned}
\mathbf{S}^{B I M}= & \gamma_{1}\left[\lambda_{1}\right]\left(\lambda_{1}^{2}-1\right) \mathbf{M}_{1}+\gamma_{2}\left[\lambda_{2}\right]\left(\lambda_{2}^{2}-1\right) \mathbf{M}_{2}+\gamma_{3}\left[\lambda_{3}\right]\left(\lambda_{3}^{2}-1\right) \mathbf{M}_{3} \\
& +\delta\left[\lambda_{ \pm 12}\right]\left(\lambda_{ \pm 12}^{2}-1\right) \mathbf{M}_{ \pm 12} \\
& +\delta\left[\lambda_{ \pm 13}\right]\left(\lambda_{ \pm 13}^{2}-1\right) \mathbf{M}_{ \pm 13} \\
& +\delta\left[\lambda_{ \pm 23}\right]\left(\lambda_{ \pm 23}^{2}-1\right) \mathbf{M}_{ \pm 23}
\end{aligned}
$$

The following derivation follows the work performed in [3]. Equation (3.2.6) contains nine terms that can be used to populate a three-by-three matrix. This is 
convenient because it allows for the use of summation notation when coding within the UMAT.

A fiber angle matrix can be constructed that accounts for fiber angles in the primary and secondary directions:

$$
\Phi=\left(\begin{array}{ccc}
\phi_{11} & \phi_{+12} & \phi_{+13} \\
\phi_{+12}+90^{\circ} & \phi_{22} & \phi_{+23} \\
\phi_{+13}+90^{\circ} & \phi_{+13}+90^{\circ} & \phi_{33}
\end{array}\right),
$$

where $\phi_{11}=\phi_{22}=\phi_{33}=0$. This definition can be used to modify the general structural array given in equation (3.2.5), to be in summation notation:

$$
\begin{aligned}
& M_{i j K L}= \\
& \sum_{i, j=1}^{3}\left\{\cos ^{2} \Phi_{i j} \delta_{i K} \delta_{i L}+\sin ^{2} \Phi_{i j} \delta_{j K} \delta_{j L}+\cos \Phi_{i j} \sin \Phi_{i j}\left(\delta_{i K} \delta_{j L}+\delta_{j K} \delta_{i L}\right)\right\}
\end{aligned}
$$

where each pair of indices $(K, L)$ is used to define one structural tensor, $M_{\mathrm{ijKL}}$. For instance, completing the summation over $\mathrm{i}, \mathrm{j}$ for a given $\mathrm{K}, \mathrm{L}$ would allow for the population of that particular $M$ tensor (i.e. $\Sigma M_{\mathrm{ij} 11}=\mathbf{M}_{11}$ and $\Sigma M_{\mathrm{ij} 12}=\mathbf{M}_{12}$ ).

Another matrix can also be constructed for convenience that contains the bimodular constants that control the "on-off" functionality:

$$
\Gamma=\left(\begin{array}{lll}
\gamma_{1}\left[\lambda_{1}\right] & \delta\left[\lambda_{+12}\right] & \delta\left[\lambda_{+13}\right] \\
\delta\left[\lambda_{-12}\right] & \gamma_{1}\left[\lambda_{1}\right] & \delta\left[\lambda_{+23}\right] \\
\delta\left[\lambda_{-13}\right] & \delta\left[\lambda_{-23}\right] & \gamma_{1}\left[\lambda_{1}\right]
\end{array}\right) .
$$


Additionally, a matrix of stretch squared terms can be expressed in general summation form as

$$
\Lambda_{i j}^{2}=\sum_{K, L=1}^{3} M_{i j K L} C_{K L},
$$

where $C_{K L}$ is the right Cauchy-Green deformation tensor. By introducing these tensors, the elastic bimodular collagen stress equation from equation (3.2.6) can be simplified to

$$
S_{A B}^{B I M}=\sum_{i, j=1}^{3} \Gamma_{i j}\left(\Lambda_{i j}^{2}-1\right) M_{i j A B} .
$$

The transformation to Cauchy stress found in equation (3.1.8) can then be applied to derive the bimodular Cauchy stress contribution:

$$
T_{k, l}^{B I M}=\sum_{i, j, A, B=1}^{3} \Gamma_{i j}\left(\Lambda_{i j}^{2}-1\right) M_{i j A B} F_{i A} F_{j B} J^{-1},
$$

where $J$ is the overall Jacobian, and the $F$ 's are overall deformation gradients. This is a change to the previous work where only the collagen constituent Jacobians and deformation gradients had been applied.

\subsubsection{Collagen Stiffness $-\Psi_{2 \mathrm{C}}$}

The same methodology utilized for $\Psi_{2 \mathrm{M}}$ is then applied to the linear bimodular stress relationship found in equation (4.1.9), to yield the bimodular contribution to the collagen stiffness of $\Psi_{2 \mathrm{C}}$ : 


$$
\widetilde{C}_{i j k l}^{b i m}=\sum_{m, n, A, B, C, D=1}^{3}\left\{\begin{array}{l}
\Gamma_{m n}\left(\Lambda^{2}{ }_{m n}\right) M_{m n A B} M_{m n C D} \\
\left(F_{k C} F_{l D}+F_{k D} F_{l C}\right) F_{i A} F_{j B}
\end{array}\right\} J^{-1} .
$$

The combination of the bimodular portions of $\Psi_{1 \mathrm{C}}$ and $\Psi_{2 \mathrm{C}}$ yield the final collagen contribution to the material stiffness matrix for the J-K Method:

$$
\widetilde{C}_{i j k l}^{C O L}=J^{-1}\left[\left(\begin{array}{l}
\frac{J}{2}\left[\delta_{l i} T_{k j}^{C O L}+\delta_{k j} T_{i l}^{C O L}+\delta_{k i} T_{l j}^{C O L}+\delta_{l j} T_{i k}^{C O L}\right]+ \\
\left.\sum_{\substack{m, n, A B, C, D=1}}^{3}\left\{\begin{array}{l}
\Gamma_{m n}\left(\Lambda_{m n}^{2}\right) M_{m n A B} M_{m n C D} \\
\left(F_{k C} F_{l D}+F_{k D} F_{l C}\right) F_{i A} F_{j B}
\end{array}\right\}\right)
\end{array}\right] .\right.
$$

\subsubsection{Proteoglycan Stiffness $-\Psi_{1 \mathrm{P}}$}

$\Psi_{1 \mathrm{P}}$ requires the Cauchy stress calculation for the proteoglycan constituent. Utilizing the transformation in equation (3.1.8), the proteoglycan second Piola-Kirchhoff stress equation (equation (3.2.8)) can be converted to Cauchy stress:

$$
\mathbf{T}^{P G}=\frac{-\alpha_{1}^{*}}{\left(J^{P G}\right)^{\alpha_{2}}} * \mathbf{I}=\frac{-\left(\alpha_{1}\left(\rho_{O}^{P G}\right)^{\alpha_{2}}\right.}{\left(\operatorname{det}\left(\mathbf{C}^{P G}\right)\right)^{\alpha_{2}}} * \mathbf{I} .
$$

This Cauchy stress equation populates the $T$ values found within the $\Psi_{1 \mathrm{P}}$ term. 


\subsubsection{Proteoglycan Stiffness $-\Psi_{2 \mathrm{P}}$}

The $\Psi_{2 \mathrm{P}}$ requires the use of the derivation technique used for collagen. The proteoglycan second Piola-Kirchhoff stress (equation (3.2.8)) is derived using equation (4.0.5). Using the chain rule, the $\Psi_{2 \mathrm{P}}$ can be expanded to

$$
\Psi_{2 P}=2 F_{i A} F_{j B} F_{k C} F_{I D}\left[\begin{array}{l}
\left(\frac{-\alpha_{1}^{*}}{\left(\operatorname{det} C^{P G}\right)^{\frac{1}{2}\left(\alpha_{2}-1\right)}}\right)\left(\frac{\partial C_{A B}^{-1}}{\partial C_{C D}^{-1}}\right) \\
+\frac{\partial}{\partial C_{C D}}\left(\frac{-\alpha_{1}^{*}\left(-\frac{1}{2}\left(\alpha_{2}-1\right)\right)}{\left(\operatorname{det} C^{P G}\right)^{\frac{1}{2}\left(\alpha_{2}+1\right)}}\right) C_{A B}^{-1}
\end{array}\right],
$$

where the derivative of the determinant can be defined as [33]:

$$
\frac{\partial}{\partial C_{C D}}\left(\operatorname{det} C^{P G}\right)=\left[\operatorname{det}\left(F_{O}^{P G}\right)\right]^{2} I_{3}\left(C_{D C}^{-1}\right) .
$$

Using the identity of a partial derivative a tensor with respect to its inverse as defined in Appendix A, a final value for $\Psi_{2 \mathrm{P}}$ in the reference configuration is as follows:

$$
\Psi_{2 P}=2 F_{i A} F_{j B} F_{k C} F_{I D}\left[\begin{array}{l}
\left(\frac{-\alpha_{1}^{*}}{\left(\operatorname{det} C^{P G}\right)^{\frac{1}{2}\left(\alpha_{2}-1\right)}}\right)\left(-\frac{1}{2}\left[C_{A C}^{-1} C_{B D}^{-1}+C_{A D}^{-1} C_{B C}^{-1}\right]\right) \\
+\left(\frac{-\alpha_{1}^{*}\left(\frac{1}{2}\left(1-\alpha_{2}\right)\right.}{\left(\operatorname{det} C^{P G}\right)^{\frac{1}{2}\left(\alpha_{2}+1\right)}}\right)\left[\operatorname{det}\left(F_{O}^{P G}\right)\right]^{2} I_{3} C_{D C}^{-1} C_{A B}^{-1}
\end{array}\right] J^{-1}
$$


Using the transformation from the reference configuration to the deformed configuration in equation (4.0.3), $\Psi_{2 \mathrm{P}}$ becomes

$$
\Psi_{2 P}=\frac{2}{J} *\left[\begin{array}{c}
\left(\frac{\alpha_{1}^{*}}{\left(J^{P G}\right)^{\alpha_{2}-1}}\right)\left(\frac{1}{2}\left[\delta_{i k} \delta_{j l}+\delta_{i l} \delta_{j k}\right]\right)+ \\
\left.\left(\frac{-\alpha_{1}^{*}\left(\frac{1}{2}\left(1-\alpha_{2}\right)\right)}{\left(J^{P G}\right)^{\alpha_{2}+1}}\right)\left[\operatorname{det}\left(F_{O}^{P G}\right)\right]^{2} I_{3} \delta_{l k} \delta_{i j}\right]
\end{array}\right]
$$

in the deformed configuration. Putting together $\Psi_{1 \mathrm{P}}$ and $\Psi_{2 \mathrm{P}}$ yields the total proteoglycan contribution to the material stiffness matrix for the J-K method:

$$
\widetilde{C}_{i j k l}^{P G}=\left\{\begin{array}{c}
\frac{1}{2}\left[\delta_{l i} T_{k j}^{P G}+\delta_{k j} T_{i l}^{P G}+\delta_{k i} T_{l j}^{P G}+\delta_{l j} T_{i k}^{P G}\right]+ \\
\left.\left[\begin{array}{c}
\left(\frac{\alpha_{1}^{*}}{\left(J^{P G}\right)^{\alpha_{2}-1}}\right)\left(\frac{1}{2}\left[\delta_{i k} \delta_{j l}+\delta_{i l} \delta_{j k}\right]\right)+ \\
\frac{2}{J^{2}} *\left[\frac{-\alpha_{1}^{*}\left(\frac{1}{2}\left(1-\alpha_{2}\right)\right)}{\left(J^{P G}\right)^{\alpha_{2}+1}}\right)
\end{array}\right]\left[\operatorname{det}\left(F_{O}^{P G}\right)\right]^{2} I_{3} \delta_{l k} \delta_{i j}\right]
\end{array}\right\} .
$$

The combination of equations (4.1.19), (4.1.13), and (4.1.5) leads to the overall material stiffness matrix, including proteoglycan, collagen, and ground substance constituents, respectively. 


\subsection{Implementation and Verification}

\subsubsection{Convergence Study}

Once the J-K Method was implemented into the UMAT code, a convergence study was performed to determine the amount of elements necessary to accurately approximate the results. Uniaxial tension (UT), confined compression (CC), and unconfined compression (UCC) experimental protocols were performed. Simulations were executed for $1,27,64$, and 343 elements composing a 2 millimeter cube for both elastic and poroelastic models. All studies were done at experimental strain levels: $0 \%, 15 \%, 30 \%$, and $45 \%$ strains for UCC / CC, and $0 \%$ and $10 \%$ strains for UT.

The elastic model yielded identical stresses between the different element number simulations. This means that the model can be considered to be accurate with just a 1 element elastic model.

Poroelasticity has been previously implemented into the PFD method, but had been left out up until this point for simplicity. Poroelasticity was added to the $\mathrm{J}-\mathrm{K}$ method prior to running the poroelastic convergence, utilizing the theory previously discussed. The poroelastic convergence study showed that increasing the number of elements does not significantly impact the results. The maximum difference between the 1 and 343 element models was $0.09 \%$ for the $45 \%$ strain CC 
test. This is small relative to the stresses that are being outputted, meaning that the 1 element model accurately approximates the results. The convergence study plots are shown in Appendix B.

It should be noted that minor stress discontinuities were found between the faces defined as having the zero pore pressure boundary condition, and the rest of the model. This is shown in Figure 4.1.

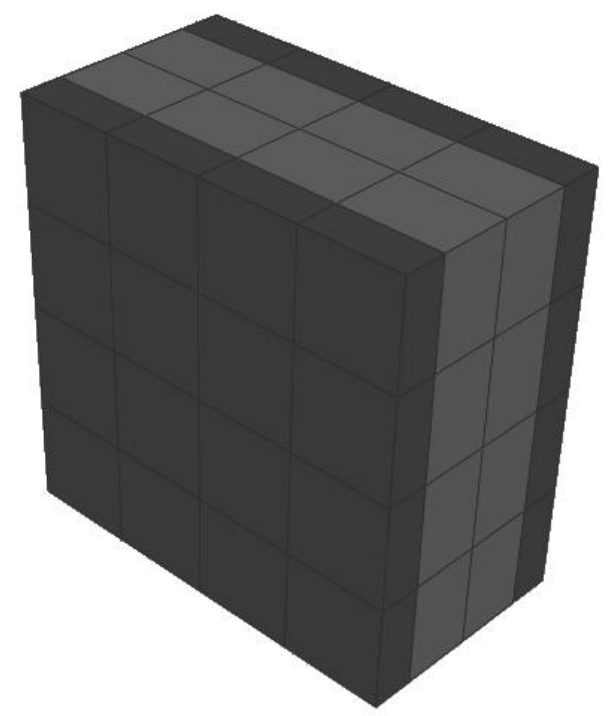

Figure 4.1: 64 element cubic poroelastic model under 45\% confined compression. The stress discontinuities are shown as the color change between elements.

Discontinuities will sometimes occur in the poroelastic analyses due to a gradient in pore pressure. When a load or deformation is applied to the surface, as is done in $\mathrm{CC}$, the fluid will initially bear the majority of the load and then gradually be transferred to the SM. This discontinuity in pressure directly correlates with a discontinuity in pressure, and is a behavior inherent to the poroelastic model. The 
difference in stresses between the edge and body elements are magnitudes lower than the overall stress, so they do not impact the results of this convergence study. The stresses shown for the body still accurately represent the overall stress state.

\subsubsection{Validation Using Previous Models}

Previous work implemented PFD and DPF protocols, as already discussed [3]. The PFD protocol was used as a check for the J-K method implementation. The J-K and PFD methods were tested at identical strains to ensure they output the same stresses. The PFD code had been optimized to match the experimental results, as is explained in Chapter 5. This provided a baseline simulation that matched the experimental simulations; thus, if the J-K method could match the PFD simulations, then it is assumed that the J-K method can accurately simulate experimental results. The overall stress equations did not change significantly between the three methods, meaning that all stress outputs should be similar for the three experimental protocols. The UMAT file contains all of the changes to the material Jacobian matrix that were discussed in the derivation section. These UMATs are contained in Appendix E for reference.

The goal of this experiment was to modify the way ABAQUS calculated the stiffness. Due to this, the way the material parameters defined the AC behavior remained the same for all stiffness matrix types. This allowed for the material parameters being sent into the UMAT to remain constant for DPF, PFD, and J-K 
methods. These parameters are summarized in Table 4.1. The input file contained a single C3D8 type element that was used for all tests. This file is included in Appendix E for reference. These tests only utilized the elastic portion of the input file, as opposed to using the poroelastic features. This was done to simplify the calculations being performed by ABAQUS, as well as lower computation time. Step by step calculations were outputted to the ".msg" file using the ABAQUS "WRITE" function within the UMAT. This allowed for checking the stress, strain, and stiffness outputs on a constitutive basis as ABAQUS stepped through its iterations. Note that all simulations were performed using ABAQUS version 6.7-3 on a Dell Latitude D620 laptop running Microsoft Windows XP Professional Edition.

To verify the J-K method code implementation within the UMAT, UT, CC, and UCC experimental protocols were tested using all three stiffness methods. CC and UCC tests were taken to $0 \%, 15 \%, 30 \%$, and $45 \%$ nominal strain levels in the $\mathbf{E}_{\mathbf{3}}$ material direction, per experimental protocols discussed in Chapter 2. The UT test was performed at $0 \%$ and $10 \%$ nominal strain levels in the $\mathbf{E}_{\mathbf{2}}$ material direction. The $\mathrm{CC}$ test had boundary conditions of zero displacement along lateral faces $( \pm 1, \pm 2)$, while the UT and UCC tests allowed for lateral expansion on their lateral faces: $\pm 1, \pm 3$ and $\pm 1, \pm 2$, respectively. Cauchy stresses and Poisson's ratios were measured for each strain level and the three stiffness 
methods were compared to one another. This ensures that the basic stress laws are correctly implemented in the J-K method.

After running the simulations, there was no difference in Cauchy stress levels between the three methods in all three test cases. This leads to the conclusion that the stress-strain relations were implemented correctly between the methods and the J-K method can accurately simulate the experimental results.

\subsection{Performance}

Once the models were validated for stress-strain relations, the performance of the new material stiffness was evaluated using a maximum strain criterion. This criterion tests the capability of the model to effectively execute problems with large deformations.

\subsubsection{Methods}

This test utilized the CC, UCC, and UT experiments explained previously. For each experimental protocol, the same material parameters as shown in Tables 4.1 and 4.2 were used. An elastic analysis was performed as before. Additionally, each of the tests consisted of three distinct analysis steps. The initial step was a stress equilibrium determining step. Following this, a ramp to the prescribed displacement was applied statically. The third step re-established equilibrium, by holding the model at the displacement prescribed in step two. 
Table 4.1: Collagen equation parameters used while verifying J-K method subroutine, and testing all subroutines for maximum convergence.

\begin{tabular}{|c|c|c|c|c|c|c|c|}
\hline $\begin{array}{c}\mu \\
{[\mathrm{MPa}]}\end{array}$ & $\begin{array}{c}\gamma_{1} \\
{[\mathrm{MPa}]}\end{array}$ & $\begin{array}{c}\gamma_{2} \\
{[\mathrm{MPa}]}\end{array}$ & $\begin{array}{c}\gamma_{3} \\
{[\mathrm{MPa}]}\end{array}$ & $\begin{array}{c}\delta \\
{[\mathrm{MPa}]}\end{array}$ & $\begin{array}{c}\phi_{12} \\
{[\mathrm{rad}]}\end{array}$ & $\begin{array}{c}\phi_{13} \\
{[\mathrm{rad}]}\end{array}$ & $\begin{array}{c}\phi_{23} \\
{[\mathrm{rad}]}\end{array}$ \\
\hline 0.032 & 1.38 & 1.38 & 0.69 & 1.38 & 0.785 & 0.611 & 0.611 \\
\hline
\end{tabular}

Table 4.2: Proteoglycan equation parameters used while verifying J-K method subroutine, and testing all subroutines for maximum convergence.

\begin{tabular}{|c|c|c|c|}
\hline $\begin{array}{c}\alpha_{1} \\
{[-]}\end{array}$ & $\begin{array}{c}\alpha_{2} \\
{[-]}\end{array}$ & $\begin{array}{c}\alpha_{1}^{*} \\
{[\mathrm{MPa}]}\end{array}$ & $\begin{array}{c}\rho_{O}^{P G} \\
{\left[\mathrm{~g} / \mathrm{cm}^{3}\right]}\end{array}$ \\
\hline 85.9 & 2.54 & 0.02579 & 0.04104 \\
\hline
\end{tabular}

\subsubsection{Results}

The maximum strains that could be applied for each test condition, while still yielding convergence are plotted in Figure 4.1 according to UMAT used. For UT, the J-K and DPF methods converged to $3700 \%$ strain, while the PFD method converged to $663 \%$ strain. For UCC, the J-K method converged to $84.5 \%$ strain, DPF converged to $82.5 \%$ strain, and PFD converged to $80.0 \%$ strain. All methods converged to $99 \%$ strain for CC. 


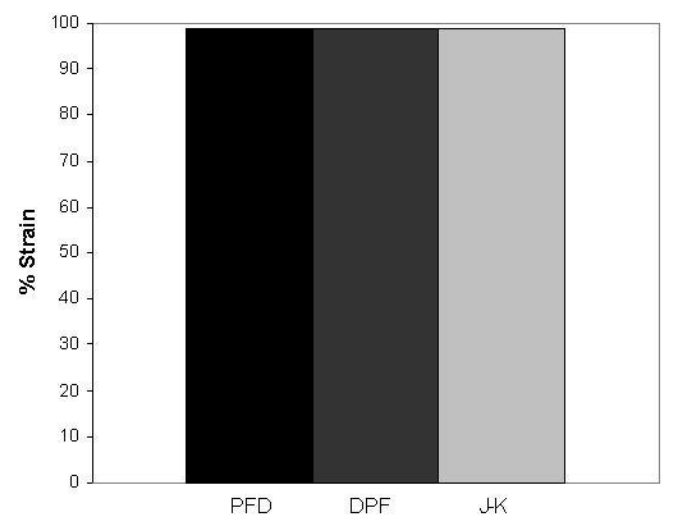

cc

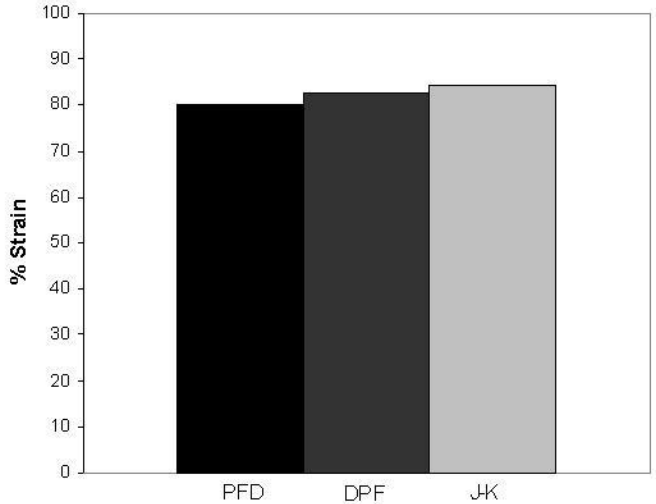

ucc

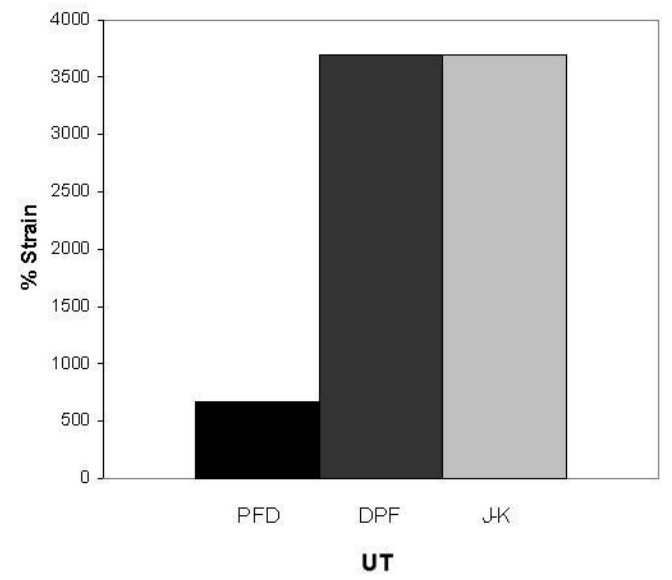

Figure 4.2: Maximum convergence strains for PFD, DPF, and J-K simulations. Data is shown for uniaxial tension (UT), confined compression (CC), and unconfined compression (UCC).

\subsubsection{Conclusions}

The J-K method showed the ability to converge to higher strains than the previously implemented methods. This was more apparent in tests that allowed for more free boundary conditions. The CC test in particular constrains many of the degrees of freedom, which results in a simple solution. For this reason, all 
model reached the same convergence for this test. For UCC, it was expected that the DPF and J-K methods would converge to the same strains. The J-K method was able to achieve higher strains. The J-K method does have terms that are present in the stiffness matrix regardless of the presence of rotations in the simulated test. These terms would affect the convergence regardless of rotations.

It is recommended that further use of cartilage mechanical model utilize the J-K method for material stiffness calculation. The correction terms $\left(\Psi_{1}\right)$ are used to correct for possible rotations in the deformed configuration, making it more applicable to a variety of loading conditions. The stress-strain relation remained the same for all analyses, meaning that any material parameter determinations can use any of the three material stiffness models, as long as they are within the convergence range. 


\section{Chapter 5}

\section{Quantifying Material Parameters}

One of the major goals of this work was to accurately quantify the material parameters based on experimental data. The experimental data was gathered using the UT, UCC, and CC protocols previously discussed. The AC samples were all taken from newborn bovine (1-3 weeks old) knees, specifically the patellofemoral groove. The mechanical and biochemical properties of the UT and UCC samples were based on the work completed in [4]. CC mechanical and biochemical properties were based on the permeation study day zero groups (P00) completed internally by the CPCBG

The PFD model was used to run all material simulations. The PFD is not significantly different from the DPF or J-K methods in the way that it utilizes the material parameters. For this reason, all three models will give the same stress response for the same material parameters. 


\subsection{Elastic Material Constant Overview}

The majority of the elastic material constants utilized in this work have been defined in Chapter 3. They are summarized in Table 5.1 for convenience.

Table 5.1: Elastic constitutive law material constants.

\begin{tabular}{|c|c|c|c|c|}
\hline $\begin{array}{c}\text { Con- } \\
\text { stituent }\end{array}$ & $\begin{array}{c}\text { Sym- } \\
\text { bol }\end{array}$ & Description & Equation [Units] & Source \\
\hline \multirow{4}{*}{ PG } & $\overline{\alpha_{1}}$ & $\begin{array}{l}\text { Isotropic curve fit } \\
\text { coefficient }\end{array}$ & - & [14] \\
\hline & $\alpha_{2}$ & $\begin{array}{l}\text { Isotropic curve fit } \\
\text { exponent }\end{array}$ &,$-[-]$ & [14] \\
\hline & $\alpha_{1}^{*}$ & \begin{tabular}{|l} 
Initial PG stress \\
\end{tabular} & $\begin{array}{c}\alpha_{1}^{*}=\alpha_{1}\left(\rho_{o}^{P G}\right)^{\alpha_{2}}, \\
{[\mathrm{MPa}]}\end{array}$ & Calculated \\
\hline & $\rho_{O}^{P G}$ & $\begin{array}{l}\text { Initial PG appar- } \\
\text { ent density }\end{array}$ & $\begin{array}{c}\rho_{O}^{P G}=\frac{\text { mass }_{P G}}{\text { wet weight }}, \\
{\left[\mathrm{g} / \mathrm{cm}^{3}\right]}\end{array}$ & $\begin{array}{l}\text { Unpublished data } \\
\text { (P00 samples) }\end{array}$ \\
\hline \multirow{7}{*}{$\mathrm{COL}$} & $\gamma_{1}$ & $\begin{array}{l}\text { Tensile coefficient } \\
\text { for direction } \mathbf{E}_{1}\end{array}$ &,- , [MPa] & $\begin{array}{c}\text { Calculated from data } \\
{[4,20]}\end{array}$ \\
\hline & $\gamma_{2}$ & $\begin{array}{l}\text { Tensile coefficient } \\
\text { for direction } \mathbf{E}_{2}\end{array}$ & $\gamma_{2}=\gamma_{1},[\mathrm{MPa}]$ & $\begin{array}{c}\text { Calculated from data } \\
{[4,20]}\end{array}$ \\
\hline & $\gamma_{3}$ & $\begin{array}{l}\text { Tensile coefficient } \\
\text { for direction } \mathbf{E}_{3}\end{array}$ & $\gamma_{3}=\gamma_{1} / 2,[\mathrm{MPa}]$ & $\begin{array}{c}\text { Calculated from data } \\
{[4,20]}\end{array}$ \\
\hline & $\delta$ & $\begin{array}{l}\text { Tensile coefficient } \\
\text { for directions } \mathbf{E}_{ \pm 12}, \\
\mathbf{E}_{ \pm 13}, \mathbf{E}_{ \pm 23}\end{array}$ & $\delta=\gamma_{1},[\mathrm{MPa}]$ & $\begin{array}{c}\text { Calculated from data } \\
{[4,20]}\end{array}$ \\
\hline & $\phi_{12}$ & $\begin{array}{l}\text { Angle defining } \\
\text { direction } \mathbf{E}_{ \pm 12}\end{array}$ & 45, [deg] & [20] \\
\hline & $\phi_{13}$ & $\begin{array}{l}\text { Angle defining } \\
\text { direction } \mathbf{E}_{ \pm 13}\end{array}$ & $35,[\mathrm{deg}]$ & [20] \\
\hline & $\phi_{23}$ & $\begin{array}{l}\text { Angle defining } \\
\text { direction } \mathbf{E}_{ \pm 23}\end{array}$ & $35,[\mathrm{deg}]$ & [20] \\
\hline$\overline{\mathrm{SM}}$ & $\bar{\mu}$ & $\begin{array}{l}\text { Infinitesimal shear } \\
\text { modulus }\end{array}$ &,$-[\mathrm{MPa}]$ & $\begin{array}{l}\text { Unpublished data } \\
\text { (P00 samples) }\end{array}$ \\
\hline
\end{tabular}




\subsection{Collagen Constants}

The $\phi$ terms are taken from previous studies, where they were determined by varying fiber angle and fitting the results to measured typical adult bovine $\mathrm{AC}$ mechanical properties [20] but also allow for typical anisotropy measured for newborn bovine AC [19]. As stated, $\phi$ terms represents the principle and secondary collagen fiber directions. The elastic response in UT is primarily dependent upon the material parameters $\gamma_{1}, \gamma_{2}, \gamma_{3}$, and $\delta$. The experimental protocols only gathered tensile information in the $\mathbf{E}_{2}$ direction, which means that the remaining directional properties needed to be approximated. A simplified constraint of $\gamma_{1}=$ $\gamma_{2}=2 \gamma_{3}=\delta$ is used for this work, which gives approximately equal tensile modulus in the 1 and 2 directions. This has been shown experimentally in [13] and yielded reasonable mechanical properties in simulation [19].

To calculate the $\gamma_{1}$ parameter, a Matlab code was used that simultaneously solves for the initial collagen deformation gradient tensor, $\mathbf{F}_{o}^{C O L}$, and $\gamma_{1}$ based on the proteoglycan alpha constants as well as equilibrium tensile stress at $10 \%$ strain [21]. The $\gamma$ values were initially checked for proper implementation using a zero stress configuration simulation in ABAQUS. $\mathbf{F}_{o}^{C O L}$ and $\mathbf{F}_{o}^{P G}$ were set equal to $\mathbf{I}$, while proteoglycan contributing terms, $\alpha_{1}$ and $\alpha_{2}$, were set to zero. $\gamma$ were generated based on experimental data values from $[22,23]$. A zero strain condition was imposed on the tensile block, leading, which resulted in zero stress in the refer- 
ence configuration. A custom Matlab code which runs a tensile experiment, was also used to verify these results. This code was developed in [21]. This suggests that the material properties were interacting as expected and simulations could move forward.

When all experimental parameters were implemented, ABAQUS was unable to reach a zero stress configuration using the values directly from the Matlab code used previously mentioned that simultaneously solves for material values [21]. As discussed, $\mathbf{F}_{o}^{C O L}$ prescribes an initial deformation gradient that counteracts the swelling created by the proteoglycan component. It is critical that the ABAQUS simulation begin with a stress free configuration so that the results are not skewed when strains greater than zero are applied. This led to the iteration of $\mathbf{F}_{o}^{C O L}$ initial conditions using ABAQUS.

An initial $\mathbf{F}_{o}^{C O L}=\mathbf{I}$ was used, which led to deformation in the "reference configuration" due to unbalanced proteoglycan and collagen stress components. An iterative calculation detailed in Appendix $\mathrm{C}$ was used to approximate the initial $\mathbf{F}_{o}^{C O L}$ to a value greater than $\mathbf{I}$ that allowed for a stress free reference configuration. It should be noted that the initial $\mathbf{F}_{o}^{C O L}$ needs to be adjusted whenever a material constituent parameter is modified. For this reason, the initial $\mathbf{F}_{o}^{C O L}$ is updated on a simulation by simulation basis to optimize individual parameters. This 
is done until all parameters have been determined, and then a true $\mathbf{F}_{o}^{C O L}$ is solved for.

A $\gamma_{1}$ optimization study was performed that varied $\gamma_{1}$ from 0.25 to 1.50 to determine the effect on compressive and tensile test results. The resulting graphs are shown in Appendix D. As expected, there was a large effect on the tensile results when manipulating $\gamma_{1}$, and a small effect on the compressive results. Additionally, the UCC Poisson's ratios showed an inverse relationship to modulus. As $\gamma_{1}$ increased, the modulus in compression increased, but a decrease in Poisson's ratios. The Poisson's ratios were greatly affected by the changes to $\gamma_{1}$ values.

From there, $\gamma_{1}$ was initially assigned values based on the equilibrium tensile modulus data from [11]. This data has been utilized in the poroviscoelastic model developed in [21]. The tensile modulus in the experiments by [11] stretched the cartilage samples to $10 \%$ strain and then allowed them to relax for $900 \mathrm{~s}$ before recording the equilibrium stress. Simulations were performed using the corresponding $\gamma$ values that yielded the proper tensile stresses from [11]; however, it was noted that the behavior in compression yielded higher than expected aggregate moduli in CC and lower than expected Poisson's ratios in UCC.

As mentioned in Section 2.2.3, it was hypothesized that the tensile experiments performed in [11] had not reached a completely relaxed state after the 
$900 \mathrm{~s}$ relaxation period. For this reason, the data collected by CPCBG utilized a 5000 s relaxation time curve fit [4]. This led to a lowered $\gamma_{1}$ that more accurately simulated the fully relaxed cartilage samples in tension.

\subsection{Proteoglycan Constants}

The proteoglycan constituent is based on the Modified Donnan (MD) model for AC developed in [14], that predicts swelling pressures based on experimental parameters [39]. Previous work has utilized the extended two compartmental swelling stress model [21], but simulations run in this work showed that the MD model more accurately represented the behavior of AC in compression. See Appendix D for comparison plots of the two methods.

The $\rho_{O}^{P G}$ component of the proteoglycan constituent was calculated using experimental mass and wet weight measurements (see equation found in Table 5.1). The necessary proteoglycan weights and sample wet weights were taken

from biochemistry data of permeation day zero (P00) samples. Utilizing $\rho_{O}^{P G}$ and the alpha constants, equation (5.3.1) can be used to calculate $\alpha_{1}^{*}$, which represents the proteoglycan stress in the reference configuration.

$$
\alpha_{1}^{*}=\alpha_{1}\left(\rho_{O}^{P G}\right)^{\alpha_{2}}
$$


Initially, $\alpha_{1}^{*}$ and $\alpha_{2}$ values were taken from the zero proteoglycan depletion groups found in [21]. These values yielded compressive moduli that were above experimental values, as well as UCC Poisson's ratios $(v)$ that were lower than the experimental values by multiple magnitudes. The difference between experimental and simulated results led to validation of the compressive tests.

A linearization study was conducted to verify the results being obtained in ABAQUS. An ABAQUS mechanical simulation was performed that set all collagen parameters $\left(\gamma_{1}, \gamma_{2}, 2 \gamma_{3}, \delta\right)$ to zero, essentially eliminating the collagen contribution. The SM and proteoglycan terms were nonzero, utilizing values from the MD model [14]. A UCC simulation was run to $30 \%$ strain, yielding a $v=0.30$. This showed that the lateral expansion was able to occur on the anticipated scale. A hand calculated linearization was also conducted (shown in Appendix A), to check the ABAQUS results. The hand calculation yielded $v=0.347$, which is close to the simulated value. This shows that the lateral expansion properties were behaving within the expected range.

$\alpha_{2}$ was then optimized over a range of values for UT, UCC, and CC experimental protocols. In order to modify the $\alpha_{2}$ parameter individually, a new ABAQUS input of $\alpha_{1}^{*}$ was created. This allowed for modification of the Cauchy stress equation as shown (written in terms of PFD UMAT code parameters): 


$$
\text { STRESS }=\alpha_{1}\left(\frac{\rho_{O}^{P G}}{\left(\operatorname{det} \mathbf{F}_{P}\right)}\right)^{\alpha_{2}} \mathbf{I} \rightarrow \text { STRESS }=\frac{-\alpha_{1}^{*}}{\left(\operatorname{det} \mathbf{F}_{P}\right)^{\alpha_{2}}} \mathbf{I}
$$

and similar modification to the stiffness equation :

$$
\begin{gathered}
\text { STIFFP }=\frac{-\alpha_{1}\left(\rho_{O}^{P G}\right)^{\alpha_{2}}\left(1-\alpha_{2}\right)}{\left(\operatorname{det} \mathbf{V}_{P}\right)^{\alpha_{2}}}(\mathbf{I}) \mathbf{V}_{P}^{-1} \\
\downarrow \\
\text { STIFFP }=\frac{-\alpha_{1}^{*}\left(1-\alpha_{2}\right)}{\left(\operatorname{det} \mathbf{V}_{P}\right)^{\alpha_{2}}}(\mathbf{I}) \mathbf{V}_{P}^{-1}
\end{gathered}
$$

By doing this, when $\alpha_{2}$ is modified, the initial proteoglycan stress, $\alpha_{1}^{*}$, remains unchanged. Note that $\alpha_{1}^{*}$ must be determined from the MD model to correspond to the initial GAG stress. This is based solely on the GAG density. Modifications to $\alpha_{2}$ then yield different GAG stiffnesses at different strains without altering the initial GAG stress. $\alpha_{2}$ was modified within a range of 2.30 and 2.80 to determine an optimal number. There was not a significant change in modulus or Poisson's ratio during compression over this range. This led to the conclusion that theoretically derived $\alpha_{1}$ and $\alpha_{2}$ MD parameters [14] combined with the experimental $\rho_{O}^{P G}$ data. would yield results close to experimental. It should also be noted that as $\alpha_{2}$ increased, the compressive modulus increased as well as the Poisson's ratio in UCC. 


\subsection{Solid Matrix Constants}

During the proteoglycan parameter optimization, values from the M1-D0 (superficial layer day zero AC) simulations were implemented [19]. In these simulations, the modulus decreased in compression as expected, but was still higher than that determined experimentally. The UCC Poisson's ratios were also low when compared to the experimental values. It was noted that $\mu=0$ in the data presented, whereas all ABAQUS simulations up to that point had used $\mu=$ 0.11. The $\mu=0.11$ value was taken from experimental torsion values [29]. In previous studies, it represented the infinitesimal shear modulus for collagen [3].

$\mu$ was then varied between 0.01 and 0.10 for ABAQUS simulations. The modification of the $\mu$ parameter had a significant impact on both the tensile and compressive moduli (shown in Appendix D), but little impact on the UCC Poisson's ratio. As $\mu$ decreased, the moduli both showed a downward trend. During these simulations, it was noted that with $\mu \approx 0$, the proteoglycan stresses were predicted to be less than the total CC stress.

The osmotic pressure of the proteoglycan component has been traditionally thought to capture the majority of the compressive properties of $\mathrm{AC}$ [40]. Recent studies have shown that the other (GAG) related molecular energies may contribute to the proteoglycan compressive resistance $[9,31]$. The results of the ABAQUS simulations support the idea of other forces contributing to the com- 
pressive stress, aside from the osmotic swelling pressure. Previous studies by Buschmann hypothesized that these GAG related energies are results of soluble ions interacting with the stationary proteoglycan macromolecules [31]. The osmotic swelling pressure in addition to these energetic responses provide a possible mechanism for defining the compressive properties of AC. The collagen component has also been shown to contribute to the compressive properties [13, 29].

The shear response of cartilage has been hypothesized to be largely governed by the tensile response of the collagen fibrils. Additionally, the complex distribution of the collagen fibers, especially in the middle zone, allows them to be pulled into tension when compressive forces are applied, and the SM is put into shear [41]. This allows cartilage to behave as expected when applying fiber reinforced composites theory [41]. Additionally, a positive correlation between shear stiffness and increases in compressive strain has been shown [41].

Previously, the $\mu$ term solely accounted for the infinitesimal shear properties due to collagen. Based on the simulations and previous research, $\mu$ has been redefined as a SM term $\left(\mathbf{S}^{M A T}\right)$ instead of just a collagen term. This allows $\mu$ to account for quantitatively unknown contributions from GAG, collagen, and other SM components. Due to this, the $\mu$ term depends on the entire SM deformation gradient, $\mathbf{F}$, as opposed to the collagen deformation gradient, $\mathbf{F}^{C O L}$. The J-K method has implemented this change, whereas the PFD method did not. The PFD 
simulations are still considered to be valid, because $\mathbf{F}^{\mathrm{COL}} \approx \mathbf{I}$, which leads to extremely small changes in the final stress outputs.

Since the $\mu$ term had a large effect on the tensile and compressive properties, this value was modified substantially from previous research to match the experimental results. An error analysis was performed for $\mathrm{CC}$ and $\mathrm{UT}$ in order to find the optimal $\mu$ value. The UT test was optimized to $10 \%$ strain and CC test was optimized to $30 \%$ strain. The UCC analysis was not included in this optimization study due to limitations in the code that made it difficult to approximate both moduli and Poisson's ratios. The UCC would also serve as a final validation step to determine the transfer of the material parameters to a different experimental protocol.

\subsection{Results}

The final values for all material parameters are summarized in Table 5.2. 
Table 5.2: Elastic constitutive law material constants resulting from parameter optimization studies.

\begin{tabular}{|c|c|c|}
\hline Constituent & Symbol & Value [Units] \\
\hline \multirow{4}{*}{ PG } & $\alpha_{1}$ & $85.9[\mathrm{MPa}]$ \\
\cline { 2 - 3 } & $\alpha_{2}$ & $2.54[-]$ \\
\cline { 2 - 3 } & $\alpha_{1}^{*}$ & 0.0257895 \\
\cline { 2 - 3 } & $\rho_{O}^{P G}$ & $0.0410364\left[\mathrm{~g} / \mathrm{cm}^{3}\right]$ \\
\hline \multirow{5}{*}{ COL } & $\gamma_{1}$ & $1.38307[\mathrm{MPa}]$ \\
\cline { 2 - 3 } & $\gamma_{2}$ & $1.38307[\mathrm{MPa}]$ \\
\cline { 2 - 3 } & $\gamma_{3}$ & $0.691535[\mathrm{MPa}]$ \\
\cline { 2 - 3 } & $\delta$ & $1.38307[\mathrm{MPa}]$ \\
\cline { 2 - 3 } & $\phi_{12}$ & $45[\mathrm{deg}]$ \\
\cline { 2 - 3 } & $\phi_{13}$ & $35[\mathrm{deg}]$ \\
\cline { 2 - 3 } & $\phi_{23}$ & $35[\mathrm{deg}]$ \\
\hline SM & $\mu$ & $0.032[\mathrm{MPa}]$ \\
\hline
\end{tabular}

All values were calculated as discussed previously in Chapter 5. $\alpha_{1}$ and $\alpha_{2}$ values are taken from the MD model developed by Oungoulian [14]. These values, combined with $\rho_{O}^{P G}$ taken from P00 biochemistry results, allow for the calculation of $\alpha_{1}^{*}$ using the equation found in Table 5.1. $\gamma$ and $\mu$ values were optimized to experimental data as already discussed. 


\section{Chapter 6}

\section{Results}

One of the primary aims of this work was to accurately define elastic material parameters to model day zero (D00) mechanical properties in UT, UCC, and CC. This chapter compares the simulated results for the new elastic material pa-

rameters developed in Chapter 5 to the experimental results obtained. Results are compared using all three stiffness methods: DPF, PFD, and J-K.

\subsection{Day Zero Mechanical Simulations}

Mechanical parameters were optimized to CC 30\% strains and UT 10\% strains as previously discussed. The error results for all strain levels for UCC, CC, and UT are summarized in Table 6.1. The raw simulated and experimental numbers have been included in Appendix D. 
Table 6.1: Summary of experimental error between ABAQUS simulations and experimental results. Results are presented for all three material stiffness matrix methods.

\begin{tabular}{|c|c|c|c|c|c|}
\hline & & $\overline{\mathrm{UT}}$ & $\overline{\mathrm{CC}}$ & \multicolumn{2}{|c|}{ UCC } \\
\hline $\begin{array}{l}\text { Stiffness } \\
\text { Method }\end{array}$ & $\begin{array}{c}\text { Strain } \\
(\%)\end{array}$ & $\begin{array}{c}\text { Cauchy Stress } \\
\text { (\% error) }\end{array}$ & $\begin{array}{c}\text { Cauchy Stress } \\
\text { (\% error) }\end{array}$ & $\begin{array}{c}\text { Cauchy Stress } \\
\text { (\% error) }\end{array}$ & $\begin{array}{c}\mathrm{v} 31 \\
\text { (\% error) }\end{array}$ \\
\hline \multirow{5}{*}{$\mathrm{J}-\mathrm{K}$} & 0 & 0.00 & 0.00 & 0.00 & \\
\hline & 10 & 7.07 & & & \\
\hline & 15 & & 1.16 & 274.37 & 63.52 \\
\hline & 30 & & 1.65 & 67.55 & 80.53 \\
\hline & 45 & & 15.23 & 14.79 & 82.62 \\
\hline \multirow{5}{*}{ PFD } & $\overline{0}$ & 0.00 & $\overline{0.00}$ & 0.00 & \\
\hline & 10 & 7.07 & & & \\
\hline & 15 & & 1.16 & 274.37 & 63.52 \\
\hline & 30 & & 1.65 & 67.55 & 80.53 \\
\hline & 45 & & 15.23 & 14.79 & 82.62 \\
\hline \multirow{5}{*}{ DPF } & 0 & 0.00 & 0.00 & 0.00 & \\
\hline & 10 & 7.07 & & & \\
\hline & 15 & & 1.16 & 274.37 & 63.52 \\
\hline & 30 & & 1.65 & 67.55 & 80.53 \\
\hline & 45 & & 15.23 & 14.79 & 82.62 \\
\hline
\end{tabular}

Table 6.1 shows that there is small error between the ABAQUS simulations and the experimental results for both UT and CC. The UCC data shows large variations between the experimental and simulated results for both stress and Poisson's ratios. As stated in Chapter 5, the three methods will result in the same stress response, which explains why all the values in Table 6.1 are the same between the methods. 
The stress-strain curves for compressive experimental protocols are presented in Figures 6.1 and 6.2. UT is omitted from these graphs since the general shape of the curve cannot be shown with only two data points.

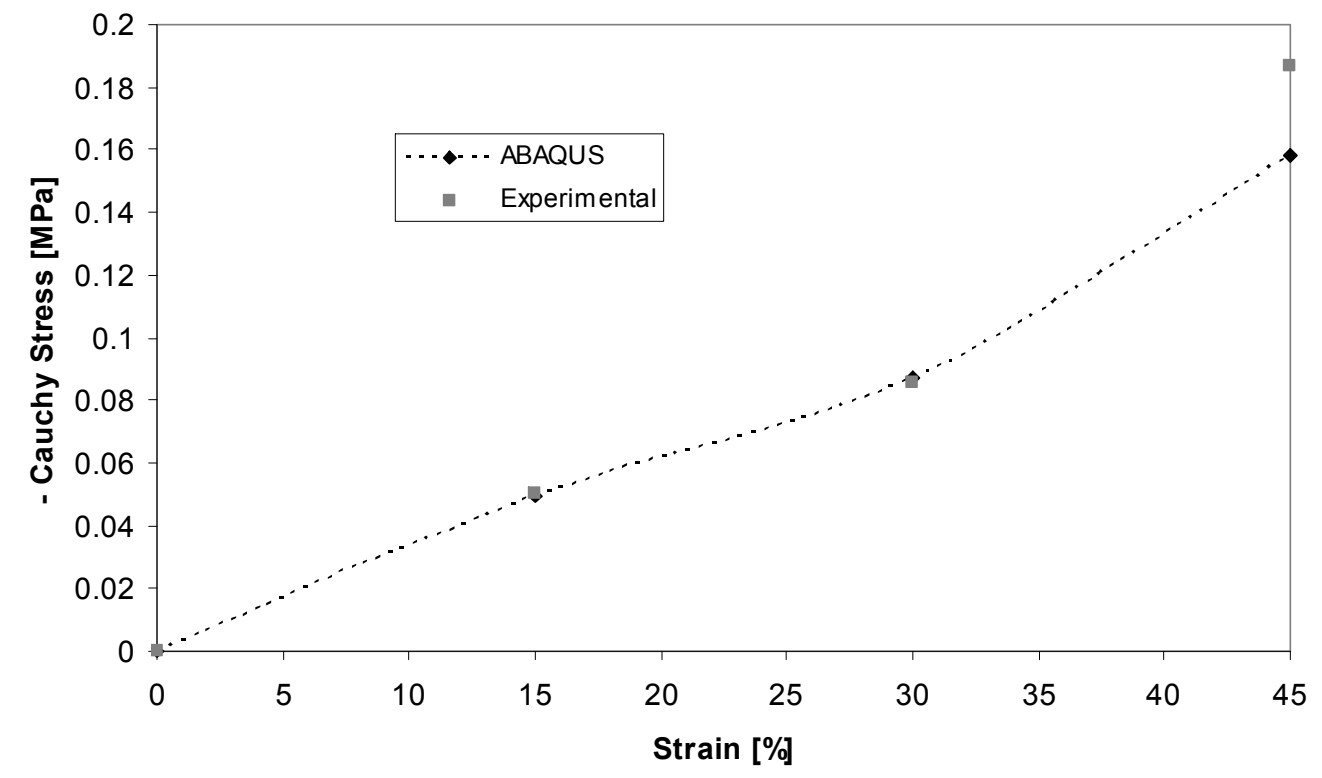

Figure 6.1: CC experimental and simulated data after optimization. The Cauchy stress is given for the direction of loading. CC experimental data was taken from P00 samples. 


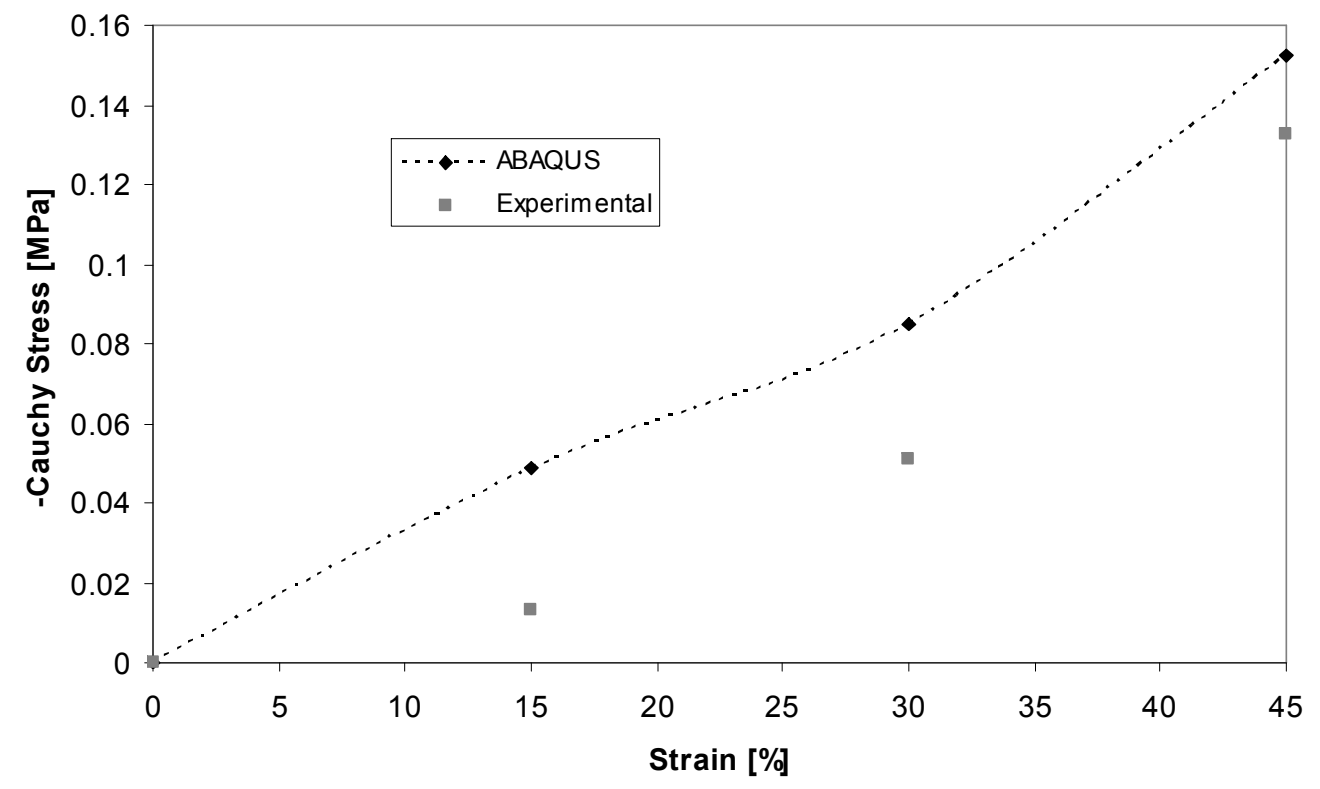

Figure 6.2: UCC experimental and simulated data after optimization. The Cauchy stress is given for the direction of loading. UCC experimental data was taken from D00 samples.

The CC results show that ABAQUS accurately predicts the CC stresses up to $30 \%$ strain and then under-predicts at $45 \%$ strain. ABAQUS over-predicts stresses for UCC at all points. It should be noted that there is little difference between the stress levels for UCC and CC in the ABAQUS simulations, while experimentally, the UCC Young's modulus is shown to be lower than the CC aggregate modulus. 


\section{Chapter 7}

\section{Discussion and Conclusions}

The first goal of this work was to implement an exact stiffness method into the ABAQUS UMAT subroutine. This was accomplished utilizing the J-K method. Additionally, this method utilized a stiffness matrix derived in terms of the entire solid matrix deformation $\left(\mathbf{C}^{S M}\right)$ as opposed to just constituent deformation $\left(\mathbf{C}^{\text {const }}\right)$. It was predicted that the J-K material stiffness matrix method would increase the robustness of the model, allowing it to converge to higher strains than with an approximation method. The simulations performed showed that switching to the J-K method increased the maximum convergence strains to levels above both the PFD and DPF methods.

Little change in maximum strain was noted in the $\mathrm{CC}$ simulations, due to the strict boundary conditions being implemented in this protocol. Additionally, using 1 element in these maximum convergence simulations decreased the de- 
grees of freedom on the system, thus decreasing the impact of possible errors due to material Jacobian approximations. It was found that the DPF and J-K method converged to the same strains in UT. The DPF method, as explained by Ficklin [3], utilizes the same $\widetilde{C}_{i j k l}$ as shown in equation (4.0.3). This makes the DPF code equivalent to the $\mathrm{J}-\mathrm{K}$ method minus the Jaumann co-rotational correction terms. Since there are no rotations in a direct UT protocol, the DPF method will behave similarly to the J-K method. The additional terms found in the J-K method, did however, assist in convergence in UCC. If rotational forces were implemented into the protocol, such as with a torsion test, the J-K method should outperform the DPF code.

Future work for this aim would be to test the results of the different methods under torsion protocols. The J-K method should also be applied to the CGFEM protocol developed in [3]. In the permeation protocol growth protocol, the cartilage samples curve after growth due to triggered mass deposition. This curvature created problems with convergence, possibly due to elemental rotations associated with curvature, that could be remedied by implementing the J-K method.

The second goal of this work was to implement material parameters that accurately predicted mechanical properties for day zero bovine calf $\mathrm{AC}$, based of gathered experimental data. Sufficient experimental data was gathered as a part 
of this work and the material parameter determination performed according to this data. This minimization was accomplished for both UT and CC protocols, as shown in Table 7.1. The CC results were accurate from $0-30 \%$ strains, but lower than experimental at $45 \%$. These simulations utilized a linear stress-strain relationship in terms of Lagrangian strain components [21] as opposed to a quadratic relationship [3], which were developed in [20]. Utilizing the nonlinear relation may more accurately predict the higher strain results, due to a faster increase in stress. The simulations also accurately showed a stress softening behavior in CC that has been noted through experimental results [4].

Throughout the material parameter optimization study, the UCC tests were unable to be accurately simulated using values that represented UT and CC properties. It was noted that the UCC stresses were close to the CC stresses, when experimental results show that the UCC modulus is less than the $\mathrm{CC}$ aggregate modulus. The simulated Poisson's ratios were magnitudes lower than the experimental values. The energy that should have been contributing to lateral expansion in UCC simulations was being used to resist compression, thus resulting in a higher Young's modulus. As discussed in Sections 5.2 and 5.3, the Poisson's ratios increased greatly with decreasing $\gamma_{1}$ values, while showing small changes with modifications to $\alpha_{2}$. This suggests that the collagen component of the matrix dominates the lateral expansion in UCC. 
Future work for this aim needs to focus on the optimization of day zero simulations for UCC. CC optimization took priority in this work due to its relationship with the permeation experimental protocol; however, to fully capture the material behavior of $\mathrm{AC}$, the UCC behavior needs to be accounted for.

Also, in this work the $\mu$ parameter was redefined to be in terms of the SM. Future work may work towards defining the GAG related molecular energies more quantitatively as opposed to the generalization proposed here. Triphasic models have previously been proposed to account for these ionic forces present in the solution [42]. Molecular models may accurately be able to approximate the contributions of the $\mu$ parameter on a more detailed level. They would be able to account for the molecular interactions between the collagen, proteoglycan, and remaining solid matrix components.

This work also optimized the $\mu$ parameter while leaving the $\gamma$ parameter constant. This was a valid approximation since the $\mu$ term had a small effect of the tensile results, while leaving $\gamma$ the same (Appendix D); however, future work should simultaneously modify $\mu$ and $\gamma$ to reach an optimized value for all parameters.

Overall, this work accomplished the goals of $\mathrm{J}-\mathrm{K}$ implementation and material parameter quantification. The J-K implementation broadens the applicability of the CGFEM to more loading and growth cases due to its superior conver- 
gence ability. The results of the parameter optimization provide an accurate baseline for growth and mechanical models of UT and CC protocols. As stated in the Chapter 2, the permeation protocol was successfully implemented experimentally and growth results obtained. For this reason, future research should model the experimental growth results using the modified CGFEM from this work. This would allow for comparison between experimental and theoretical growth formulas and provide insight into the mechanisms of $\mathrm{AC}$ growth. 


\section{List of References}

1. Bingham, M.S., The development of a cartilage finite element growth model: a thesis, in Mechanical Engineering. 2005, California Polytechnic State University: San Luis Obispo.

2. Davol, A., et al., A nonlinear finite element model of cartilage growth. Biomech Model Mechanobiol, 2008. 7(4): p. 295-307.

3. Ficklin, T., Application of a cartilage growth finite element model: Simulation of steady-state fluid permeation experiments: a thesis, in Mechanical Engineering. 2007, California Polytechnic State University: San Luis Obispo.

4. Stender, M., Differential Regulation of Articular Cartilage Biomechanical and Biochemical Properties by IGF-1 and TGF- ${ }^{\circledR} 1$ During In Vitro Growth, in ASME Summer Bioengineering Conference. 2009: Lake Tahoe, CA.

5. Stockwell, R., Biology of Cartilage Cells. 1979, Cambridge: Cambridge University Press.

6. Moody, H.R., et al., In vitro degradation of articular cartilage: does trypsin treatment produce consistent results? J Anat, 2006. 209(2): p. 259-67. 
7. Mauck, R.L., et al., Functional tissue engineering of articular cartilage through dynamic loading of chondrocyte-seeded agarose gels. J Biomech Eng, 2000. 122(3): p. 252-60.

8. Mow, V.C., et al., Biphasic creep and stress relaxation of articular cartilage in compression: Theory and experiments. J Biomech Eng, 1980. 102(1): p. 73-84.

9. Chahine, N.O., et al., Direct measurement of osmotic pressure of glycosaminoglycan solutions by membrane osmometry at room temperature. Biophys J, 2005. 89(3): p. 1543-50.

10. Temenoff, J.S. and A.G. Mikos, Review: tissue engineering for regeneration of articular cartilage. Biomaterials, 2000. 21(5): p. 431-40.

11. Williamson, A.K., et al., Tensile mechanical properties of bovine articular cartilage: variations with growth and relationships to collagen network components. J Orthop Res, 2003. 21(5): p. 872-80.

12. Hollander, A.P., et al., Increased damage to type II collagen in osteoarthritic articular cartilage detected by a new immunoassay. J Clin Invest, 1994. 93(4): p. 1722-32.

13. Williamson, A.K., A.C. Chen, and R.L. Sah, Compressive properties and function-composition relationships of developing bovine articular cartilage. J Orthop Res, 2001. 19(6): p. 1113-21.

14. Oungoulian, S.E., Articular cartilage constitutive modeling : a polyconvex strain energy function for proteoglycan and validation of a growth mixture model with collagen remodeling : a thesis, in Mechanical Engineering. 2007, California Polytechnic State University: San Luis Obispo.

15. Salter, R.B. and P. Field, The Effects of Continuous Compression on Living Articular Cartilage: An Experimental Investigation. Journal of Bone and Joint Surgery, 1960(42): p. 31-90. 
16. Cohen, B., W.M. Lai, and V.C. Mow, A transversely isotropic biphasic model for unconfined compression of growth plate and chondroepiphysis. J Biomech Eng, 1998. 120(4): p. 491-6.

17. Buckwalter, J.A. and H.J. Mankin, Articular cartilage: tissue design and chondrocyte-matrix interactions. Instr Course Lect, 1998. 47: p. 477-86.

18. Setton, L.A., W. Zhu, and V.C. Mow, The biphasic poroviscoelastic behavior of articular cartilage: role of the surface zone in governing the compressive behavior. J Biomech, 1993. 26(4-5): p. 581-92.

19. Klisch, S.M., et al., A cartilage growth mixture model with collagen remodeling: validation protocols. J Biomech Eng, 2008. 130(3): p. 031006.

20. Klisch, S.M., A bimodular polyconvex anisotropic strain energy function for articular cartilage. J Biomech Eng, 2007. 129(2): p. 250-8.

21. Thomas, G., Development of a poroviscoelastic model for articular cartilage: a thesis, in Mechanical Engineering. 2008, California Polytechnic State University: San Luis Obispo.

22. Asanbaeva, A., Cartilage growth and remodeling: modulation of growth phenotype and tensile integrity: a dissertation, in Bioengineering. 2006, University of California, San Diego.

23. Asanbaeva, A., et al., Articular cartilage tensile integrity: modulation by matrix depletion is maturation-dependent. Arch Biochem Biophys, 2008. 474(1): p. 175-82.

24. Thomas, G. and C. Barthel, Design of an apparatus for in vitro tissue growth under steady-state permeation. 2006, California Polytechnic State University: San Luis Obispo.

25. Pazzano, D., et al., Comparison of chondrogensis in static and perfused bioreactor culture. Biotechnol Prog, 2000. 16(5): p. 893-6. 
26. Buschmann, M.D., et al., Stimulation of aggrecan synthesis in cartilage explants by cyclic loading is localized to regions of high interstitial fluid flow. Arch Biochem Biophys, 1999. 366(1): p. 1-7.

27. Kwan, M.K., et al., The effect of storage on the biomechanical behavior of articular cartilage--a large strain study. J Biomech Eng, 1992. 114(1): p. $149-53$.

28. Chen, A.C., et al., Depth- and strain-dependent mechanical and electromechanical properties of full-thickness bovine articular cartilage in confined compression. J Biomech, 2001. 34(1): p. 1-12.

29. Ficklin, T., et al., Articular cartilage mechanical and biochemical property relations before and after in vitro growth. J Biomech, 2007. 40(16): p. 3607-14.

30. Spencer, A.J., Continuum Mechanics. 1980, New York, NY: Longman.

31. Buschmann, M.D. and A.J. Grodzinsky, A molecular model of proteoglycan-associated electrostatic forces in cartilage mechanics. J Biomech Eng, 1995. 117(2): p. 179-92.

32. Oungoulian, S., et al., Extended two compartmental swelling stress model and isotropic Cauchy stress equation for articular cartilage proteoglycans, in ASME Summer Bioengineering Conference. 2007.

33. Vena, P., Personal Communication. 2009.

34. Ateshian, G., H. Wang, and W. Lai, Role of interstitial fluid pressurization and surface porosities on the boundary friction of articular cartilage, in The 1997 Joint ASME/STLE/IMechE World Tribology Conference. 1997.

35. Mansour, J.M. and V.C. Mow, The permeability of articular cartilage under compressive strain and at high pressures. J Bone Joint Surg Am, 1976. 58(4): p. 509-16. 
36. ABAQUS/Standard user's manual: version 6.4. 2003.

37. Weiss, J.A., B.N. Maker, and S. Govindjee, Finite element implementation of incompressible, transversely isotropic hyperelasticity. Computer Methods in Applied Mechanics and Engineering, 1996. 135(1-2): p. 107-128.

38. Bardet, J.P., Finite-Element Analysis of Surface Instability in Hypoelastic Solids. Computer Methods in Applied Mechanics and Engineering, 1990. 78(3): p. 273-296.

39. Wilson, W., C.C. van Donkelaar, and J.M. Huyghe, A comparison between mechano-electrochemical and biphasic swelling theories for soft hydrated tissues. J Biomech Eng, 2005. 127(1): p. 158-65.

40. Mow, V.C. and A. Ratcliffe, Structure and function of articular cartilage and meniscus, in Basic Orthopaedic Biomechanics, W.C. Hayes, Editor. 1997, Raven Press: New York, NY. p. 113-178.

41. Zhu, W., et al., Viscoelastic shear properties of articular cartilage and the effects of glycosidase treatments. J Orthop Res, 1993. 11(6): p. 771-81.

42. Lai, W.M., J.S. Hou, and V.C. Mow, A triphasic theory for the swelling and deformation behaviors of articular cartilage. J Biomech Eng, 1991. 113(3): p. 245-58. 


\section{Appendix A}

\section{Derivations}

A.1 Partial Derivative of a Tensor with Respect to its

Inverse

Determine: $\frac{\partial C_{A B}^{-1}}{\partial C_{C D}}$ :

$$
\begin{aligned}
C_{A M}^{-1} C_{M N} & =\delta_{A N} \\
\frac{\partial}{\partial C_{C D}}\left(C_{A M}^{-1} C_{M N}\right) & =0 \\
\frac{\partial C_{A M}^{-1}}{\partial C_{C D}} C_{M N}+C_{A M}^{-1} \frac{\partial C_{M N}}{\partial C_{C D}} & =0 \\
\frac{\partial C_{A M}^{-1}}{\partial C_{C D}} C_{M N} & =-\frac{1}{2} C_{A M}^{-1}\left(\delta_{M C} \delta_{N D}+\delta_{M D} \delta_{N C}\right)
\end{aligned}
$$


Multiplying both sides by $C_{N B}^{-1}$ yields:

$$
\begin{aligned}
\frac{\partial C_{A M}^{-1}}{\partial C_{C D}} C_{M N} C_{N B}^{-1} & =-\frac{1}{2} C_{A M}^{-1} C_{N B}^{-1}\left(\delta_{M C} \delta_{N D}+\delta_{M D} \delta_{N C}\right) \\
\frac{\partial C_{A M}^{-1}}{\partial C_{C D}} \delta_{M B} & =-\frac{1}{2}\left(C_{A C} C_{B D}+C_{A D} C_{B C}\right) \\
\frac{\partial C_{A B}^{-1}}{\partial C_{C D}} & =-\frac{1}{2}\left(C_{A C} C_{B D}+C_{A D} C_{B C}\right)
\end{aligned},
$$




\section{A.2 Linearization Analysis to Determine UCC Pois-}

\section{son's Ratio}

This linearization analysis was used to approximate the Poisson's ratio

that should be expected from the model. The linearization of Cauchy stress $(\mathbf{T})$ assumes small strains, $e$. Both the collagen and proteoglycan constituents needed to be linearized to approximate the behavior of the entire SM in UCC. It assumed for this analysis that $\mathbf{F}_{O}^{C O L}=\mathbf{F}_{O}^{P G}=\mathbf{I}$.

\section{A.2.1 Collagen Constituent}

The collagen equation for the linearization utilizes the Cauchy stress equivalent of the isotropic collagen contribution found in equation (3.2.2):

$$
\mathbf{T}^{O}=\frac{1}{J} \mu(\mathbf{B}-\mathbf{I})
$$

where,

$$
\mathbf{B}=\mathbf{F F}^{T}
$$

To linearize this equation, we utilize the displacement gradient tensor, $\mathbf{H}$, which can be used to define the strain, e:

$$
\mathbf{e}=\frac{1}{2}\left(\mathbf{H}+\mathbf{H}^{\mathbf{T}}\right)
$$


Also, $\mathbf{F}$ can be related to $\mathbf{H}$ through:

$$
\mathbf{F}=\mathbf{H}+\mathbf{I} .
$$

Using this relation $\mathbf{B}$ becomes:

$$
\mathbf{B}=(\mathbf{H}+\mathbf{I})\left(\mathbf{H}^{\mathrm{T}}+\mathbf{I}\right) .
$$

Neglecting the second order terms, B simplifies to:

$$
\mathbf{B} \approx \mathbf{H}+\mathbf{H}^{\mathrm{T}}+\mathbf{I} .
$$

Combining equations (A.2.6), (A.2.3), and (A.2.1) the Cauchy stress equation for the isotropic collagen term becomes:

$$
\mathbf{T}^{O} \approx \frac{1}{J} 2 \mathbf{e} \mu .
$$

$1 / J$ can then linearize to the $1-\operatorname{tr}(\mathbf{e})$, where $\operatorname{tr}(\mathbf{e})$ is the trace of $\mathbf{e}$. Combining this result with equation (A.2.7) and neglecting the second order terms, the isotropic collagen term linearizes to:

$$
\mathbf{T}^{O} \approx 2 \mathbf{e} \mu .
$$

\section{A.2.2 Proteoglycan Constituent}

The general form of the Cauchy stress (taken from equation (3.2.8)) for the proteoglycan constituent can be taken to be: 


$$
\mathbf{T}^{P G}=\left(\frac{-\alpha_{1}^{*}}{\left(J^{P G}\right)^{\alpha_{2}}}\right) \mathbf{I} .
$$

By linearizing $J$, equation (A.2.8) becomes:

$$
\mathbf{T}^{P G} \approx\left(-\alpha_{1}^{*}(1-\operatorname{tr}(\mathbf{e}))^{\alpha_{2}}\right) \mathbf{I} .
$$

Utilizing a Taylor Series expansion, while neglecting higher order terms, the $(1-\operatorname{tr}(\mathbf{e}))^{\alpha_{2}}$ term becomes:

$$
(1-\operatorname{tr}(\mathbf{e}))^{\alpha_{2}} \approx 1^{\alpha_{2}}+\frac{\alpha_{2}(1-\operatorname{tr}(\mathbf{e}))^{\alpha_{2}-1}(-1)}{1 !} \operatorname{tr}(\mathbf{e})+\ldots
$$

This simplifies to:

$$
(1-\operatorname{tr}(\mathbf{e}))^{\alpha_{2}} \approx 1-\alpha_{2} \operatorname{tr}(\mathbf{e})+\ldots
$$

The total contribution to Cauchy stress from the proteoglycan component then becomes:

$$
\mathbf{T}^{P G} \approx\left(-\alpha_{1}^{*}+\alpha_{1}^{*} \alpha_{2} \operatorname{tr}(\mathbf{e})\right) \mathbf{I},
$$

Utilizing the general form of the Cauchy stress for an isotropic, linear elastic material,

$$
\mathbf{T} \approx\left(\lambda \operatorname{tr}(\mathbf{e})+2 \mathbf{e} \mu=\alpha_{1}^{*} \alpha_{2} \operatorname{tr}(\mathbf{e})-\alpha_{1}^{*}\right) \mathbf{I},
$$

leads to 


$$
\lambda=\alpha_{1}^{*} \alpha_{2},
$$

where, $\lambda$ is the Lamé Parameter. The homogeneous linear isotropic material formula for Poisson's ratio can then be used as an approximation of the AC Poisson's ratio in UCC:

$$
v=\frac{\lambda}{2(\lambda+\mu)} .
$$

$\lambda$ and $\mu$ can then be placed in equation (A.2.15):

$$
v=\frac{\alpha_{1}^{*} \alpha_{2}}{2\left(\alpha_{1}^{*} \alpha_{2}+\mu\right)}=\frac{(0.05)(2.50)}{2((0.05)(2.50)+0.11)} .
$$

This yields an approximate Poisson's ratio of:

$$
v=0.34 \text {. }
$$




\section{Appendix B}

\section{Convergence Study}

An elemental convergence study was conducted once all elastic material parameters had been determined. Convergences were performed for both elastic and poroelastic models in both CC and UT for 1, 27, 64, and 343 elements. All the plots are shown in Figures B1 through B4. 


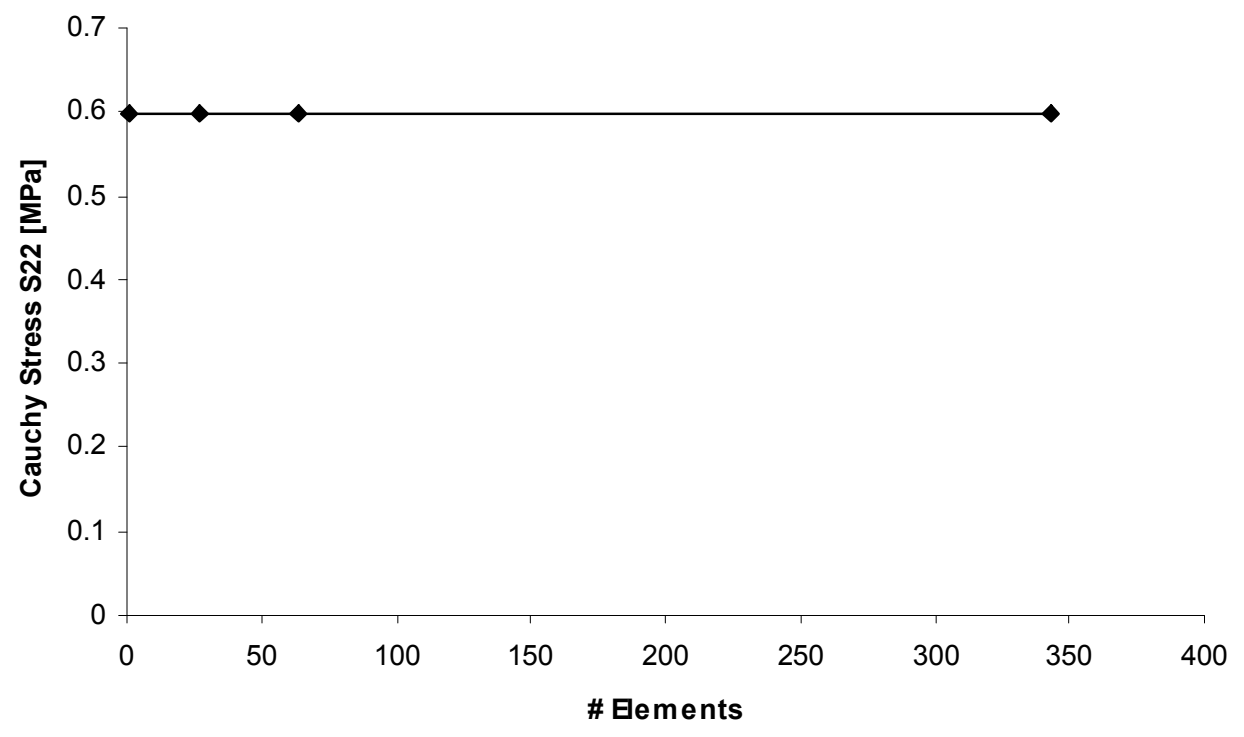

Figure B.1: Stress convergence for the elastic model in UT at 10\% strain.

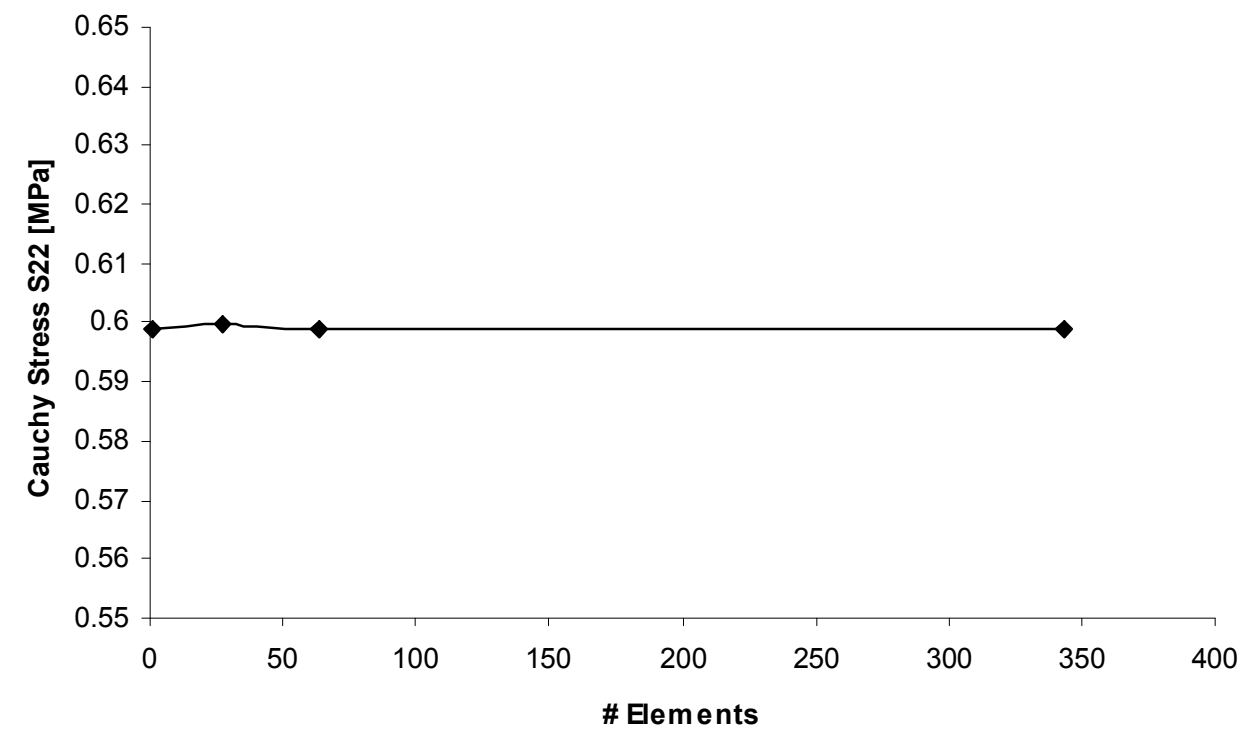

Figure B.2: Stress convergence for the poroelastic model in UT at 10\% strain. 


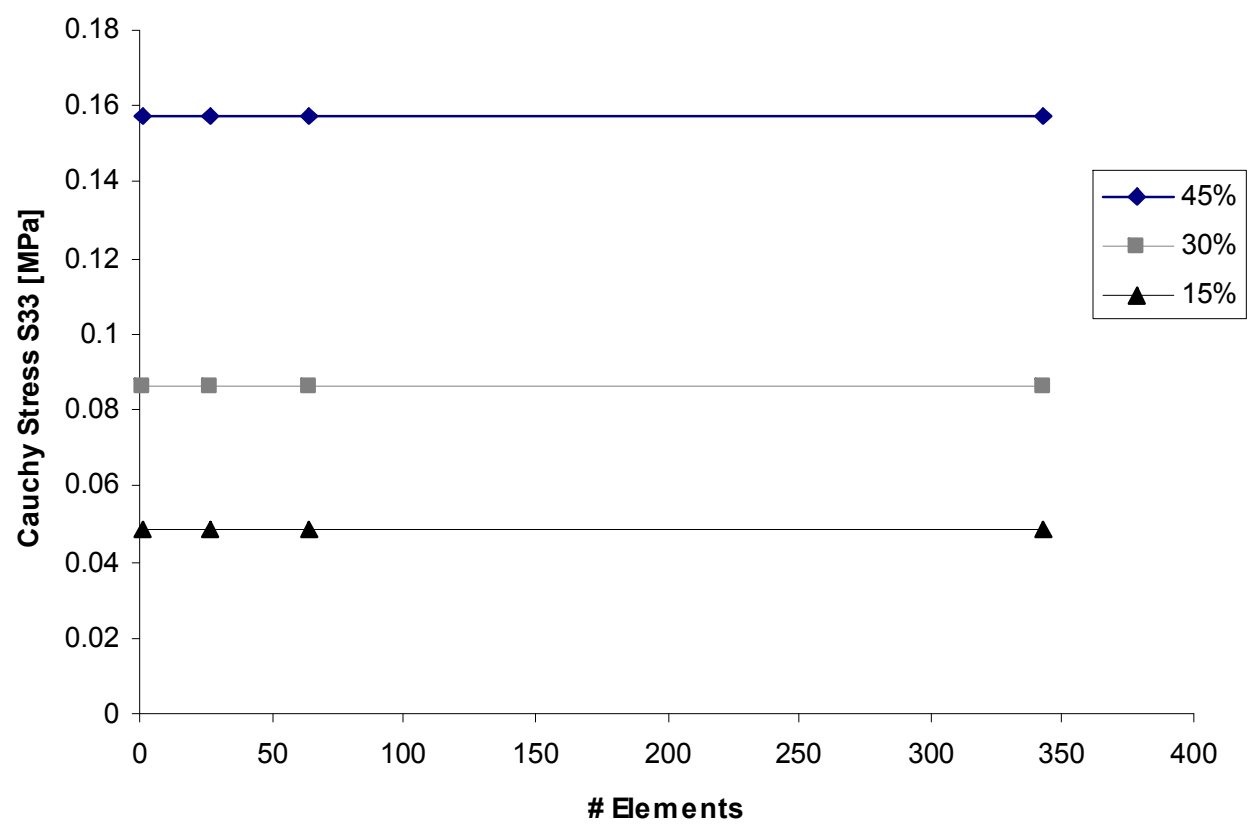

Figure B.3: Stress convergence for the elastic model in CC at $15 \%, 30 \%$, and $45 \%$ strains.

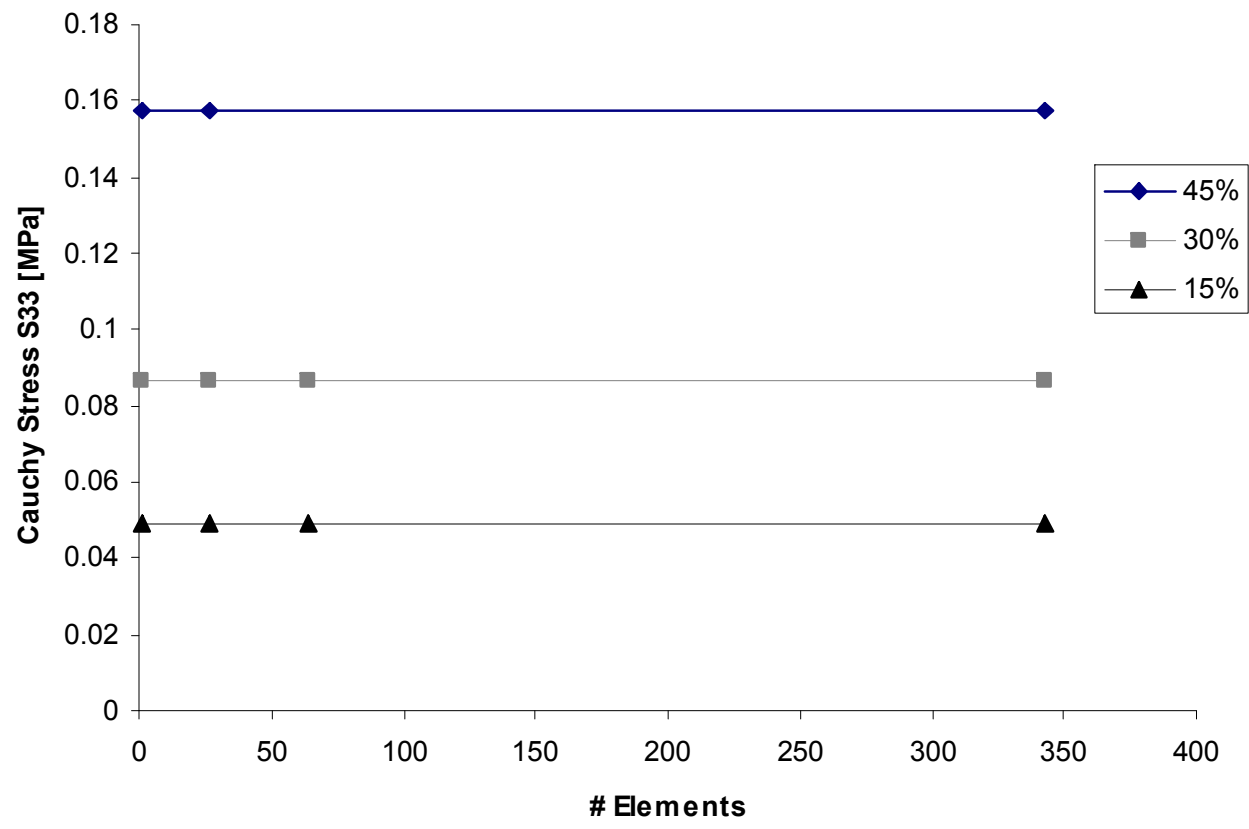

Figure B.4: Stress convergence for the poroelastic model in CC at $15 \%, 30 \%$, and $45 \%$ strains. 
As the convergence plots show, there is no advantage to increasing the simulations to more than 1 element. The amount of information being lost is negligible when compared to the computational time increase when using fewer elements. 


\section{Appendix $\mathrm{C}$}

\section{Initial Condition Iteration}

As stated, the $\mathbf{F}_{O}^{C O L}$ parameter needed to be iteratively solved for when material parameters were modified within the ABAQUS input file. The following procedure was utilized to determine the initial collagen deformation gradient:

1. Start with $\mathbf{F}_{O}^{C O L}=\mathbf{I}$.

2. Set all imposed displacements to zero.

3. Run the ABAQUS simulation using the chosen material parameters.

4. Open the ABAQUS .odb file in the front-end. Look at displacements in the 11 and 33 directions for the initial equilibrium step ("Wait_For_Eq" in current .inp files). 
5. The relationship, $\varepsilon=\frac{\delta}{l_{O}}$, where $\varepsilon$ is the Biot strain, is then applied to determine the next step. Plug-in the displacements recorded for $\delta$ and the length of the test specimen in the same direction as $\delta$. Note that the strain in direction $11=22$.

6. This yields an $\mathbf{F}^{B}$ that should be added to the $\mathbf{F}_{O}^{C O L}$ to yield the $\mathbf{F}_{O}^{C O L}$ for the next iteration.

7. Repeat until displacements reach $\sim 10^{-5}$ in all directions.

This was done due to the relatively small amount of simulations being performed. In the future it was be advantageous to create a custom program that worked with ABAQUS to solve for the collagen initial conditions. 


\section{Appendix D}

\section{Optimization Results}

\section{D.1 MD vs. EXT2 Model in Simulating CC}

MD and EXT2 GAG swelling stress models were tested to determine which represented the compression data more exactly. For all preliminary tests that were performed, both the MD and EXT2 models yielded stresses that were higher than those found experimentally. It was noted that for all the cases in these preliminary studies, that the MD model yielded lower stresses, and thus more closely approximated the experimental results. For this reason, the MD model was chosen for the remainder of the simulations. An example of these results in shown in Figure D.1. 


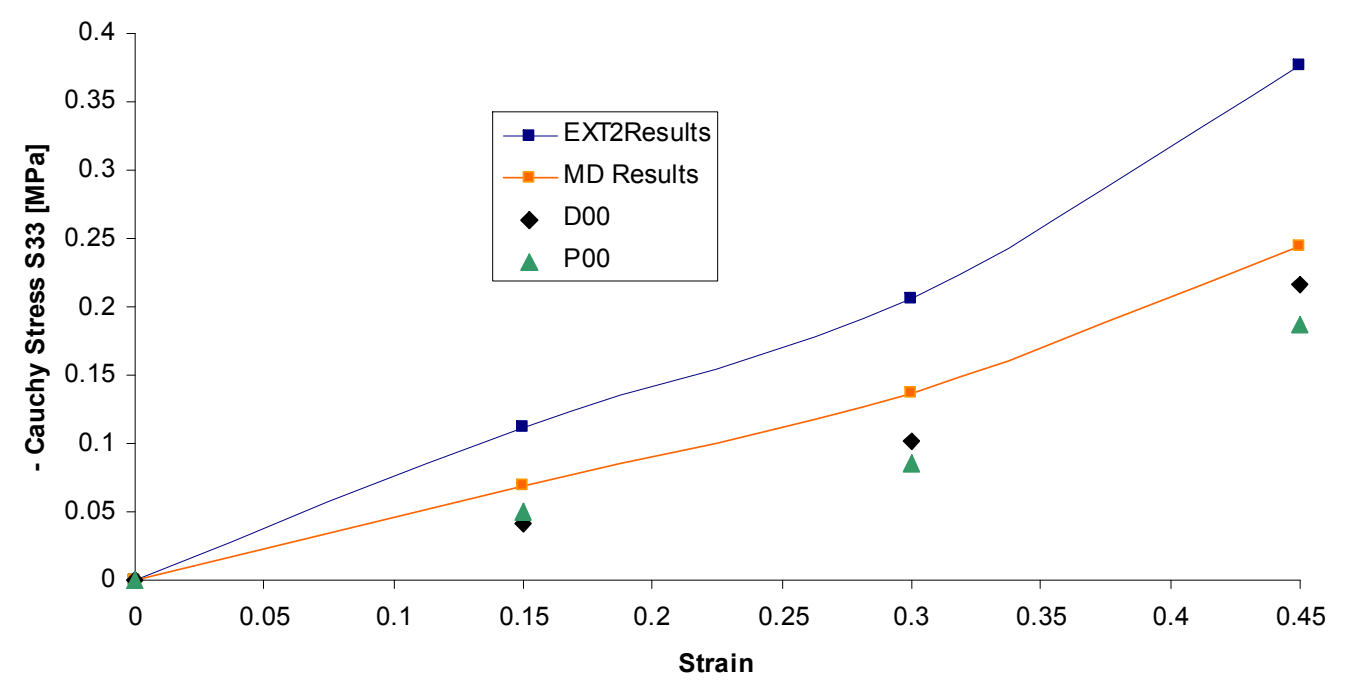

Figure D.1: MD and EXT2 model simulation comparisons to CC D00 and P00 experimental results.

\section{D.2 $\gamma_{1}$ Optimization Results}

$\gamma_{1}$ was varied between 0.25 and 1.50 to determine an optimal value for it within the mechanical simulations. This was also done to determine the impact of adjusting $\gamma_{1}$ on both compressive and tensile properties. 


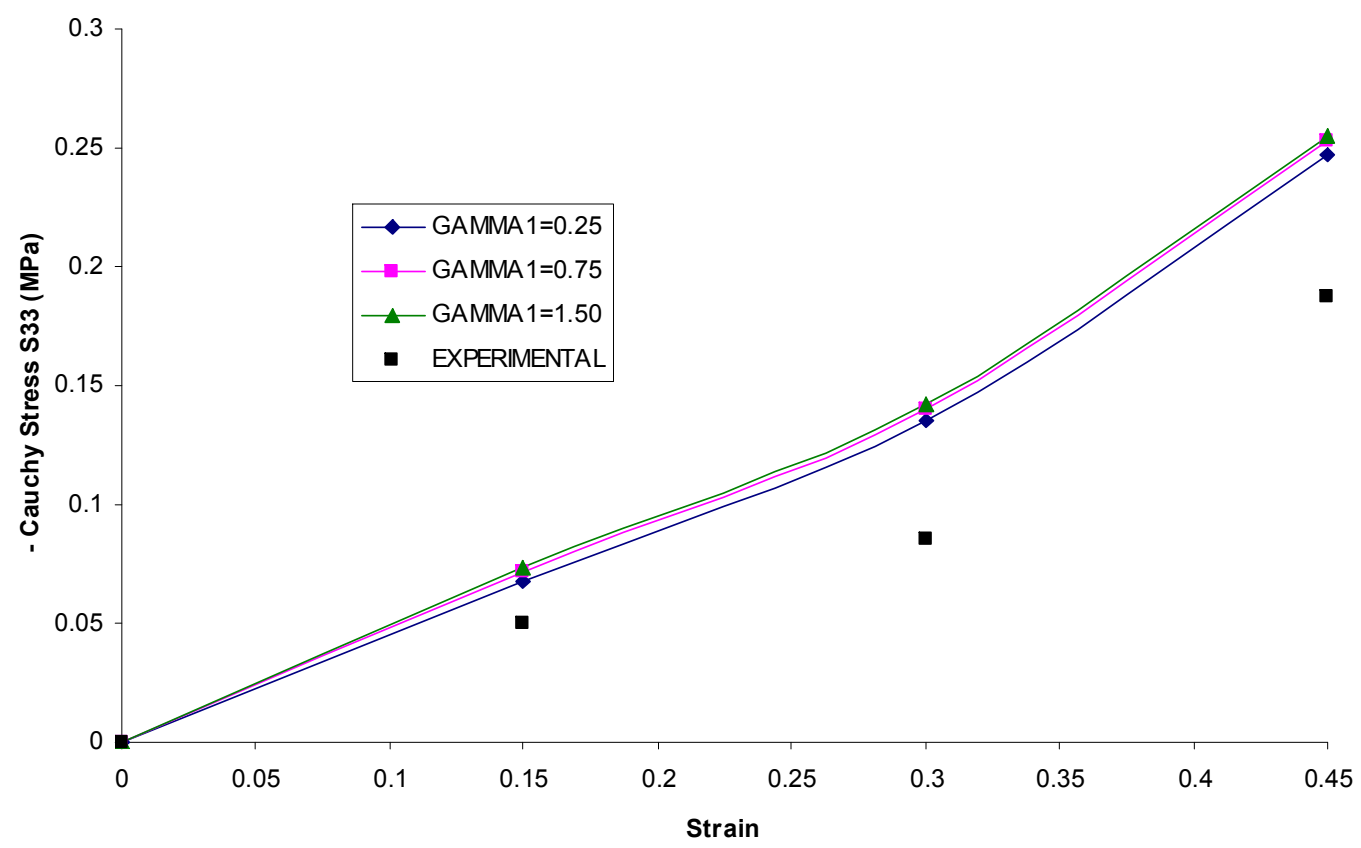

Figure D.2: Comparison of varying $\gamma_{1}$ values to experimental CC data using MD model.

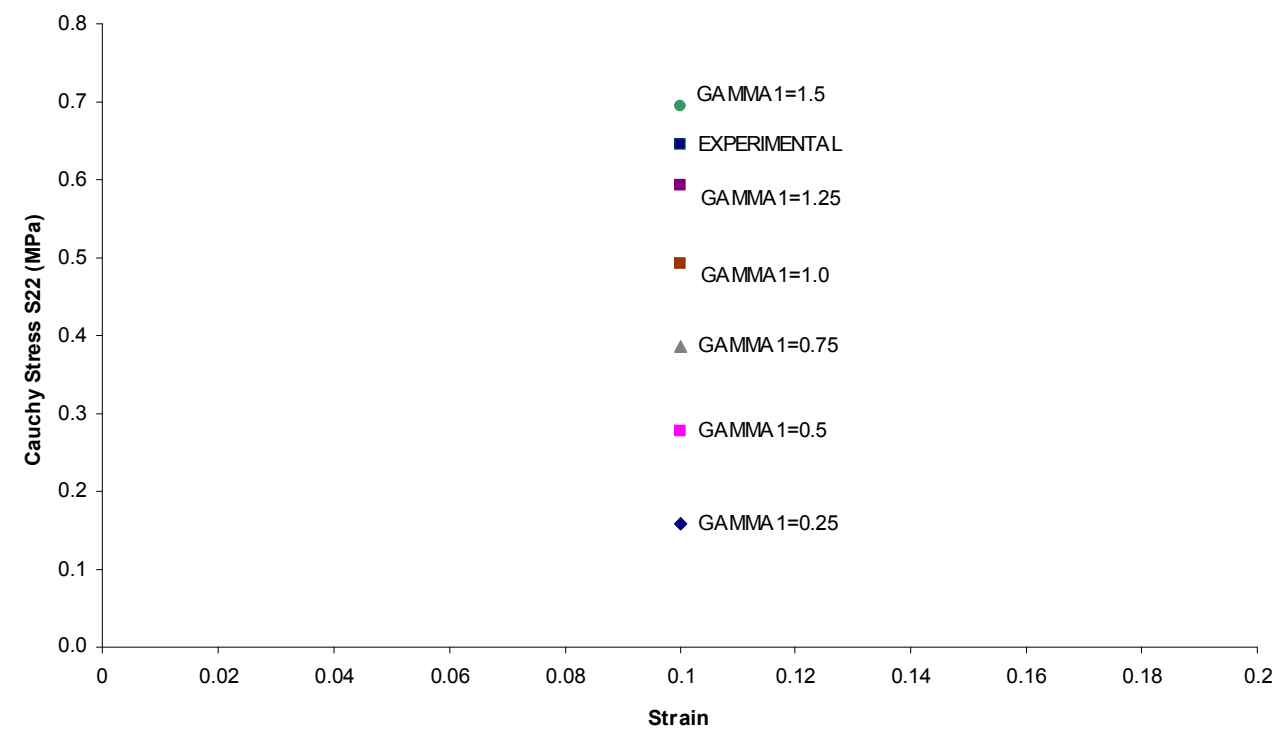

Figure D.3: Comparison of varying $\gamma_{1}$ values to experimental UT data using MD model. 
As can be seen from these plots, the $\gamma_{1}$ parameter had little effect on the CC modulus, but a large effect on the UT modulus. The final chosen value of $\gamma=1.38307$ is shown to be near the experimental data point found in Figure D.3.

\section{D.3 $\alpha_{2}$ Optimization Results}

A similar optimization to $\gamma_{1}$ was performed for $\alpha_{2}$, varying it between 2.3 and 2.8. Adjusting $\alpha_{2}$ yielded negligible changes to UT results. It did have a larger effect on the $\mathrm{CC}$ modulus, but was still not shown to be a major contributing parameter. For this reason, the $\alpha_{2}$ parameter was eventually taken from the work performed by Oungoulian [14], due to the reliability of his structural model.

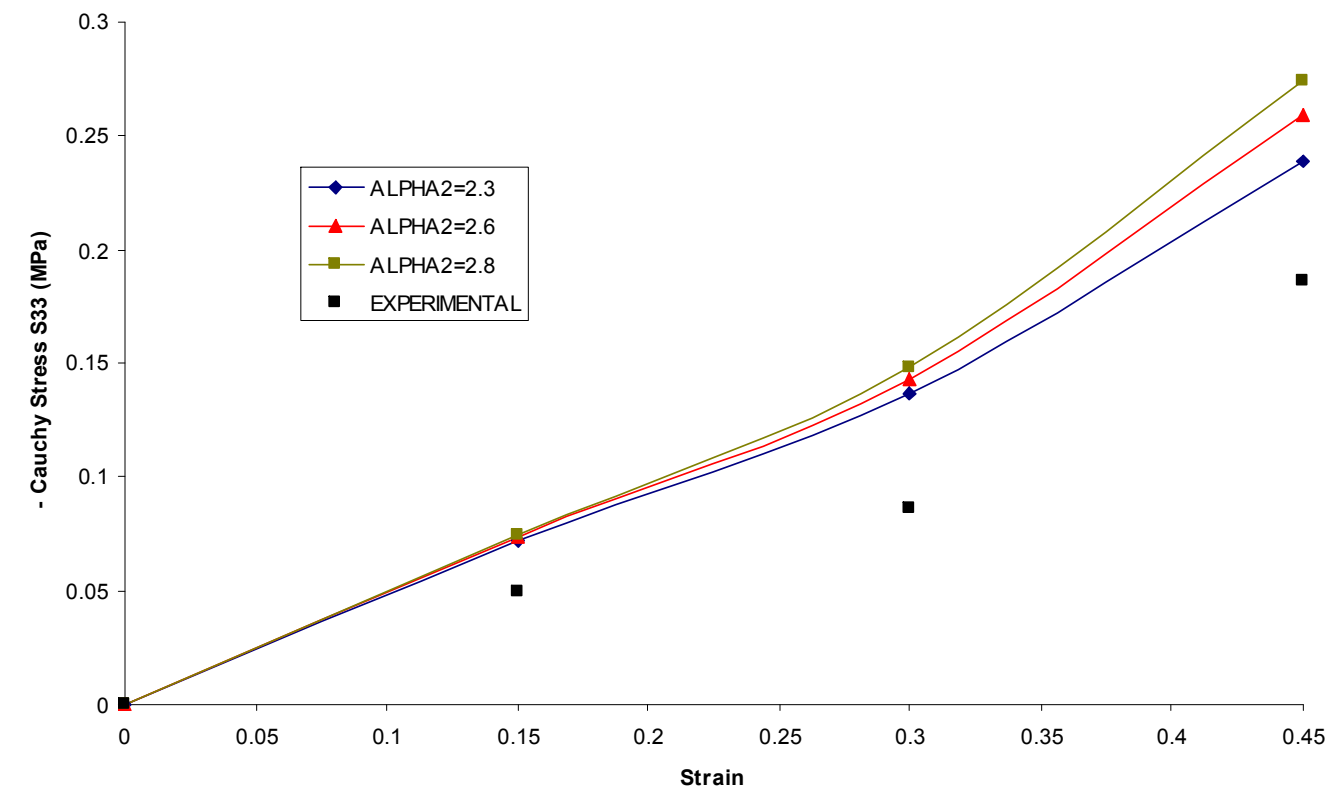

Figure D.4: Comparison of varying $\alpha_{2}$ values to experimental CC data using MD model. 


\section{D.4 $\mu$ Optimization Results with Error Analysis}

$\mu$ was optimized between values of 0.01 and 0.10 . This parameter had a significant impact on the $\mathrm{CC}$ mechanical response (Figure D.6). The $\mu$ parameter did not significantly alter the tensile stress response (Figure D.5), which allows for the assumption that modifying $\mu$ does not require simultaneous optimization of $\gamma_{1}$

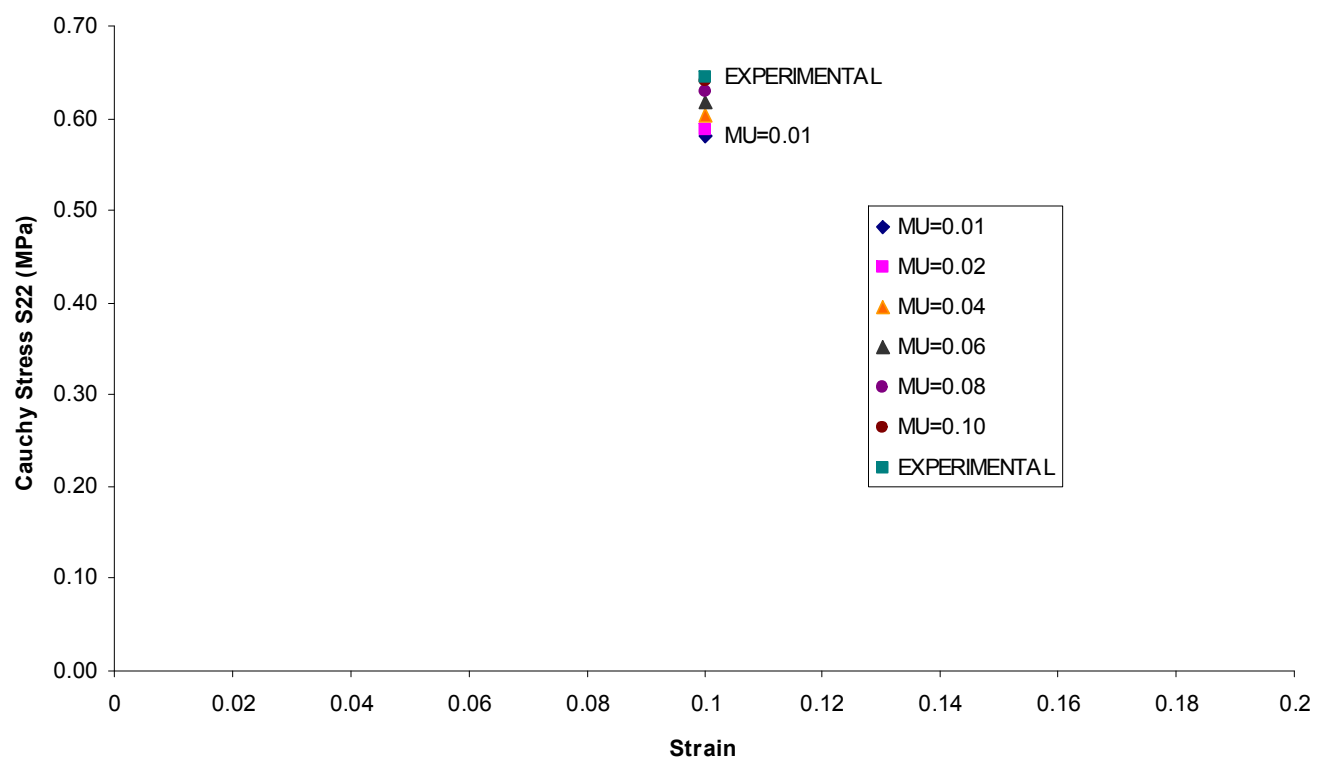

Figure D.5: Comparison of varying $\mu$ values to experimental UT data using MD model. 


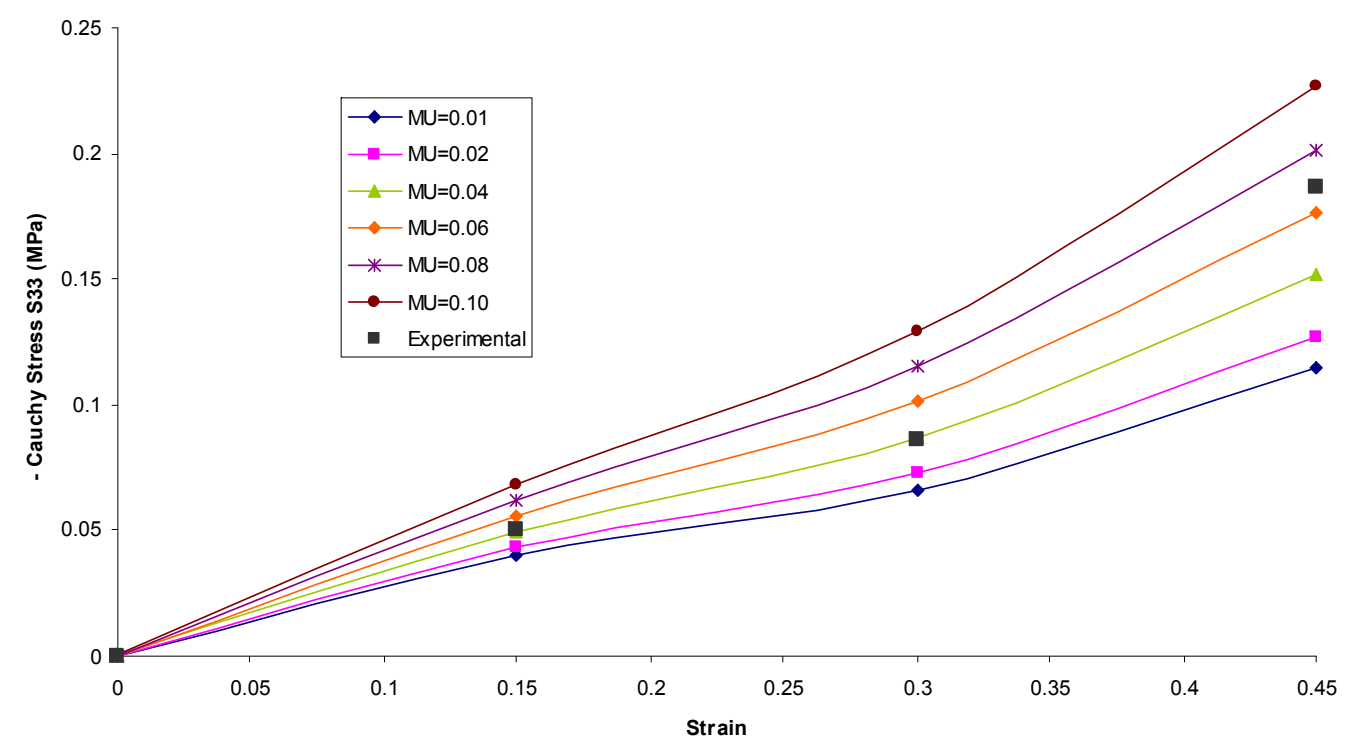

Figure D.6: Comparison of varying $\mu$ values to experimental $\mathrm{CC}$ data using MD model.

Figures D.5 and D.6 show that decreasing $\mu$ results in a decrease in the modulus of $\mathrm{AC}$ in $\mathrm{UT}$ and $\mathrm{CC}$. In order to match experimental results, the $\mathrm{CC}$ simulations needed to decrease in modulus, while the UT simulations needed to slightly increase in modulus. This created a trade-off when optimizing the system. Moving $\mu$ up or down would satisfy the experimental results of one of the protocols, but not the other. This created the need for an error analysis.

$30 \% \mathrm{CC}$ and $10 \% \mathrm{UT}$ strains were chosen as the strains to optimize around. The UT stresses at 10\% strain is roughly 1 order of magnitude larger than the CC stress at 30\% strain, which meant that the stresses needed to be normal- 
ized in order to equally account for both protocols. The overall raw data is shown in Table D.1.

Table D.1: Experimental data for UT, CC, and UCC tests. This data provides the experimental comparison for all simulations.

\begin{tabular}{|c|c|c|c|c|}
\cline { 2 - 5 } \multicolumn{1}{c|}{} & \multicolumn{3}{|c|}{ Cauchy Stress (Direction of Loading) } & $\begin{array}{c}\text { Poisson's } \\
\text { Ratio }\end{array}$ \\
\hline Strain & $\begin{array}{c}\text { UT } \\
\text { (D00) }\end{array}$ & $\begin{array}{c}\text { UCC } \\
(\mathrm{D} 00)\end{array}$ & $\begin{array}{c}\text { CC } \\
(\mathrm{P} 00)\end{array}$ & $\begin{array}{c}\text { UCC } \\
\text { v31 }\end{array}$ \\
\hline 0 & 0 & 0 & 0 & \\
\hline 0.1 & 0.6449 & & & \\
\hline 0.15 & & -0.01298 & -0.0500 & 0.0701 \\
\hline 0.3 & & -0.05074 & -0.0857 & 0.099 \\
\hline 0.45 & & -0.13270 & -0.1868 & 0.116 \\
\hline
\end{tabular}

The CC $30 \%$ value was divided by the UT $10 \%$ value to create a normalization value (0.1329). All CC 30\% simulation values were then divided by this constant to yield the normalized values shown in Table D.2. Sum of the square error (SSE) calculations were done relative to the experimental data presented in Table D.1, for all simulations. This is also presented in Table D.2.

Table D.2: Normalized CC $30 \%$ and UT $10 \%$ simulated results for variations of $\mu$ with SSE values shown.

\begin{tabular}{|c|c|c|c|c|c|c|}
\hline$\mu$ & $\begin{array}{c}\text { CC 30\% } \\
\text { Sim [MPa] }\end{array}$ & $\begin{array}{c}\text { CC 30\% } \\
1.1329\end{array}$ & $\begin{array}{c}\text { CC 30\% } \\
\text { SSE }\end{array}$ & $\begin{array}{c}\text { UT 10\% } \\
\text { Sim [MPa] }\end{array}$ & $\begin{array}{c}\text { UT 10\% } \\
\text { SSE }\end{array}$ & $\begin{array}{c}\text { SSE } \\
\text { Total }\end{array}$ \\
\hline 0.01 & 0.0709 & 0.5335 & 0.0124 & 0.5820 & 0.0040 & 0.0164 \\
\hline 0.02 & 0.0780 & 0.5868 & 0.0034 & 0.5898 & 0.0030 & 0.0064 \\
\hline 0.03 & 0.0851 & 0.6402 & 0.0000 & 0.5972 & 0.0023 & 0.0023 \\
\hline 0.04 & 0.0922 & 0.6936 & 0.0024 & 0.6043 & 0.0016 & 0.0040 \\
\hline 0.05 & 0.0993 & 0.7470 & 0.0104 & 0.6111 & 0.0011 & 0.0116 \\
\hline 0.06 & 0.1064 & 0.8004 & 0.0242 & 0.6176 & 0.0007 & 0.0249 \\
\hline 0.08 & 0.1206 & 0.9073 & 0.0689 & 0.6298 & 0.0002 & 0.0691 \\
\hline 0.10 & 0.1348 & 1.0143 & 0.1364 & 0.6412 & 0.0000 & 0.1365 \\
\hline
\end{tabular}


The SSE total was then used to determine the optimal $\mu$ value using a polynomial regression shown in Figure D.7.

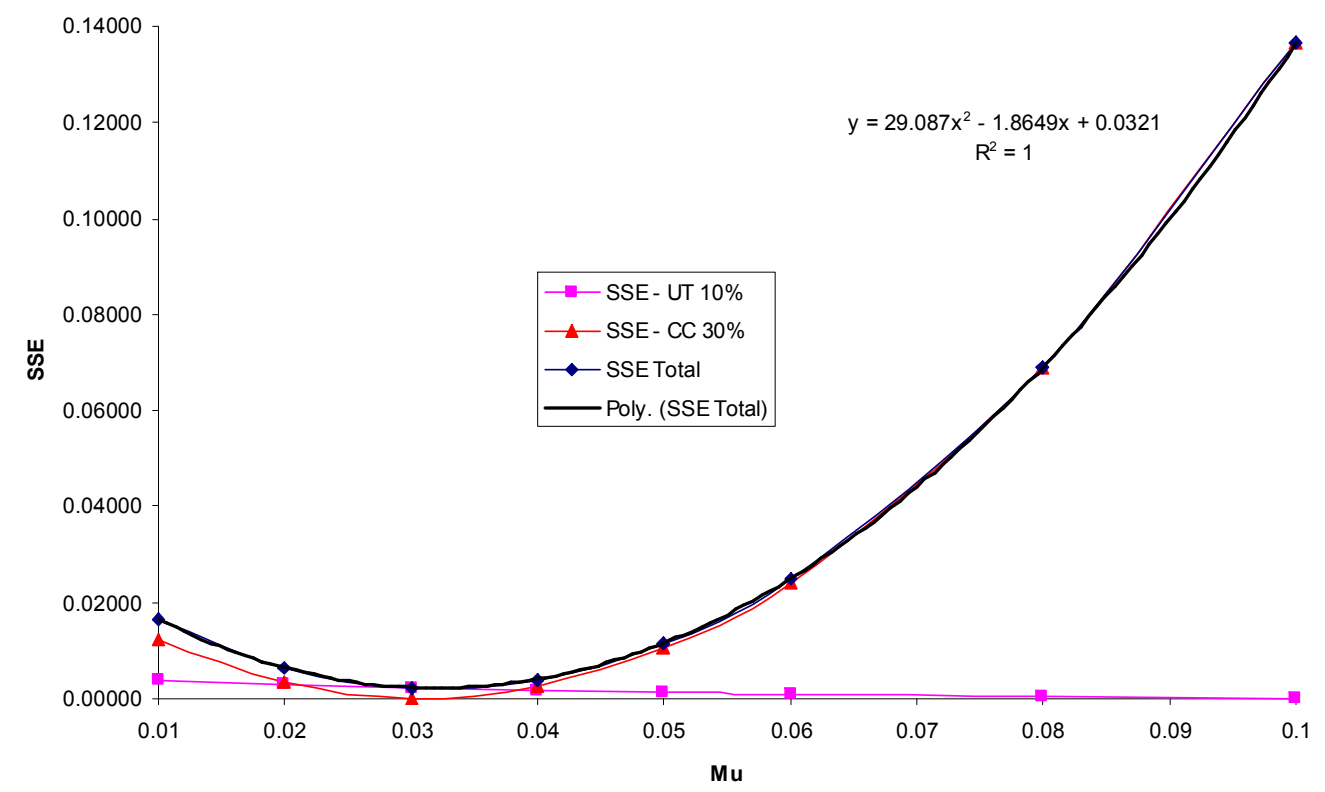

Figure D.7: Summary of SSE data for UT 10\% and CC 30\% simulations for varying $\mu$ with polynomial curve fit.

Figure D.7 shows the expected result of the UT error decreasing as $\mu$ approached 0.11 , as well as the inverse trend for CC error. Based on the curve-fit, the final $\mu$ value of 0.32 was chosen. The final simulated results are shown in Table D.3 using all optimized parameters. 
Table D.3: Simulated data for UT, CC, and UCC using optimized parameters.

\begin{tabular}{|c|c|c|c|c|}
\cline { 2 - 5 } \multicolumn{1}{c|}{} & \multicolumn{3}{c|}{ Cauchy Stress (Direction of Loading) } & $\begin{array}{c}\text { Poisson's } \\
\text { Ratio }\end{array}$ \\
\hline Strain & $\begin{array}{c}\text { UT } \\
\text { (D00) }\end{array}$ & $\begin{array}{c}\text { UCC } \\
\text { (D00) }\end{array}$ & $\begin{array}{c}\text { CC } \\
\text { (P00) }\end{array}$ & $\begin{array}{c}\text { UCC } \\
\text { v31 }\end{array}$ \\
\hline 0 & 0 & 0 & 0 & \\
\hline 0.1 & 0.5987 & & & \\
\hline 0.15 & & -0.04805 & -0.0489 & 0.025336 \\
\hline 0.3 & & -0.08442 & -0.0865 & 0.019153 \\
\hline 0.45 & & -0.15160 & -0.1576 & 0.020082 \\
\hline
\end{tabular}




\section{Appendix E}

\section{Codes and Inputs}

\section{E.1 ABAQUS UMAT Files}

\section{E.1.1 PFD}

This section contains the PFD.for code (pushed forward then differentiated), including the UMAT and VOIDRI subroutines, as used in this work.

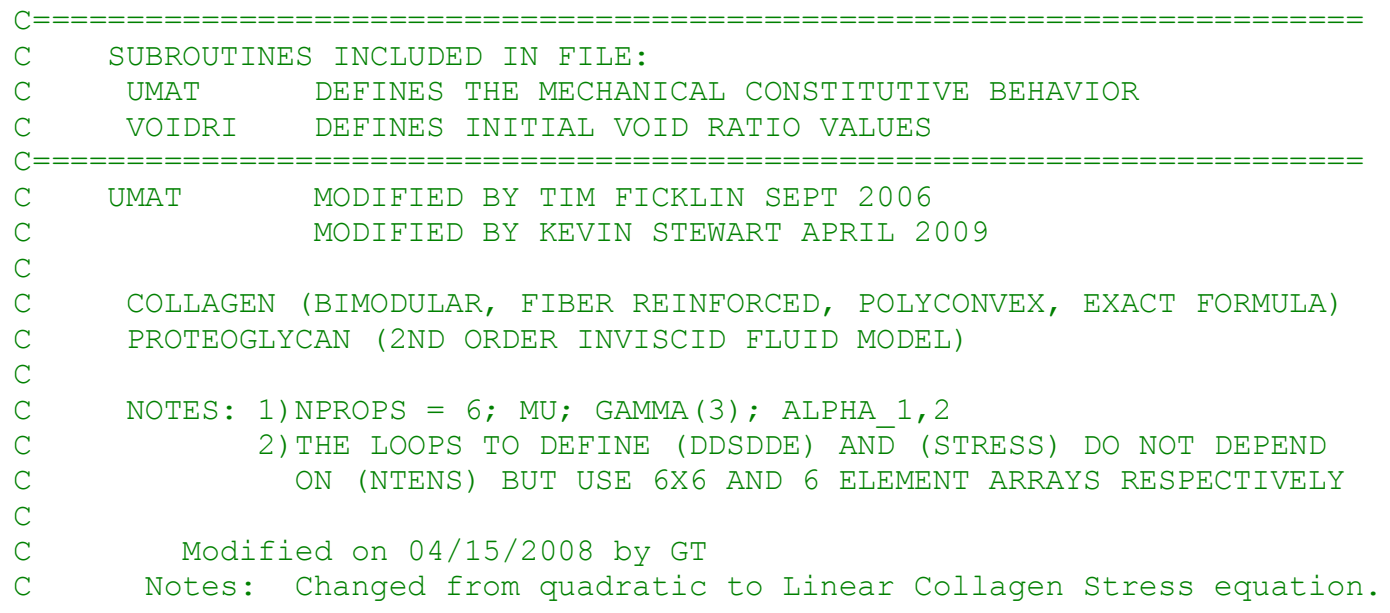




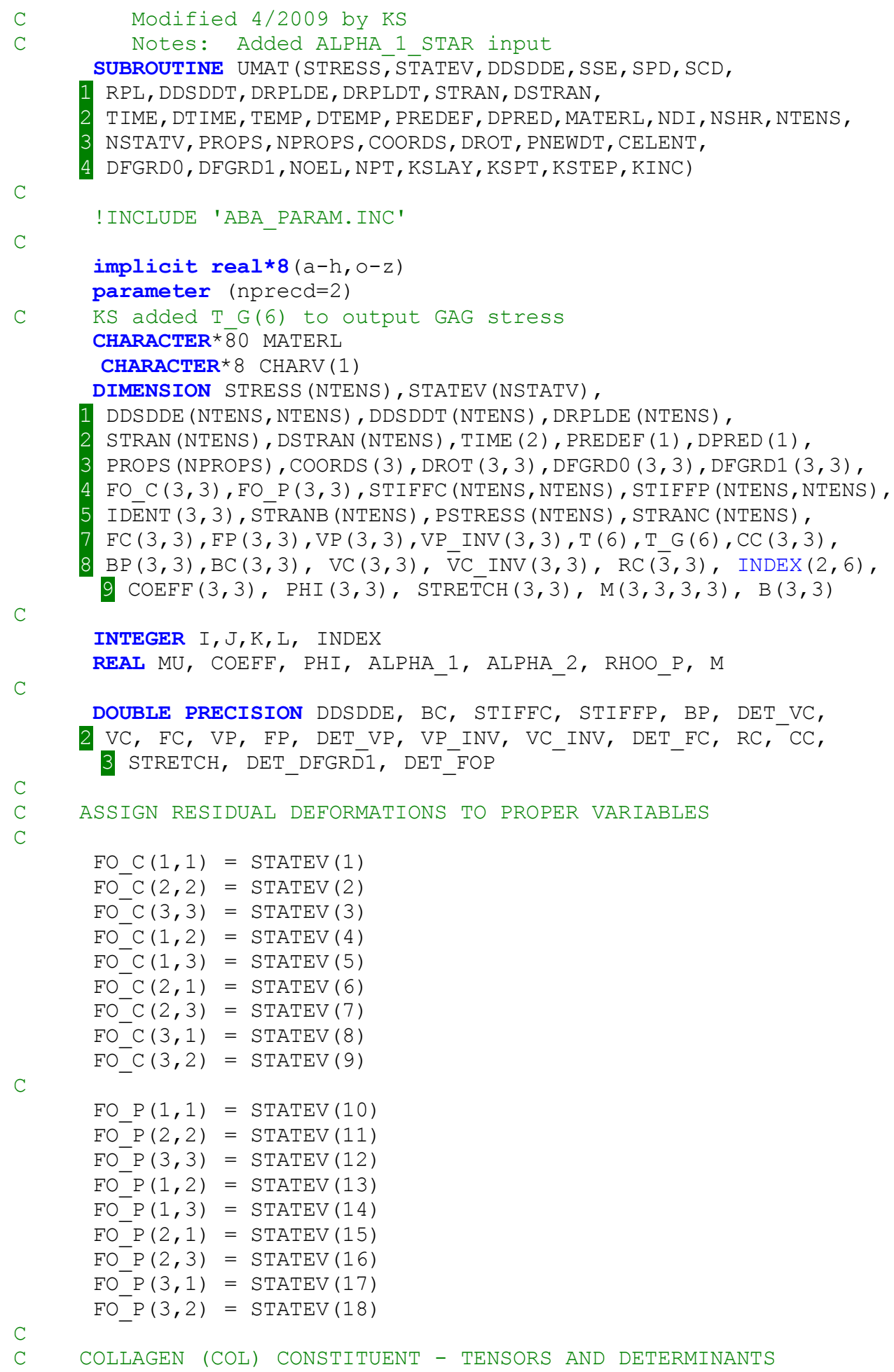




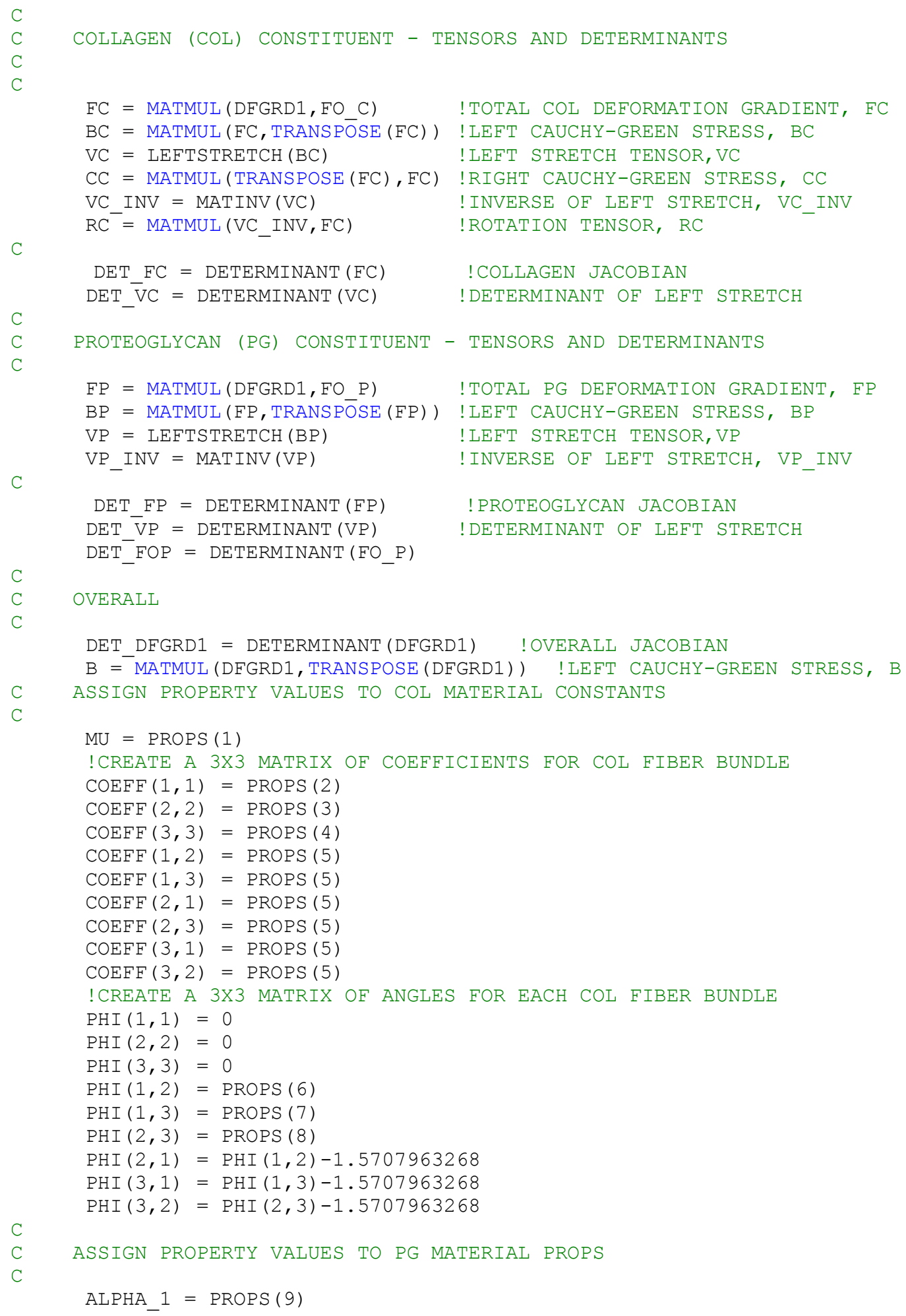




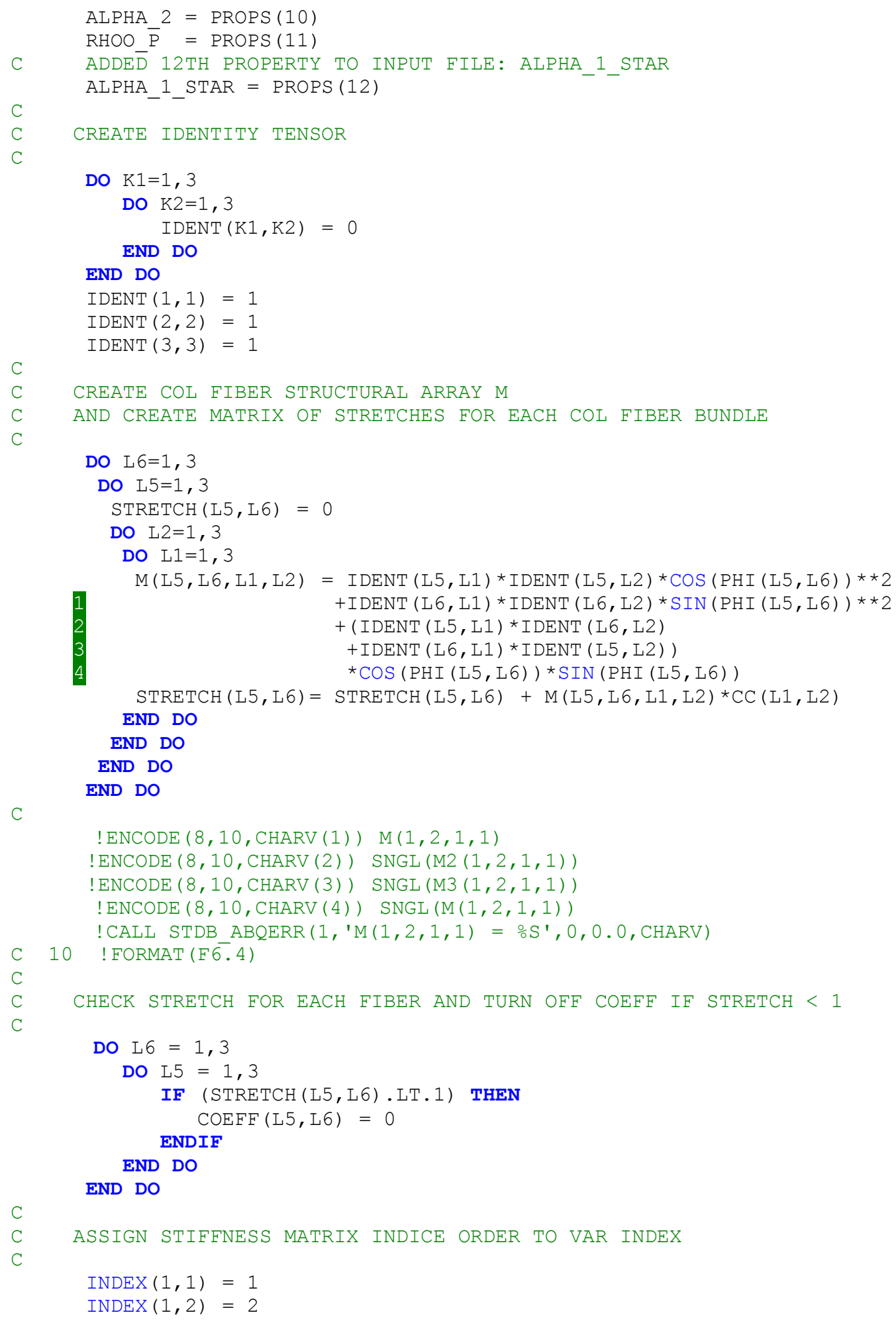




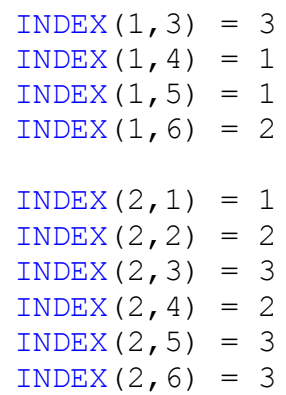




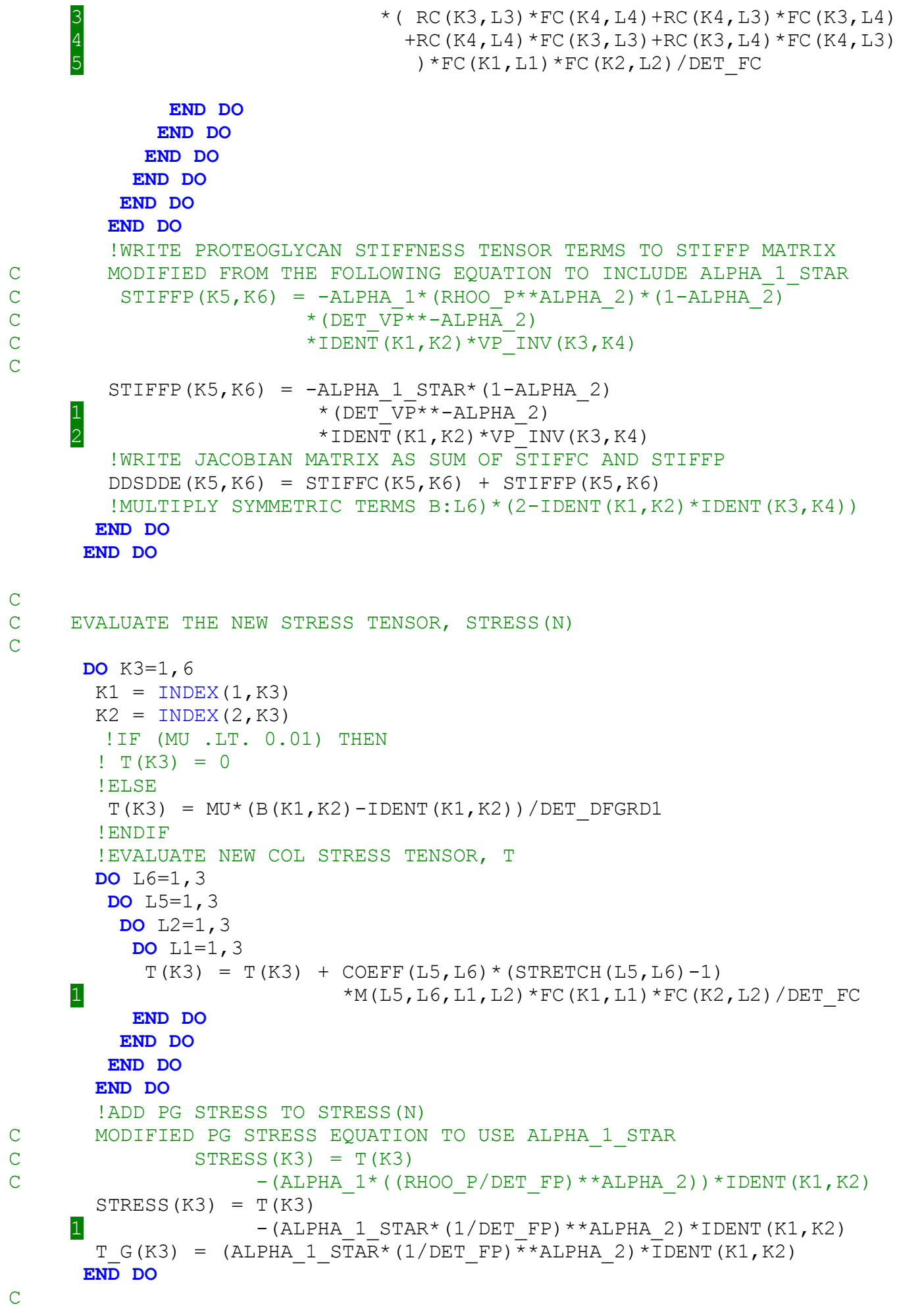




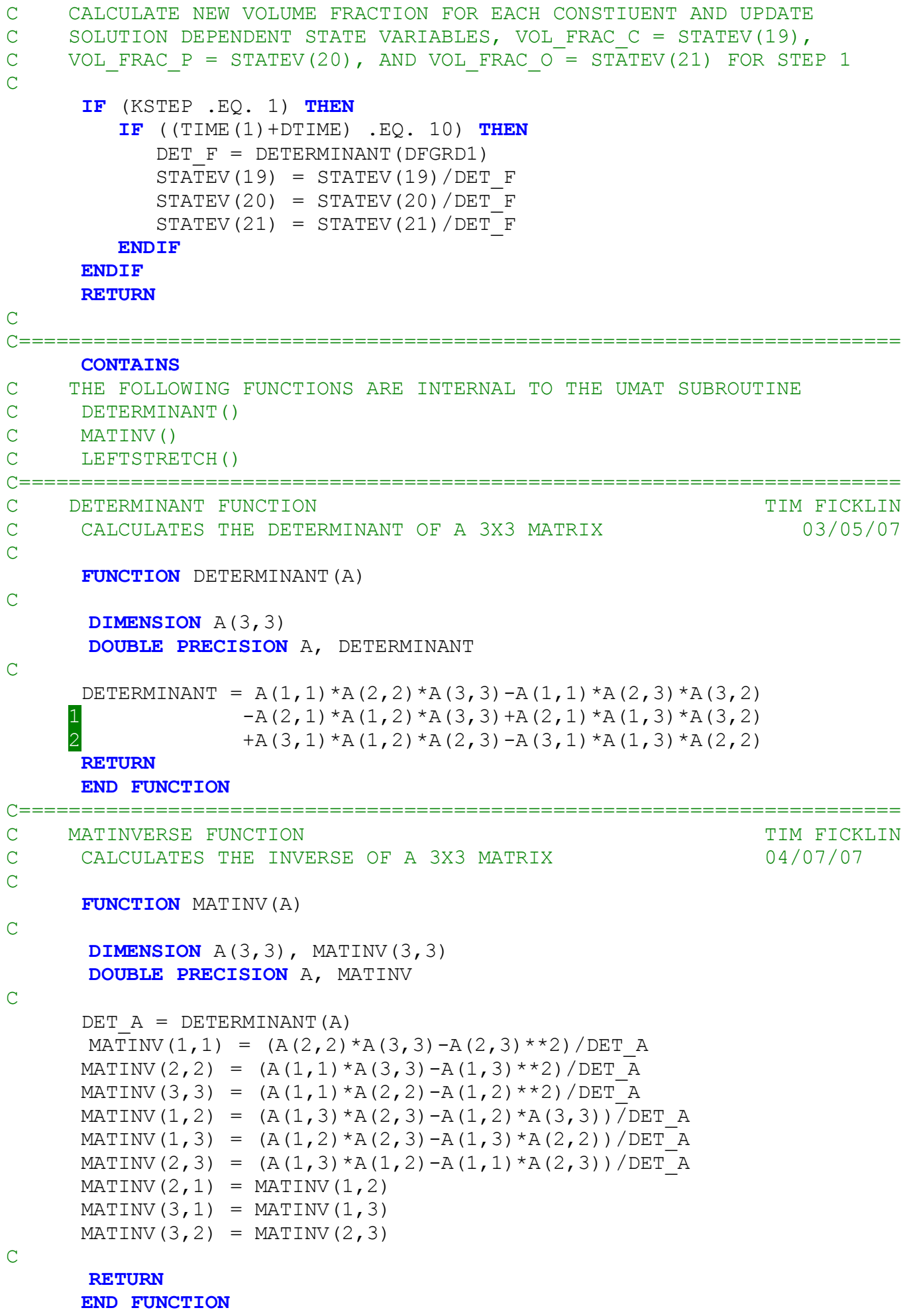




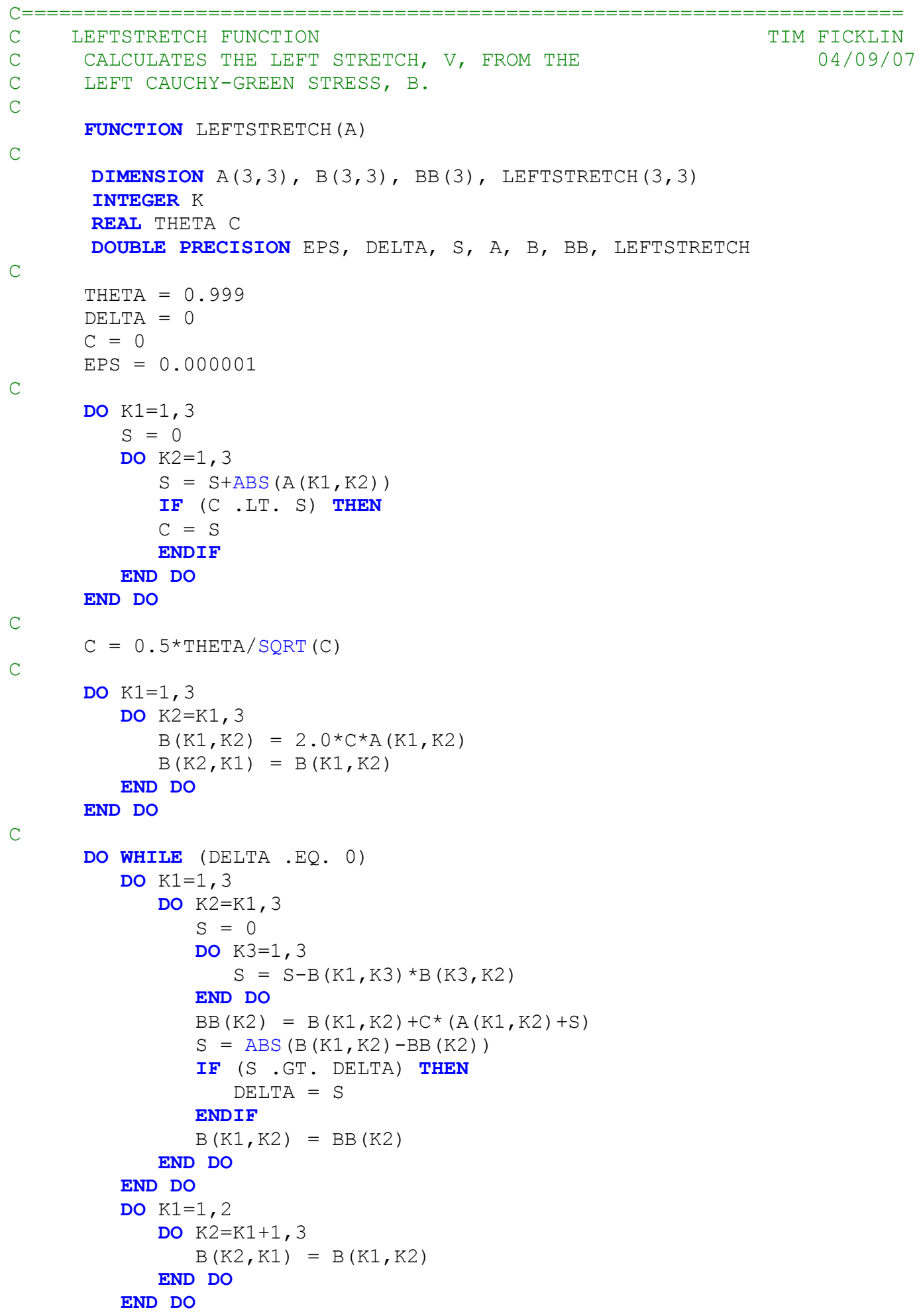




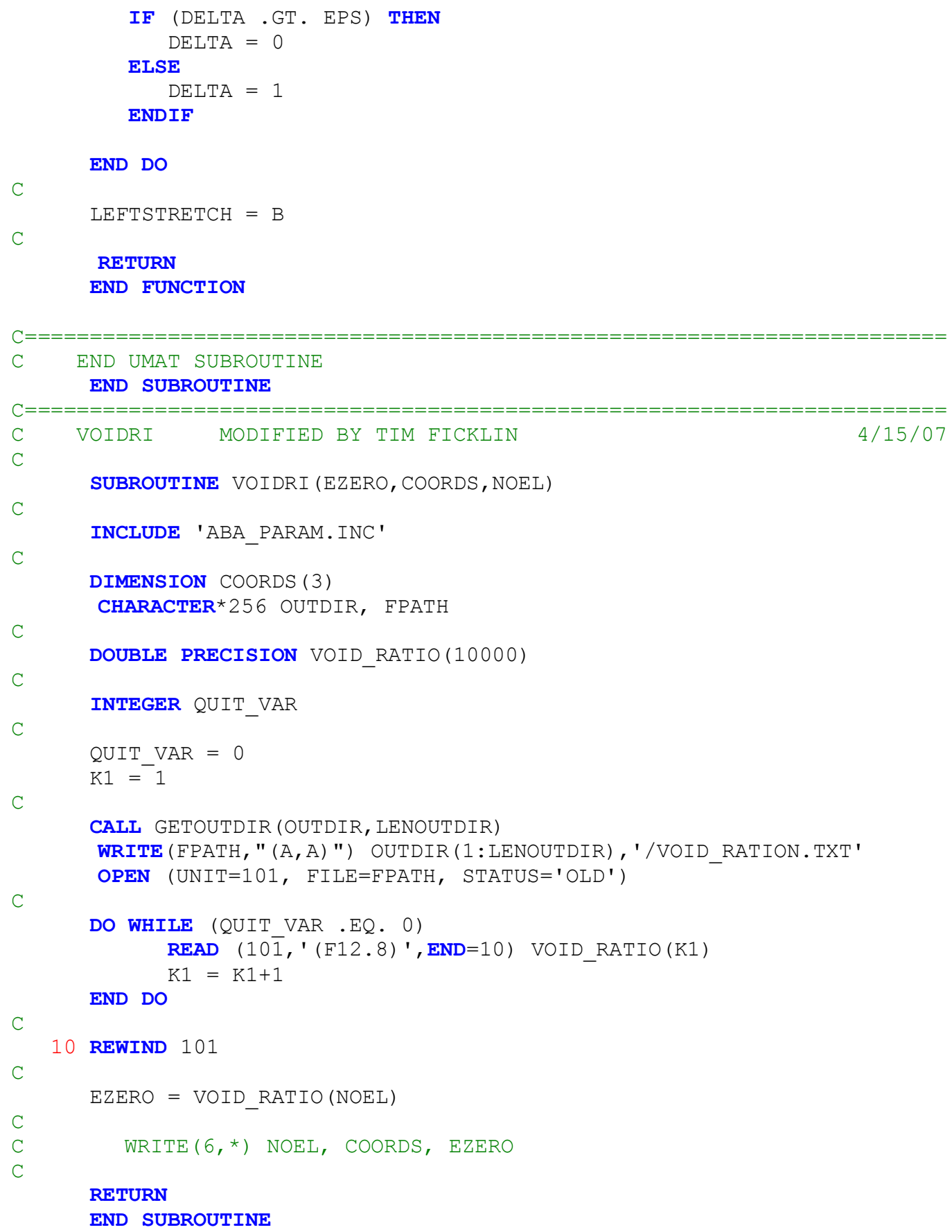




\section{E.1.2 DPF}

This section contains the DPF.for code (derived then pushed-forward), including the UMAT subroutine, as used in this work. DPF.for also contains the following functions internal to the UMAT as in the PFD code: DETERMINANT(), MAT-

INV(), and LEFTSTRETCH. These have been omitted here for space considerations. DPF.for also contains the VOIDRI subroutine (omitted here) after the UMAT subroutine as in the PFD code.

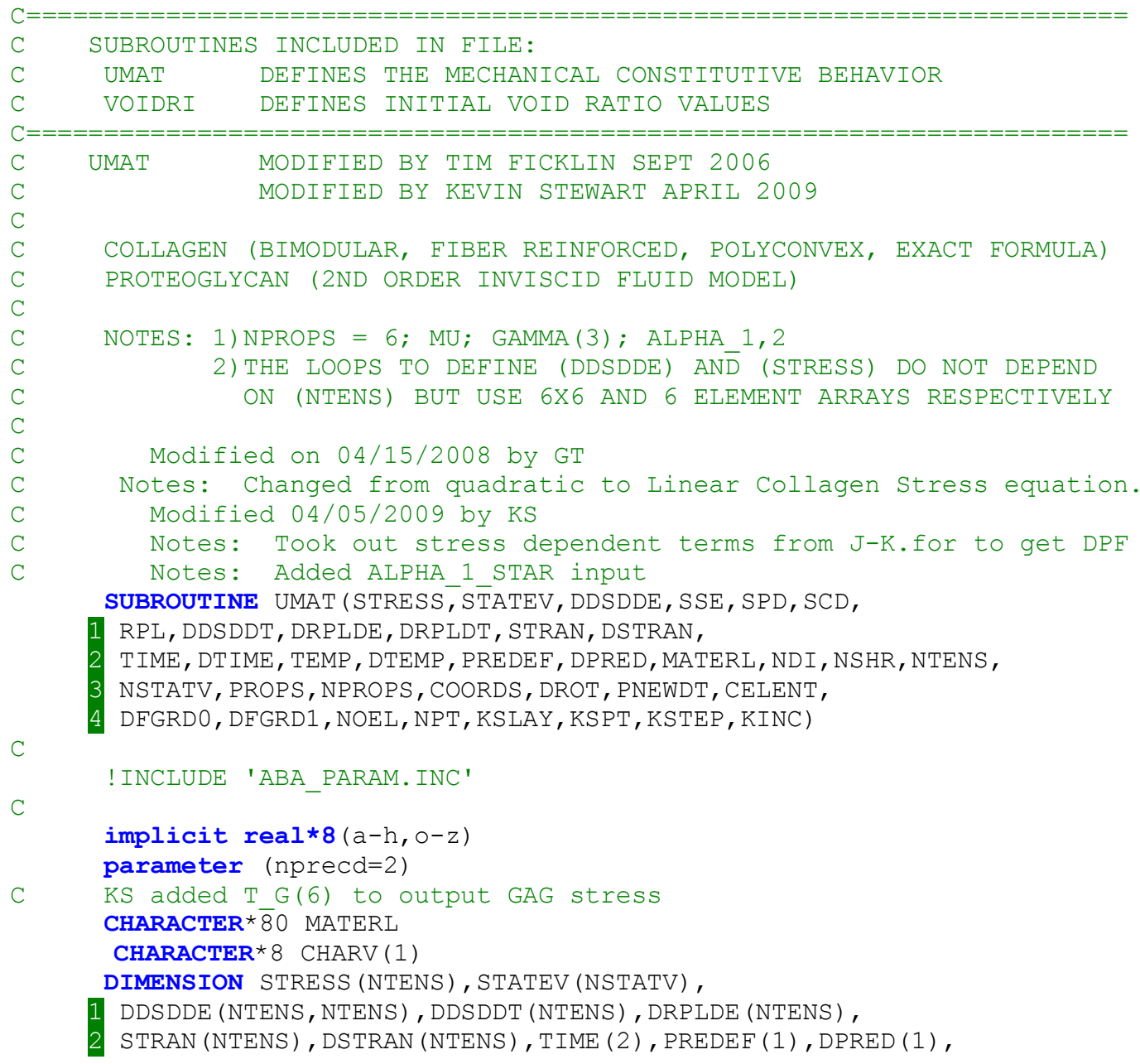


PROPS (NPROPS), COORDS (3), DROT $(3,3), \operatorname{DFGRDO}(3,3), \operatorname{DFGRD} 1(3,3)$,

FO_C $(3,3), F O \_P(3,3), \operatorname{STIFFCPF}(N T E N S, \operatorname{NTENS}), \operatorname{STIFFP}(N T E N S, N T E N S)$,

STIFFFTOT (NTENS, NTENS), STIFFCT1 (NTENS, NTENS), T GAG (NTENS, NTENS),

STIFFPT2 (NTENS, NTENS), STIFFPT1 (NTENS, NTENS), T_COL (NTENS, NTENS),

IDENT $(3,3)$, STRANB (NTENS), PSTRESS (NTENS), STRANC (NTENS),

$\operatorname{FC}(3,3), \operatorname{FP}(3,3), \operatorname{VP}(3,3), \operatorname{VP} \operatorname{INV}(3,3), \mathrm{T}(6), \mathrm{T} \in \mathrm{G}(6), \mathrm{CC}(3,3)$,

$\operatorname{BP}(3,3), \operatorname{BC}(3,3), \operatorname{VC}(3,3), \overline{\operatorname{VC}} \operatorname{INV}(3,3), \operatorname{RC}(\overline{3}, 3), \operatorname{INDEX}(2,6)$,

$1 \operatorname{COEFF}(3,3), \operatorname{PHI}(3,3), \operatorname{STRETCH}(3,3), M(3,3,3,3)$,

C

C

C

C

$\mathrm{C}$

C

C

C

C

$\mathrm{C}$

C

INTEGER I, J, K, L, INDEX

REAL MU, COEFF, PHI, ALPHA_1, ALPHA_2, RHOO_P, M

DOUBLE PRECISION DDSDDE, BC, STIFFCT1, STIFFP, BP, DET VC,

$2 \mathrm{VC}, \mathrm{FC}, \mathrm{VP}, \mathrm{FP}, \mathrm{DET} V \mathrm{VP}, \mathrm{VP} I N V, \mathrm{VC} I N V, \mathrm{DET} F C, \mathrm{RC}, \mathrm{CC}$, 3 STRETCH, DET_DFGRD1, DET_FOP, STIFFCPF, STIFFPT1, 4 STIFFCTOT

\section{ASSIGN RESIDUAL DEFORMATIONS TO PROPER VARIABLES}

FO_C $(1,1)=\operatorname{STATEV}(1)$

$\mathrm{FO}^{-} \mathrm{C}(2,2)=\operatorname{STATEV}(2)$

$\mathrm{FO}^{-} \mathrm{C}(3,3)=\operatorname{STATEV}(3)$

$\mathrm{FO}^{-} \mathrm{C}(1,2)=\operatorname{STATEV}(4)$

$\mathrm{FO}^{-} \mathrm{C}(1,3)=\operatorname{STATEV}(5)$

$\mathrm{FO}^{-} \mathrm{C}(2,1)=\operatorname{STATEV}(6)$

$\mathrm{FO}^{-} \mathrm{C}(2,3)=\operatorname{STATEV}(7)$

$\mathrm{FO}^{-} \mathrm{C}(3,1)=\operatorname{STATEV}(8)$

$\mathrm{FO}_{-}^{-} \mathrm{C}(3,2)=\operatorname{STATEV}(9)$

$\mathrm{FO} P(1,1)=\operatorname{STATEV}(10)$

FO_P $(2,2)=\operatorname{STATEV}(11)$

$\mathrm{FO}^{-} \mathrm{P}(3,3)=\operatorname{STATEV}(12)$

$\mathrm{FO}_{-} \mathrm{P}(1,2)=\operatorname{STATEV}(13)$

$\mathrm{FO}^{-} \mathrm{P}(1,3)=\operatorname{STATEV}(14)$

$\mathrm{FO}^{-} \mathrm{P}(2,1)=\operatorname{STATEV}(15)$

$\mathrm{FO}^{-} \mathrm{P}(2,3)=\operatorname{STATEV}(16)$

$\mathrm{FO}^{-} \mathrm{P}(3,1)=\operatorname{STATEV}(17)$

$\mathrm{FO}_{-}^{-} \mathrm{P}(3,2)=\operatorname{STATEV}(18)$

COLLAGEN (COL) CONSTITUENT - TENSORS AND DETERMINANTS

$\mathrm{FC}=$ MATMUL (DFGRD1, FO C) !TOTAL COL DEFORMATION GRADIENT, FC

$\mathrm{BC}=\operatorname{MATMUL}(\mathrm{FC}, \mathrm{TRANSPO} \mathrm{SE}(\mathrm{FC}))$ !LEFT CAUCHY-GREEN STRESS, BC

$\mathrm{VC}=$ LEFTSTRETCH (BC) !LEFT STRETCH TENSOR, VC

$\mathrm{CC}=$ MATMUL (TRANSPOSE (FC), FC) !RIGHT CAUCHY-GREEN STRESS, CC

VC_INV = MATINV (VC) !INVERSE OF LEFT STRETCH, VC_INV

$\mathrm{RC}^{-}=$MATMUL $\left(\mathrm{VC} \_\right.$INV, FC) $\quad$ ! ROTATION TENSOR, RC

DET_FC $=$ DETERMINANT $($ FC) !COLLAGEN JACOBIAN

DET_ $\bar{V}$ V $=$ DETERMINANT $(\mathrm{VC}) \quad$ ! DETERMINANT OF LEFT STRETCH

PROTEOGLYCAN (PG) CONSTITUENT - TENSORS AND DETERMINANTS

$\mathrm{FP}=$ MATMUL (DFGRD1,FO_P ) !TOTAL PG DEFORMATION GRADIENT, FP 


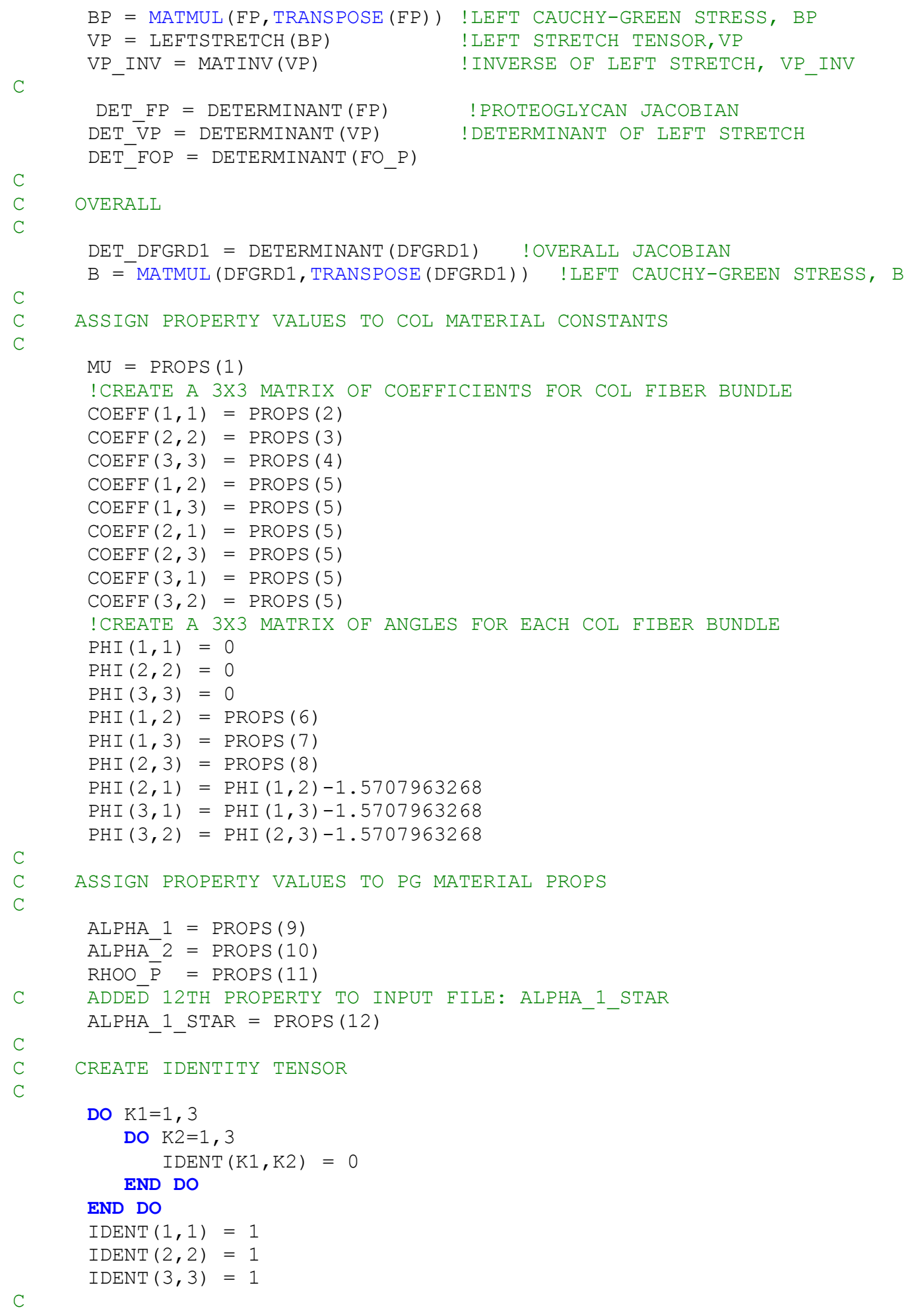




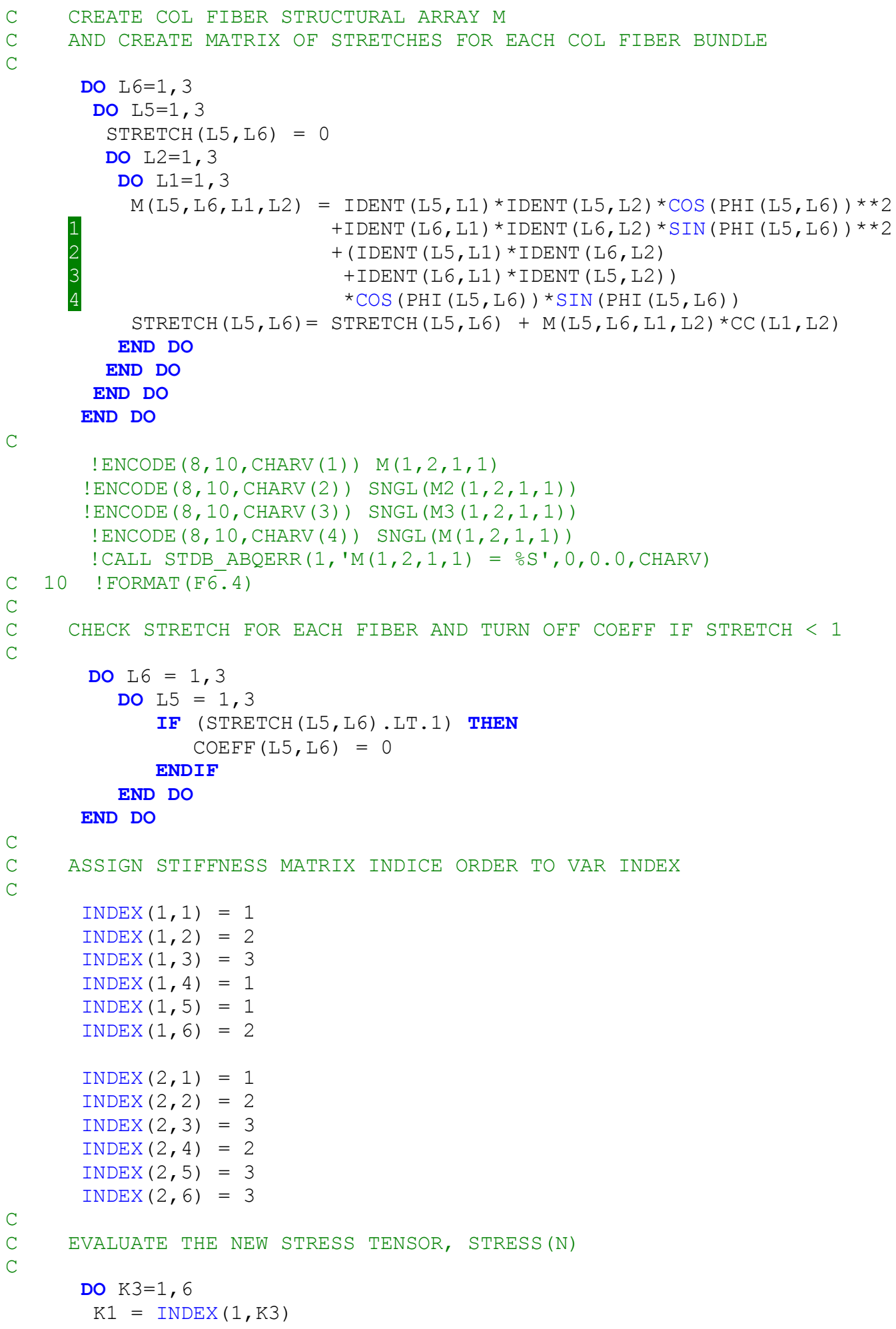




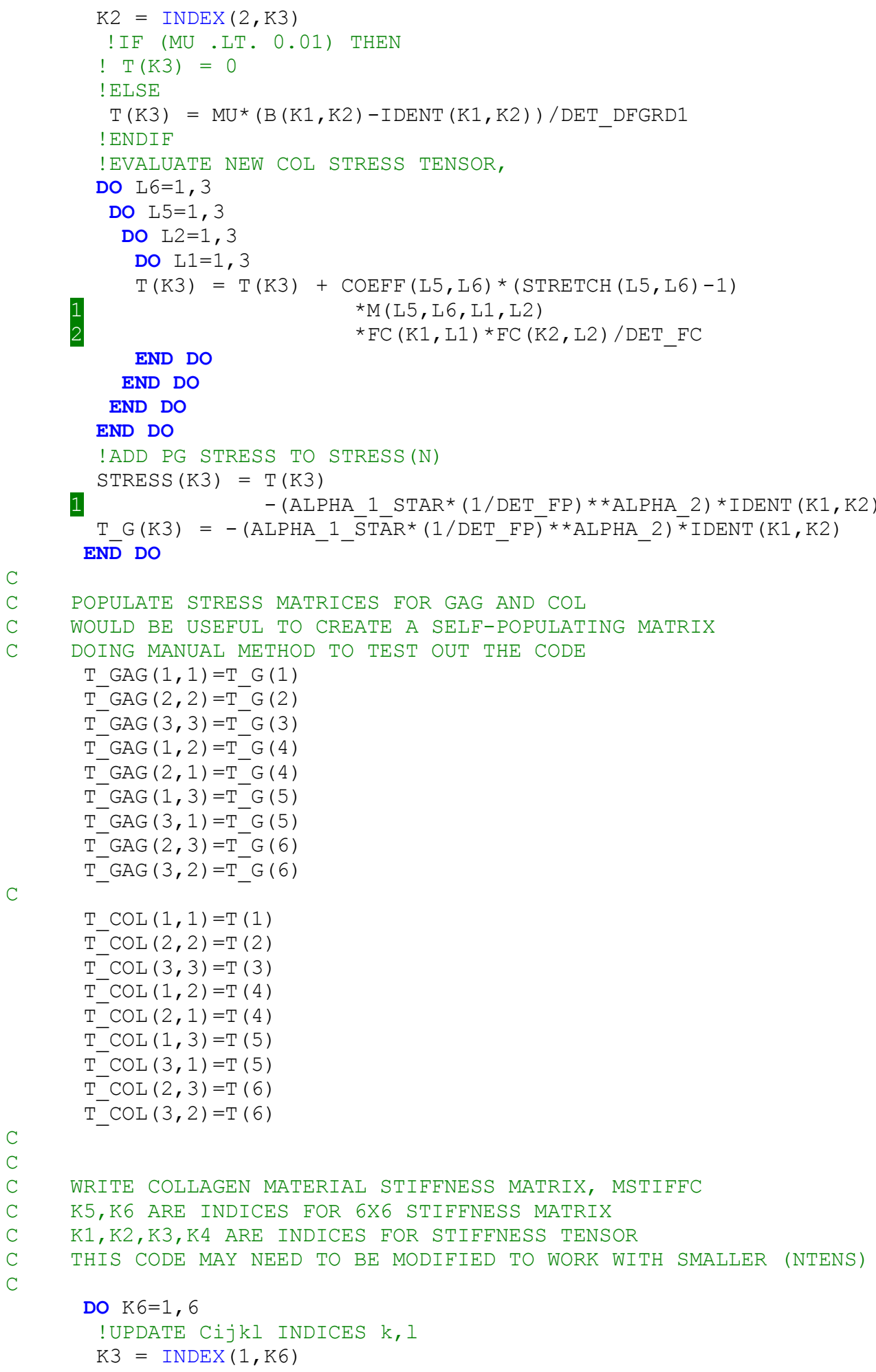




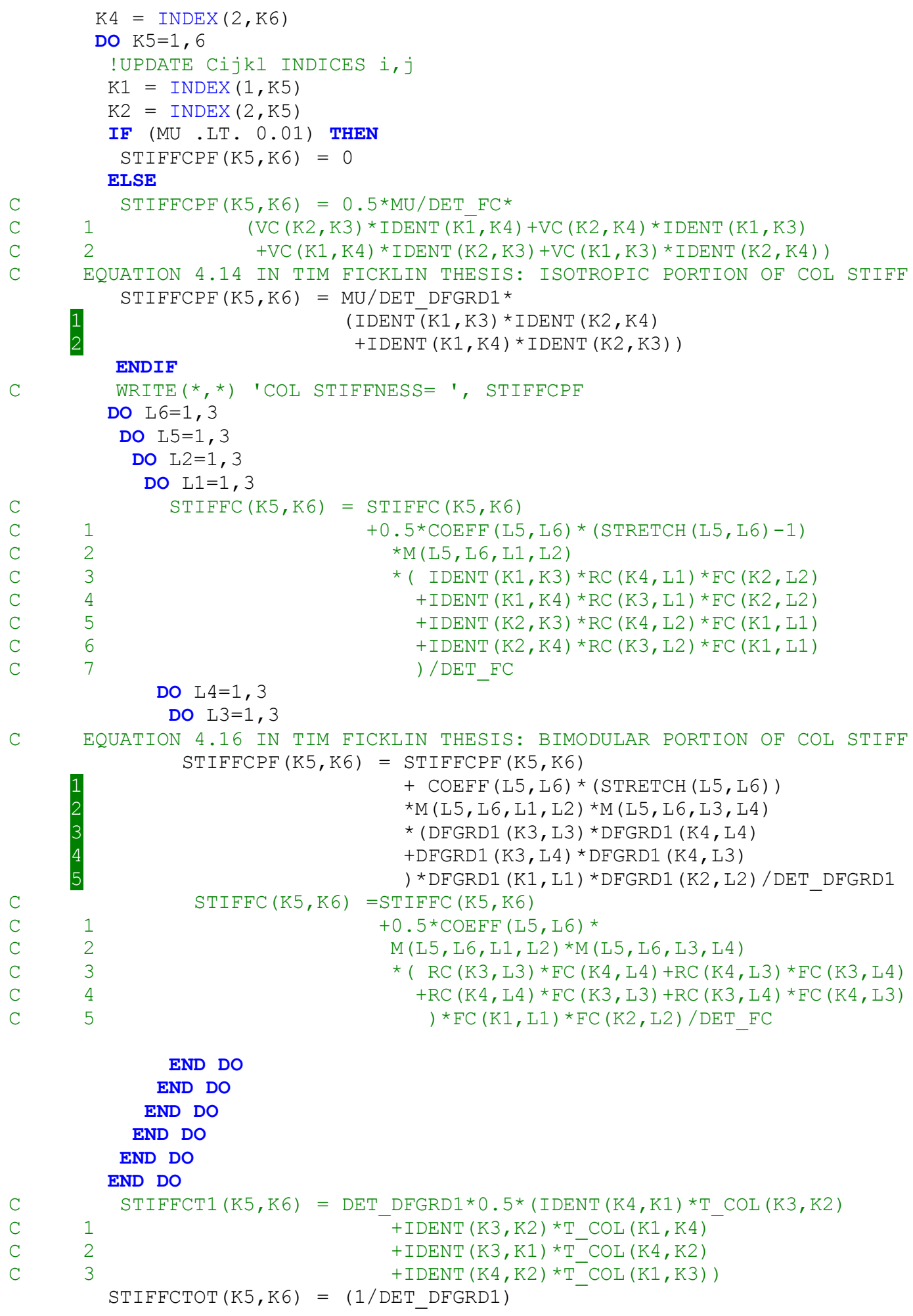

112 


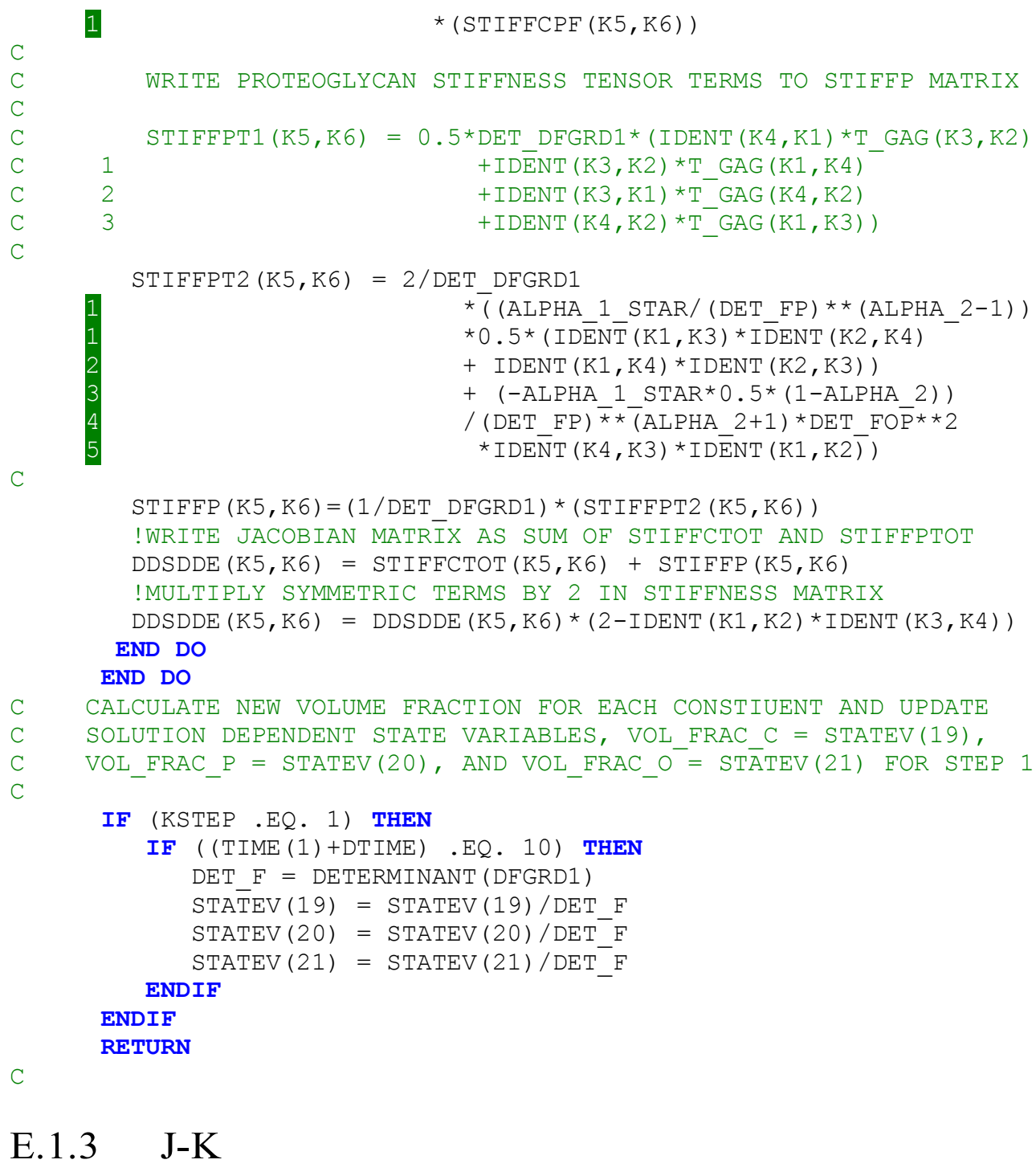

This section contains the J-K. for code (Jaumann-Kirchoff Method), including the UMAT and VOIDRI subroutines, as used in this work. J-K.for also contains the following functions internal to the UMAT as in the PFD code: DETERMINANT(), MATINV(), and LEFTSTRETCH. These have been omitted here for 


\section{space considerations. J-K.for also contains the VOIDRI subroutine (omitted here)}

\section{after the UMAT subroutine as in the PFD code.}

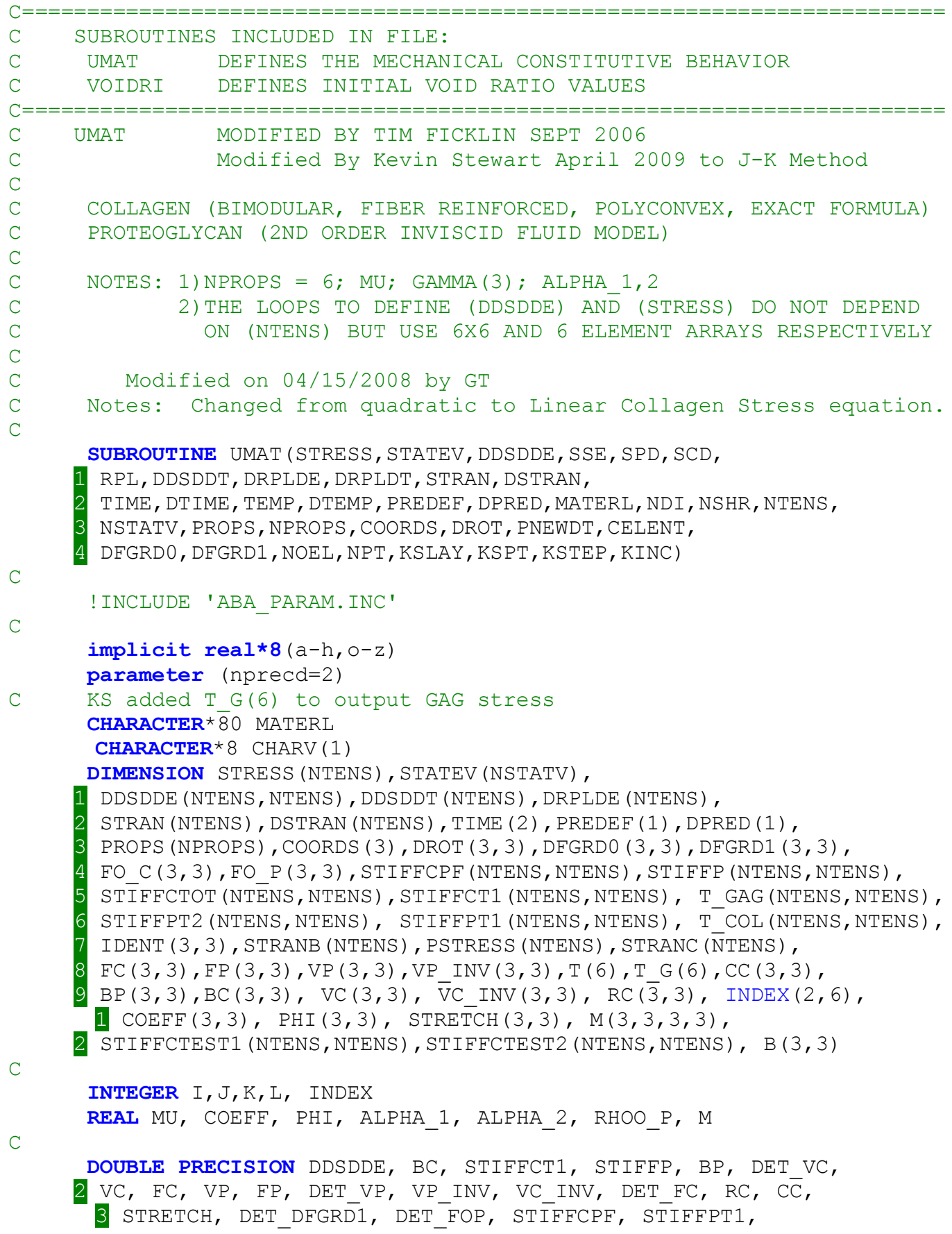


STIFFCTOT

$\mathrm{C}$

FO_C $(1,1)=\operatorname{STATEV}(1)$

$\mathrm{FO}^{-} \mathrm{C}(2,2)=\operatorname{STATEV}(2)$

$\mathrm{FO} C(3,3)=\operatorname{STATEV}(3)$

$\mathrm{FO} C(1,2)=\operatorname{STATEV}(4)$

$\mathrm{FO}^{-} \mathrm{C}(1,3)=\operatorname{STATEV}(5)$

$\mathrm{FO}^{-} \mathrm{C}(2,1)=\operatorname{STATEV}(6)$

$\mathrm{FO}^{-} \mathrm{C}(2,3)=\operatorname{STATEV}(7)$

$\mathrm{FO}^{-} \mathrm{C}(3,1)=\operatorname{STATEV}(8)$

FO_C $(3,2)=\operatorname{STATEV}(9)$

C

FO P $(1,1)=\operatorname{STATEV}(10)$

FO $P(2,2)=$ STATEV $(11)$

$\mathrm{FO}^{-} \mathrm{P}(3,3)=\operatorname{STATEV}(12)$

$\mathrm{FO}^{-} \mathrm{P}(1,2)=\operatorname{STATEV}(13)$

$\mathrm{FO}^{-} \mathrm{P}(1,3)=\operatorname{STATEV}(14)$

$\mathrm{FO}^{-} \mathrm{P}(2,1)=\operatorname{STATEV}(15)$

FO_P $(2,3)=\operatorname{STATEV}(16)$

$\mathrm{FO}^{-} \mathrm{P}(3,1)=\operatorname{STATEV}(17)$

C

FO_P $(3,2)=$ STATEV $(18)$

COLLAGEN (COL) CONSTITUENT - TENSORS AND DETERMINANTS

$\mathrm{FC}=$ MATMUL (DFGRD1, FO C) !TOTAL COL DEFORMATION GRADIENT, FC

$\mathrm{BC}=$ MATMUL (FC, TRANSPŌSE (FC)) !LEFT CAUCHY-GREEN STRESS, BC

$\mathrm{VC}=$ LEFTSTRETCH (BC) !LEFT STRETCH TENSOR, VC

$\mathrm{CC}=$ MATMUL (TRANSPOSE (FC), FC) !RIGHT CAUCHY-GREEN STRESS, CC

VC_INV = MATINV (VC) !INVERSE OF LEET STRETCH, VC_INV

$\mathrm{RC}^{-}=$MATMUL $($VC_INV, FC) !ROTATION TENSOR, RC

C

DET $F C=$ DETERMINANT $(F C) \quad$ !COLLAGEN JACOBIAN

DET $\overline{\mathrm{V} C}=$ DETERMINANT (VC) ! DETERMINANT OF LEFT STRETCH

$\mathrm{C}$

$\mathrm{C}$

PROTEOGLYCAN (PG) CONSTITUENT - TENSORS AND DETERMINANTS

$\mathrm{FP}=\mathrm{MATMUL}\left(\mathrm{DFGRD1}, \mathrm{FO} \mathrm{P}_{-}\right)$

!TOTAL PG DEFORMATION GRADIENT, FP

$\mathrm{BP}=$ MATMUL (FP, TRANSPŌSE (FP)) !LEFT CAUCHY-GREEN STRESS, BP

$\mathrm{VP}=\operatorname{LEFTSTRETCH}(\mathrm{BP})$

!LEFT STRETCH TENSOR, VP

VP_INV $=\operatorname{MATINV}(\mathrm{VP})$

! INVERSE OF LEFT STRETCH, VP_INV

C

DET_FP $=$ DETERMINANT $($ FP $) \quad$ ! PROTEOGLYCAN JACOBIAN

$\mathrm{DET} \overline{\mathrm{VP}}=$ DETERMINANT $(\mathrm{VP})$

!DETERMINANT OF LEFT STRETCH

$\mathrm{DET}^{-} \mathrm{FOP}=\operatorname{DETERMINANT}(\mathrm{FO} P)$

OVERALL

DET_DFGRD1 = DETERMINANT (DFGRD1) !OVERALL JACOBIAN

$\mathrm{B}=$ - MATMUL (DFGRD1, TRANSPOSE (DFGRD1)) !LEFT CAUCHY-GREEN STRESS, B

C

ASSIGN PROPERTY VALUES TO COL MATERIAL CONSTANTS

$\mathrm{MU}=\operatorname{PROPS}(1)$ 


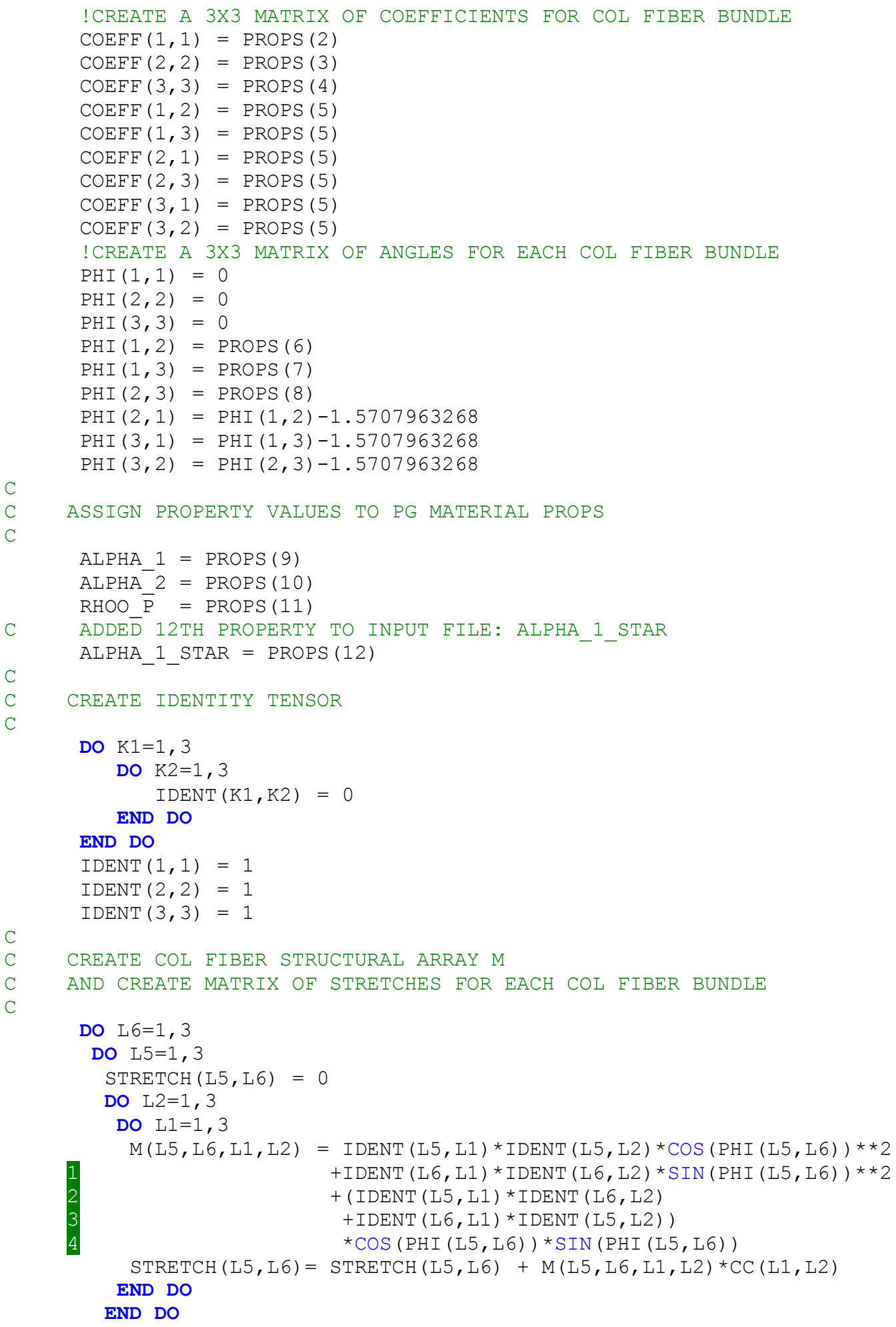




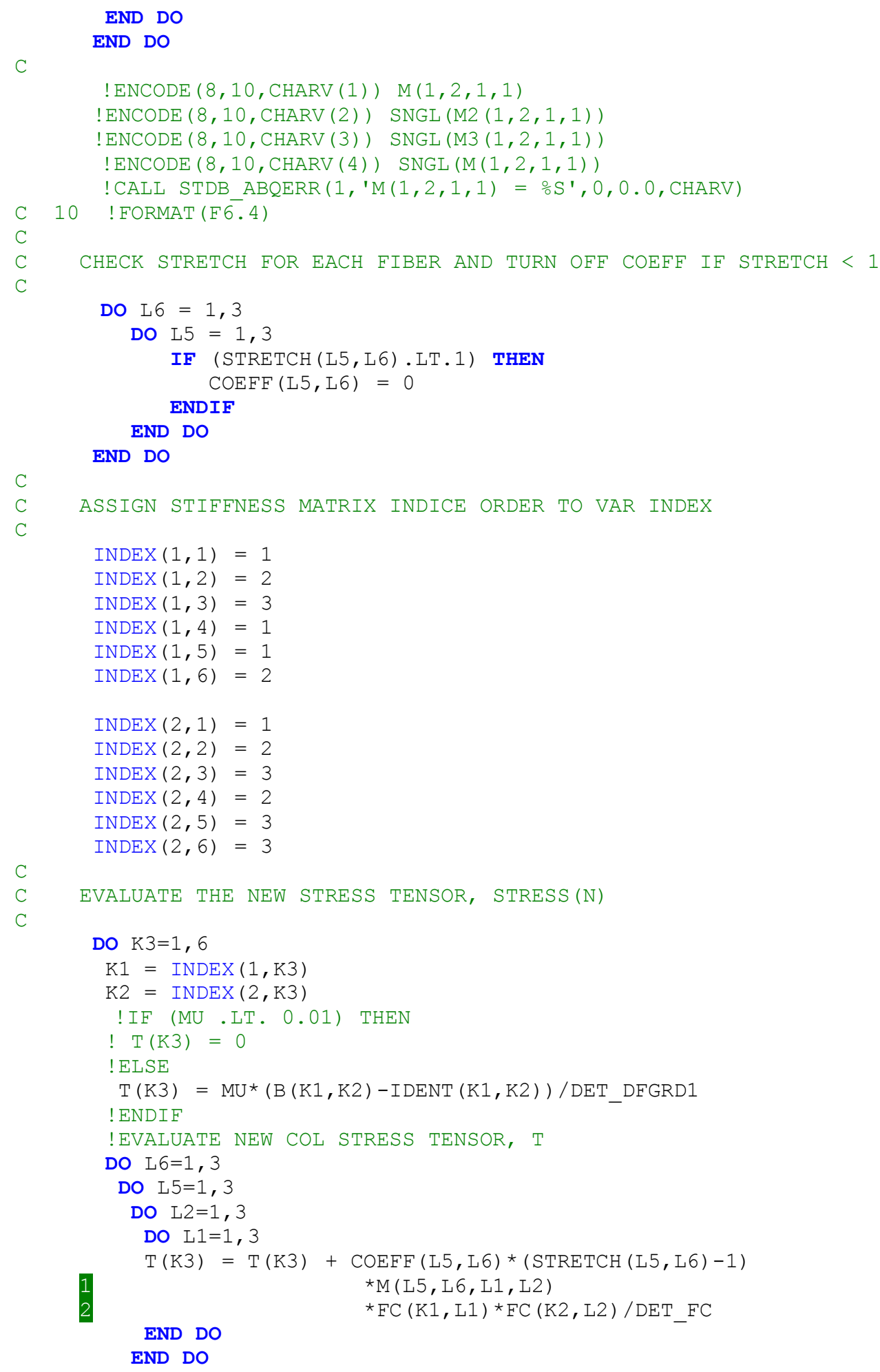




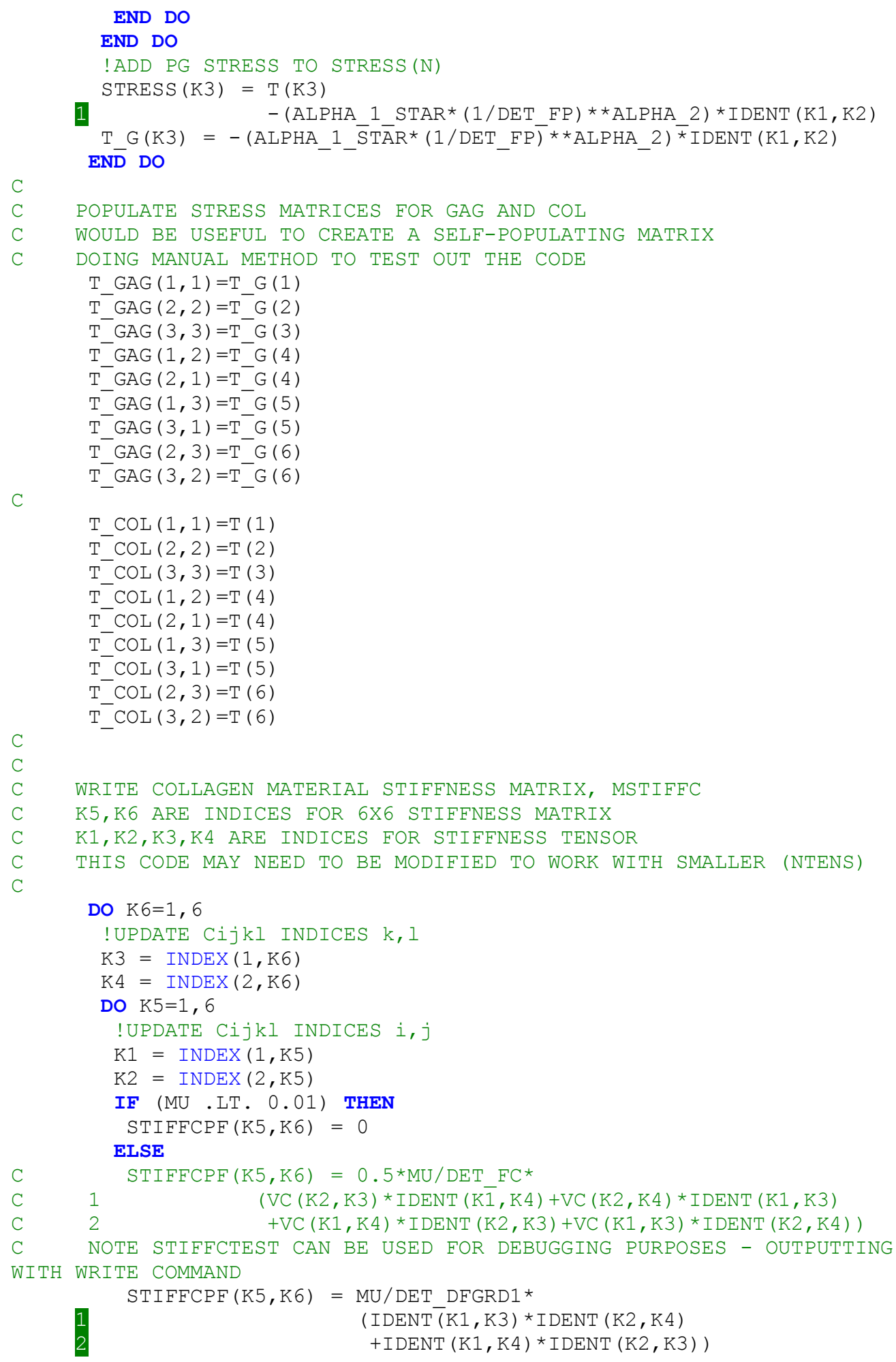




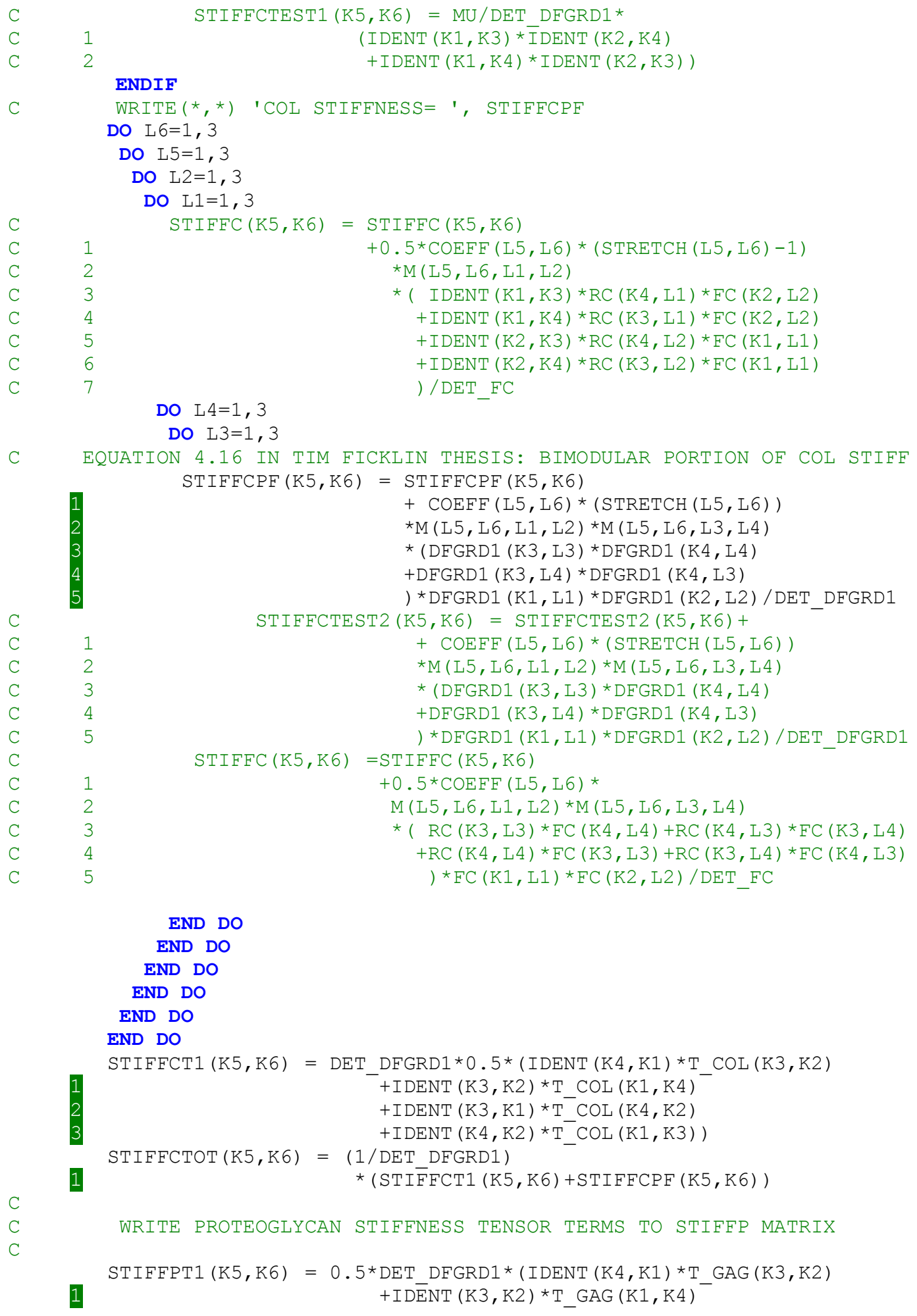




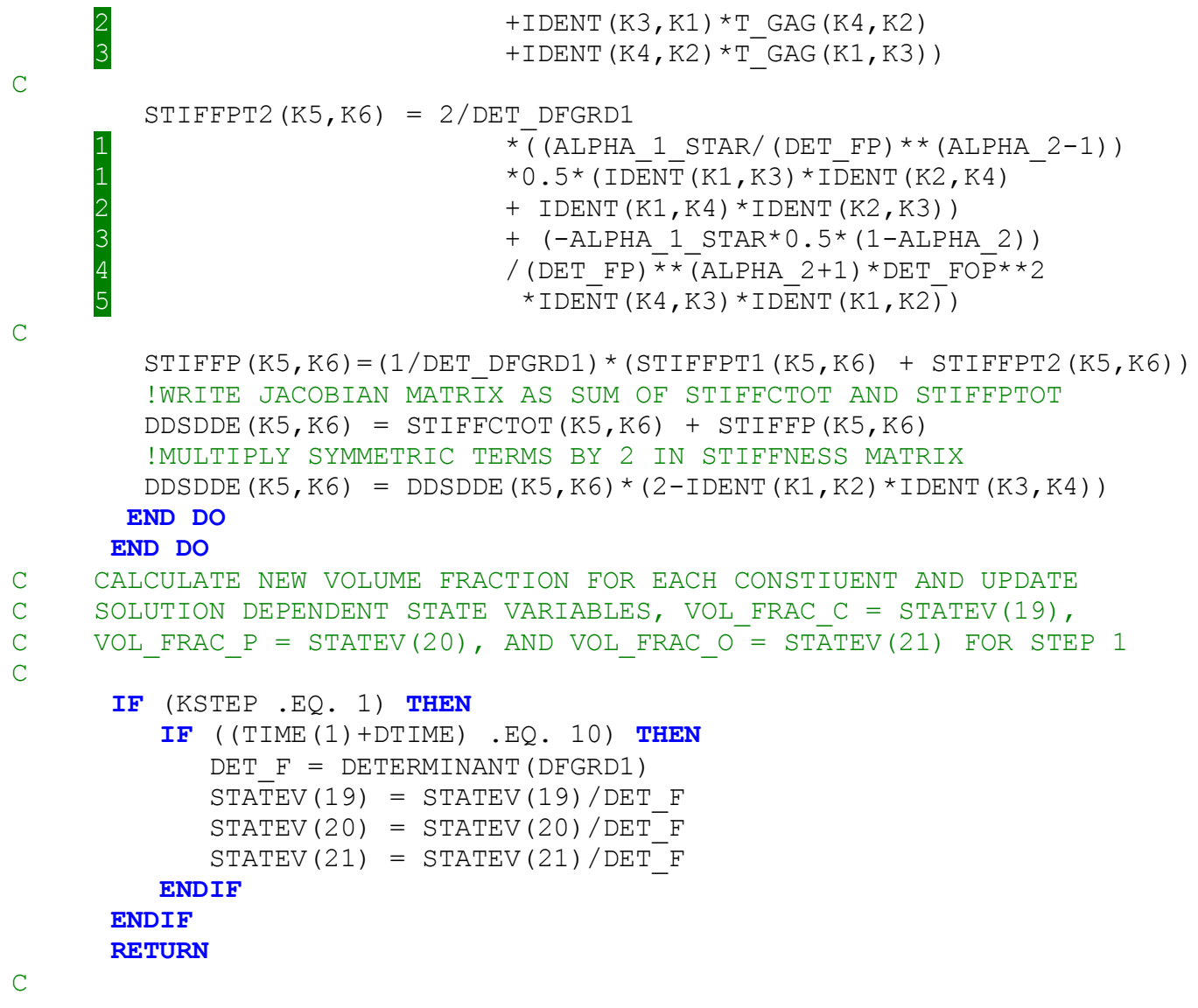

\section{E.2 ABAQUS Input Files}

\section{E.2.1 Uniaxial Tension Elastic}

The following is the ABAQUS input file for elastic uniaxial tension (UT_KSmodified_1EL_MD.inp) 1 element (C3D8) simulations used in this work. Note that the FCO and displacement values were for a particular run and may need to be modified for future runs.

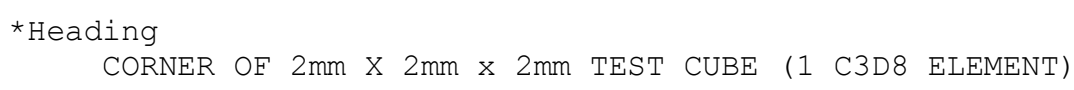




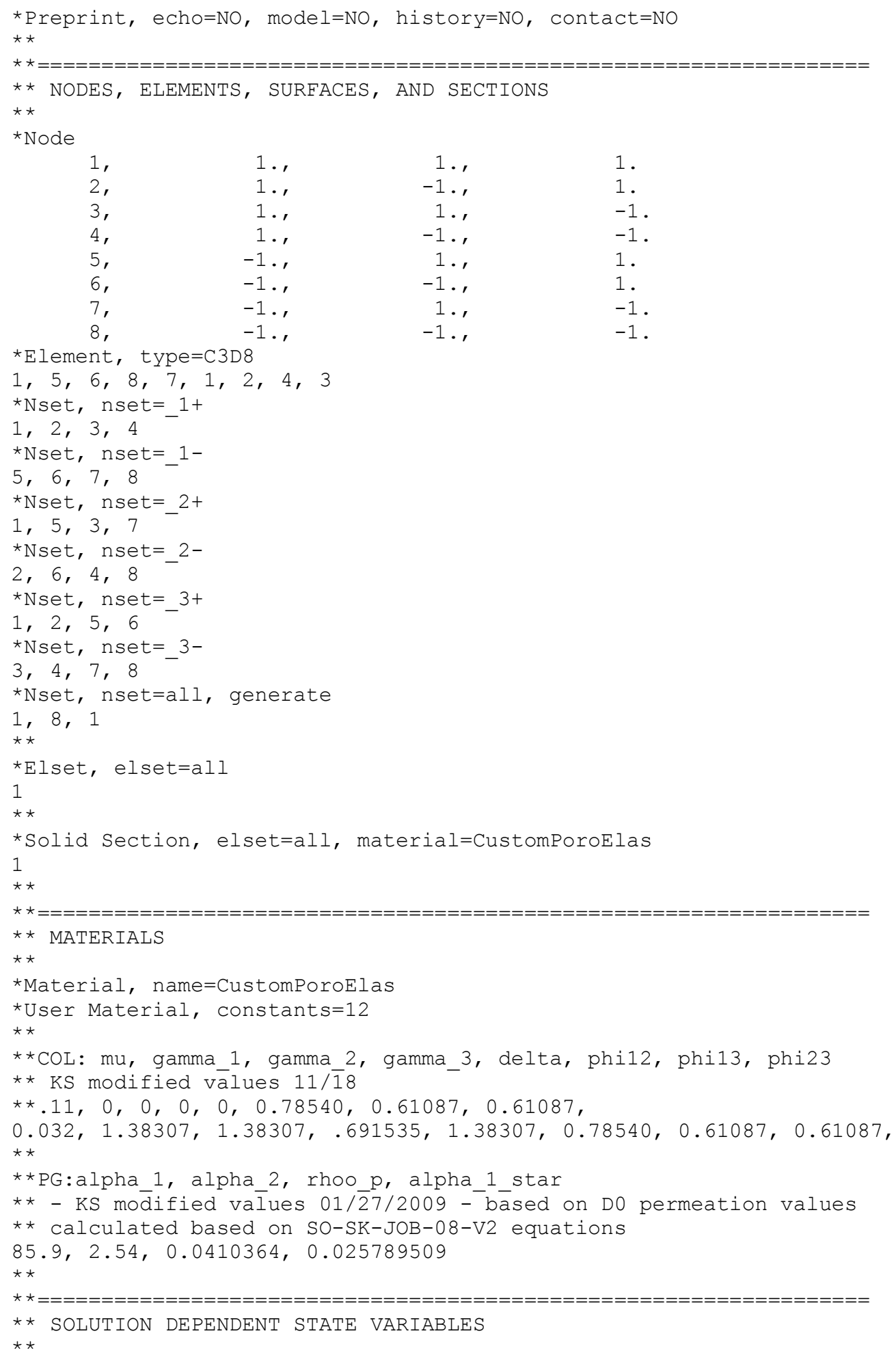




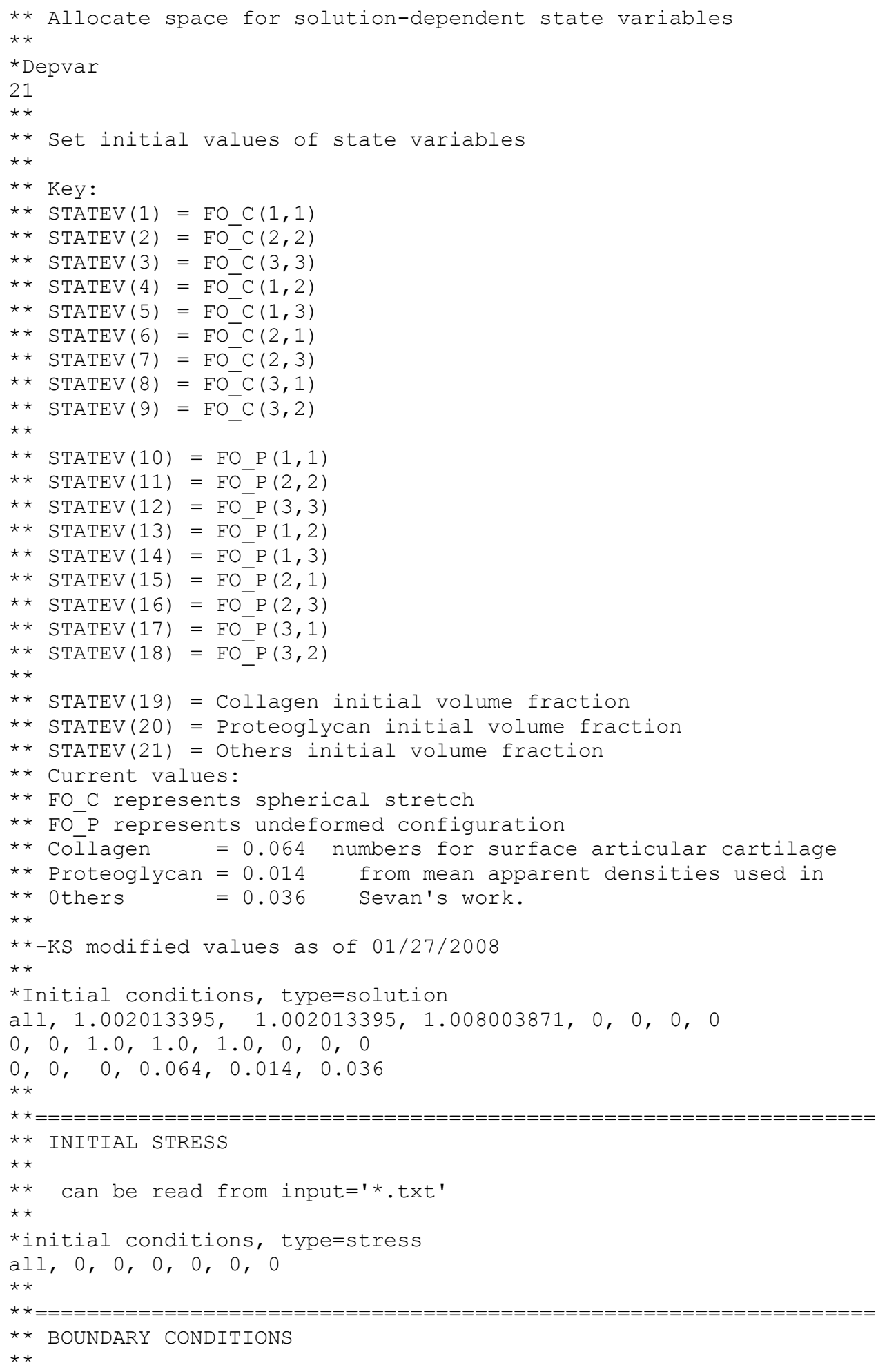




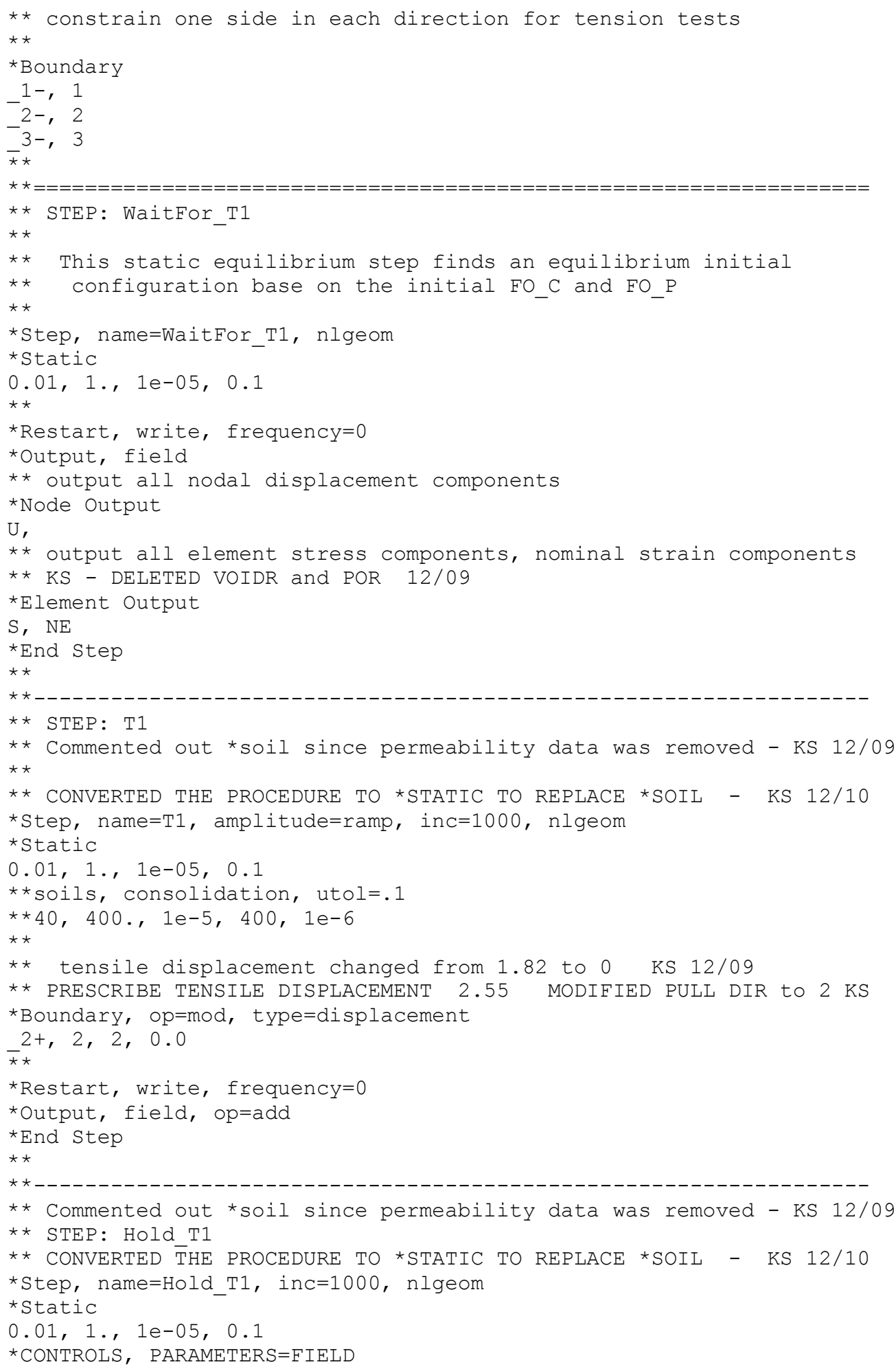




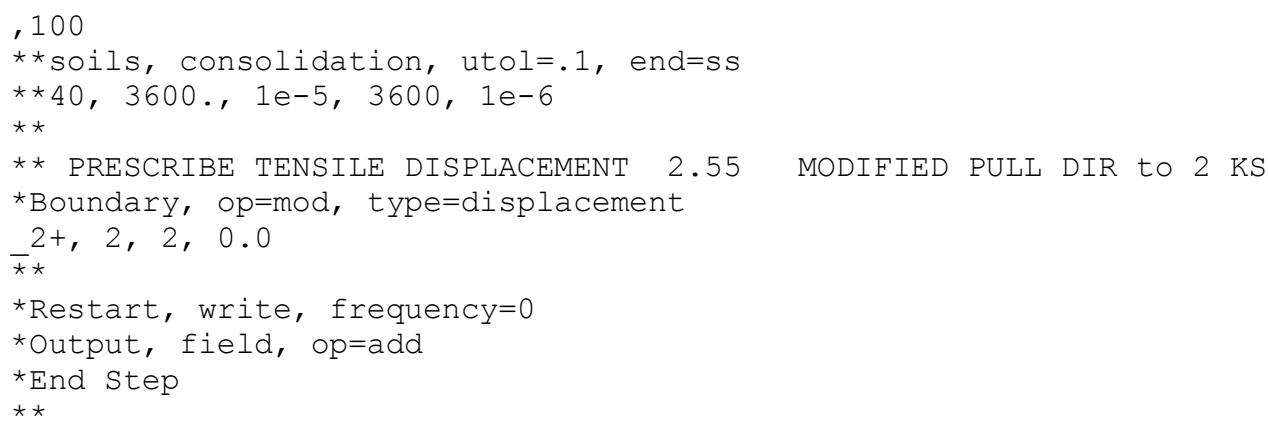

\section{E.2.2 Unconfined Compression Elastic}

The following is the ABAQUS input file for elastic unconfined compression (UCC_KSmodified_1EL_MD.inp) 1 element (C3D8) simulations used in this work. Note that the FCO and displacement values were for a particular run and may need to be modified for future runs. Many of the comments are the same as the input file for $\mathrm{CC}$ and have been omitted here.

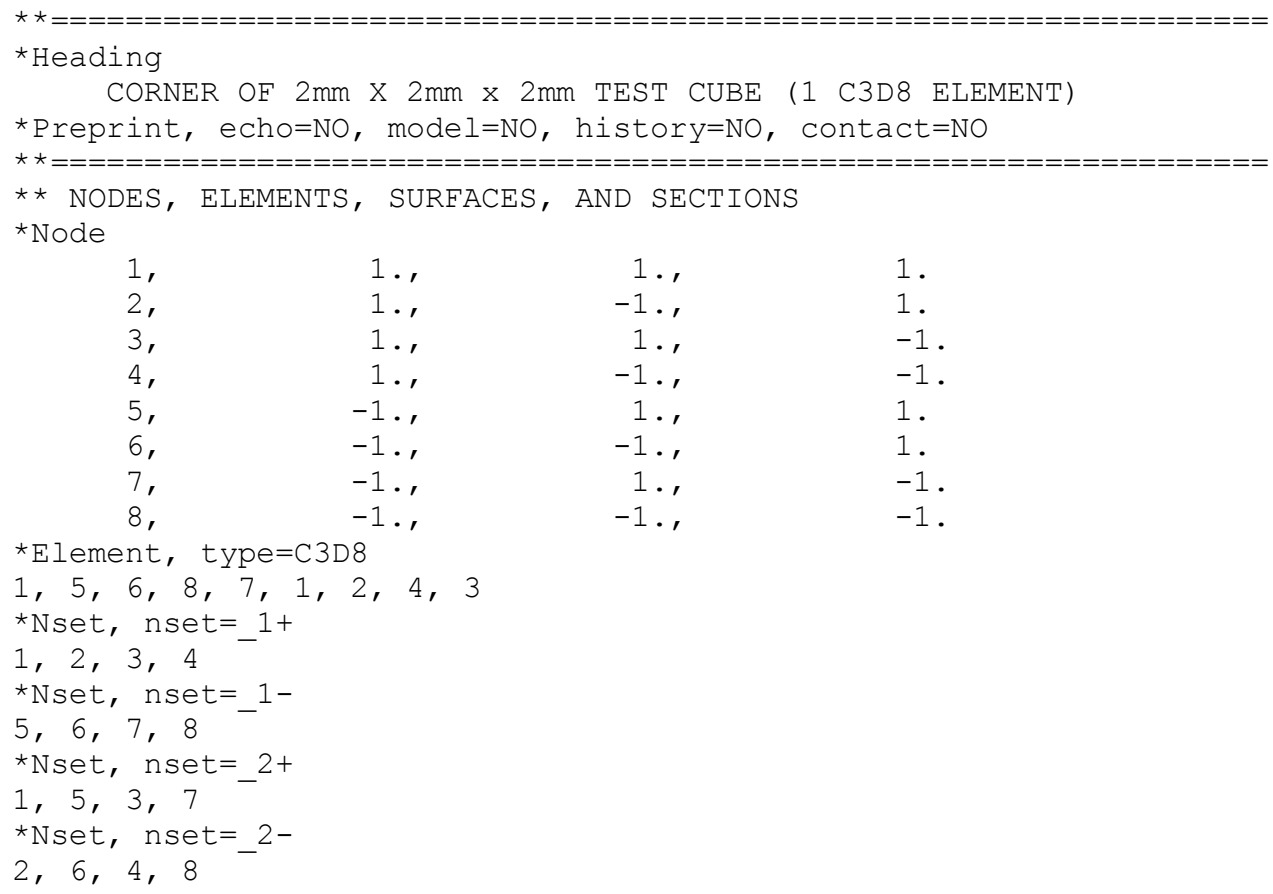




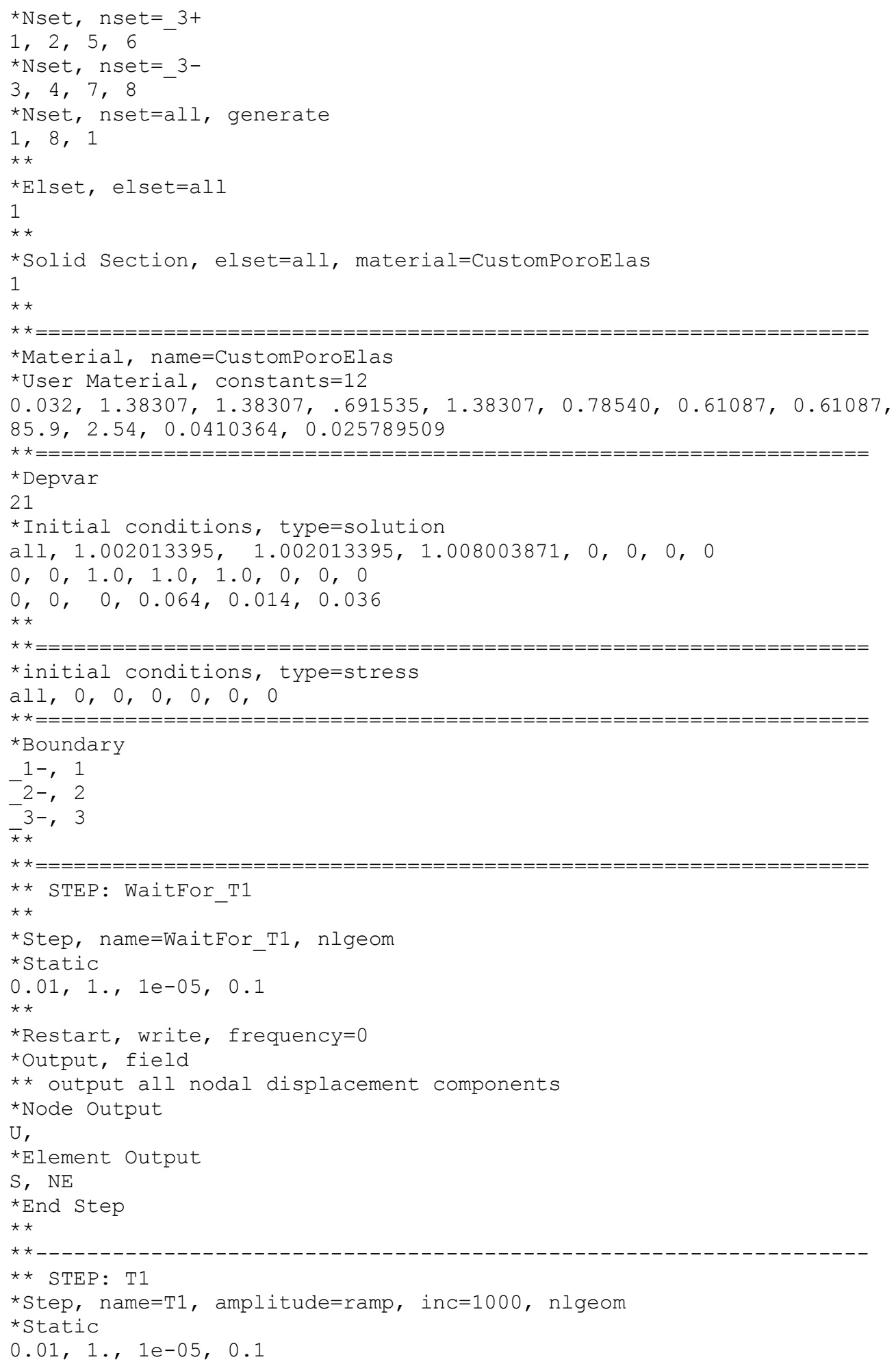




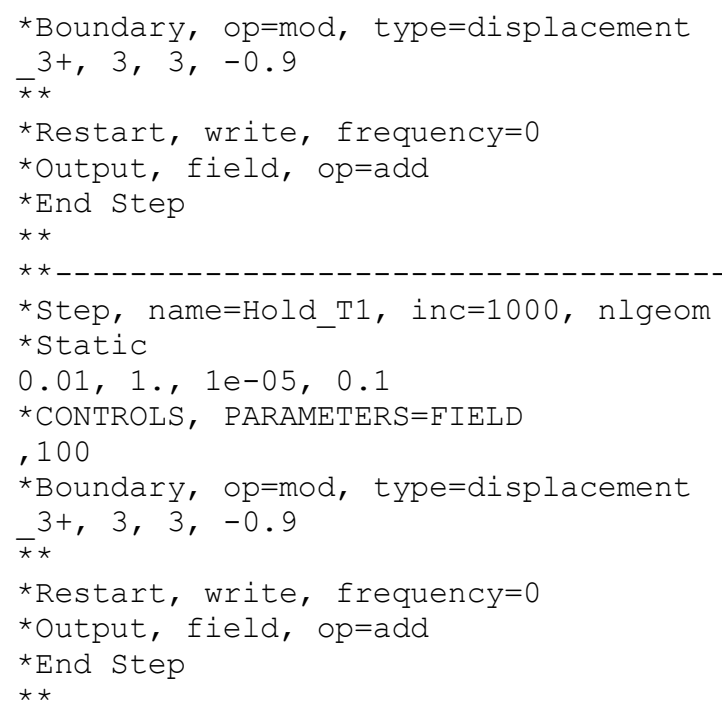

\section{E.2.3 Confined Compression Elastic}

The following is the ABAQUS input file for elastic confined compression (CC_KSmodified_1EL_MD.inp) 1 element (C3D8) simulations used in this work. Note that the FCO and displacement values were for a particular run and may need to be modified for future runs. Many of the comments are the same as the input file for $\mathrm{CC}$ and have been omitted here.

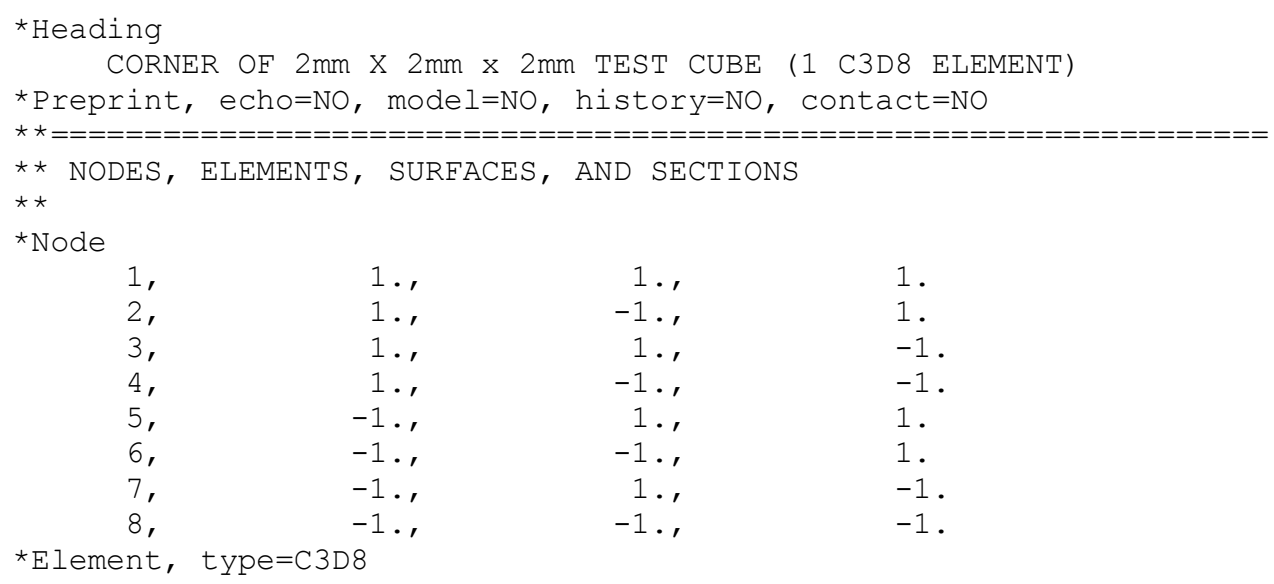

$\begin{array}{rrrr}1, & 1 ., & 1 ., & 1 . \\ 2, & 1 ., & -1 ., & 1 . \\ 3, & 1 ., & 1 ., & -1 . \\ 4, & 1 ., & -1 ., & -1 . \\ 5, & -1 ., & 1 ., & 1 . \\ 6, & -1 ., & -1 ., & 1 . \\ 7, & -1 ., & 1 ., & -1 . \\ 8, & -1 ., & -1 ., & -1 .\end{array}$




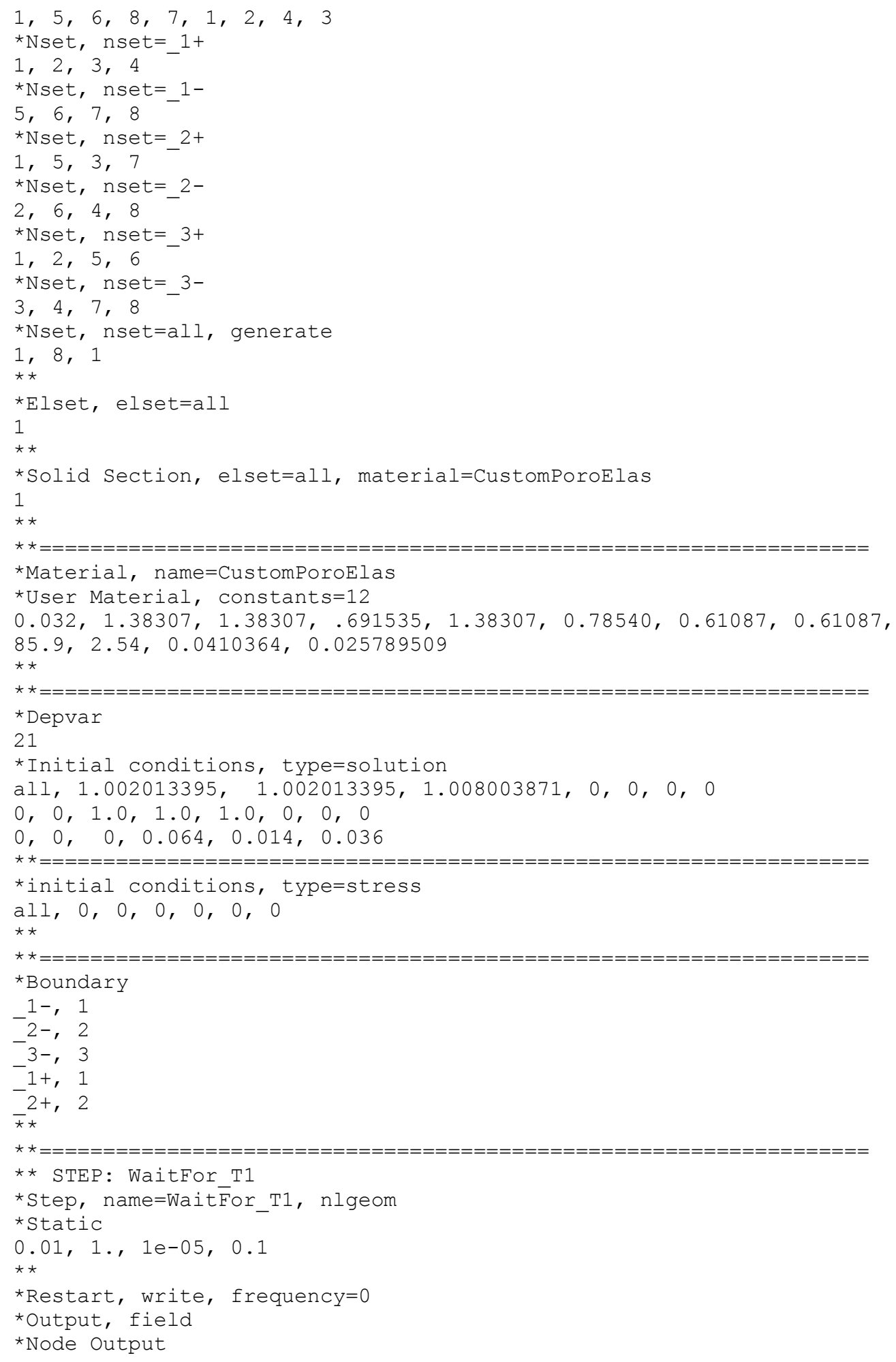




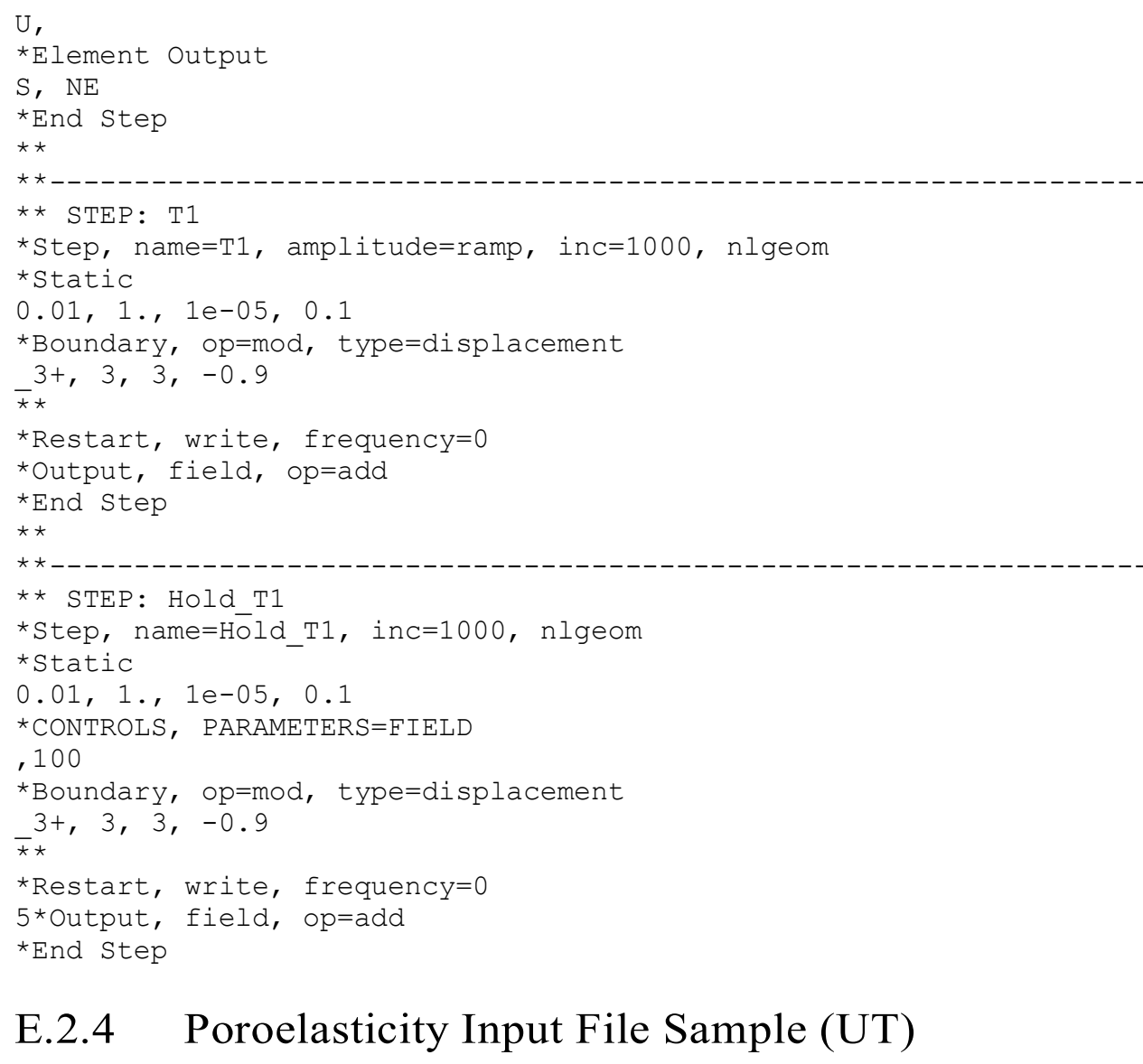

\section{E.2.4 Poroelasticity Input File Sample (UT)}

The following is the ABAQUS input file for poroelastic uniaxial tension (UT_KSmodified_1EL_MD_poro.inp) 1 element (C3D8P) simulations used in this work. Note that the FCO and displacement values were for a particular run and may need to be modified for future runs. This file differs from the elastic input files in that it contains a permeability lookup table, void ratio calculations, and pore pressure boundary conditions.

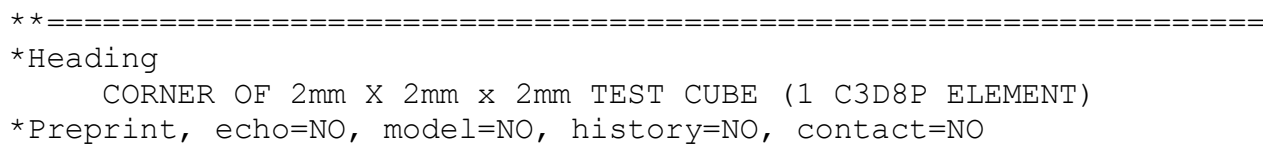




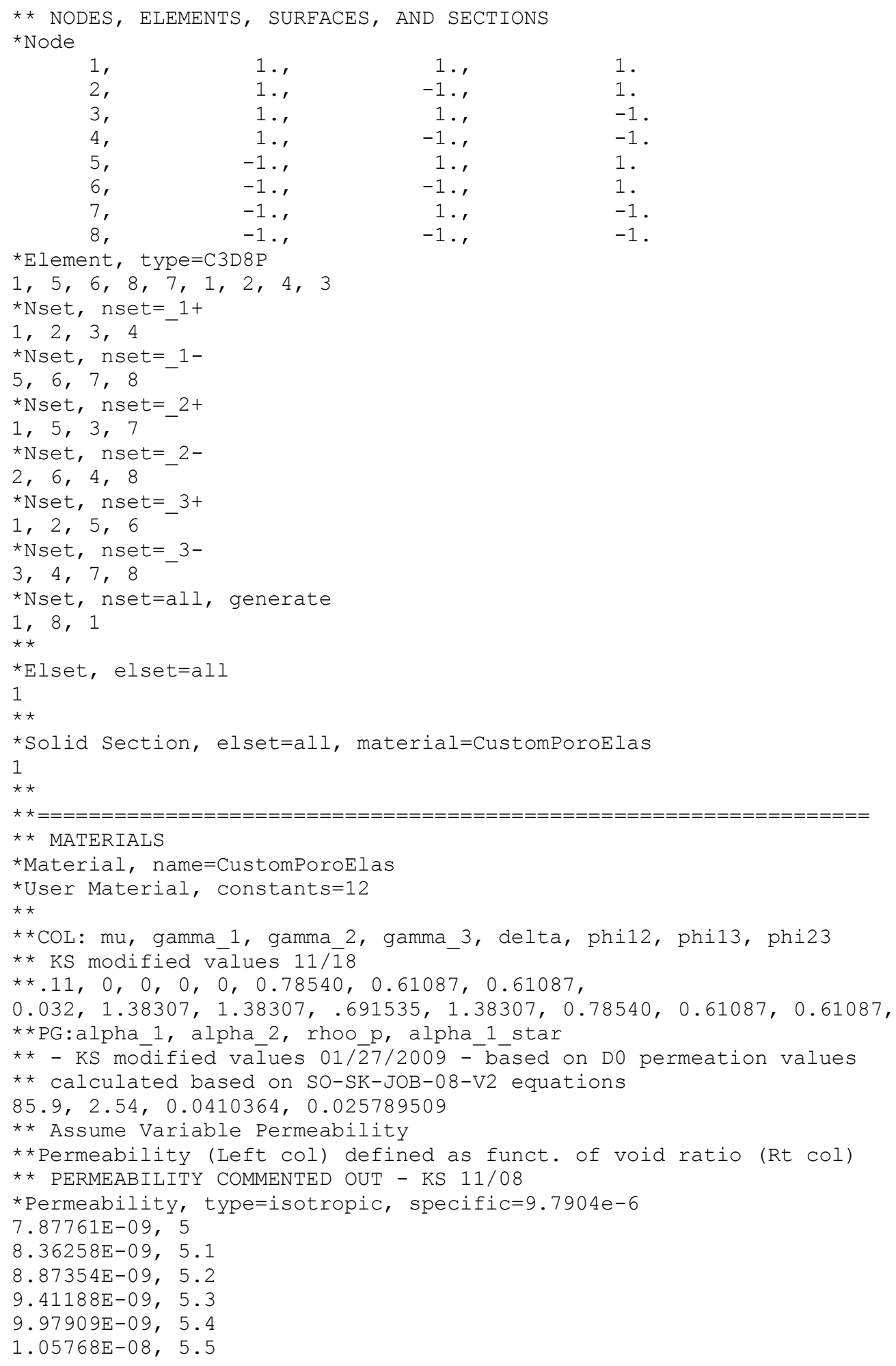




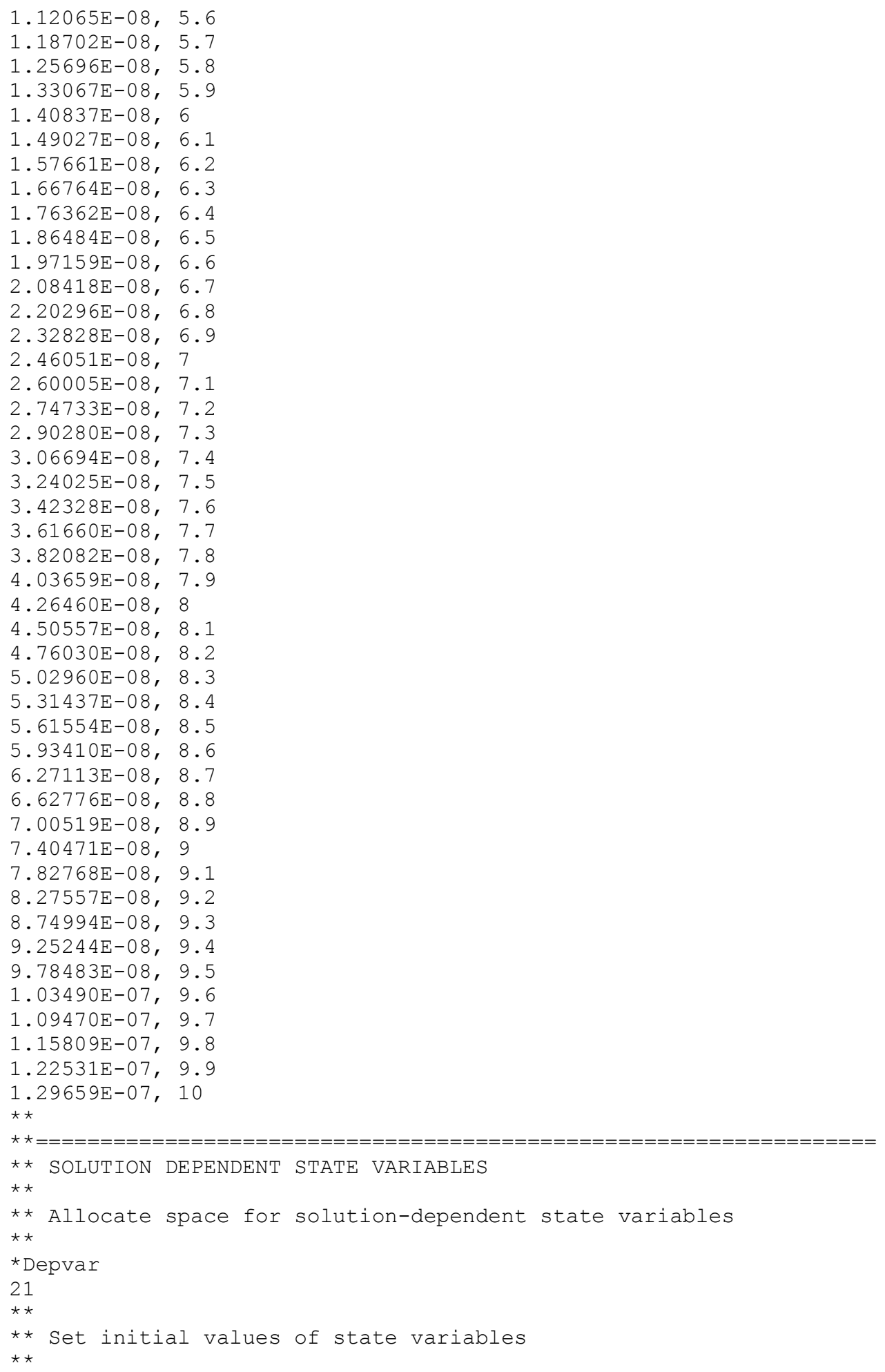




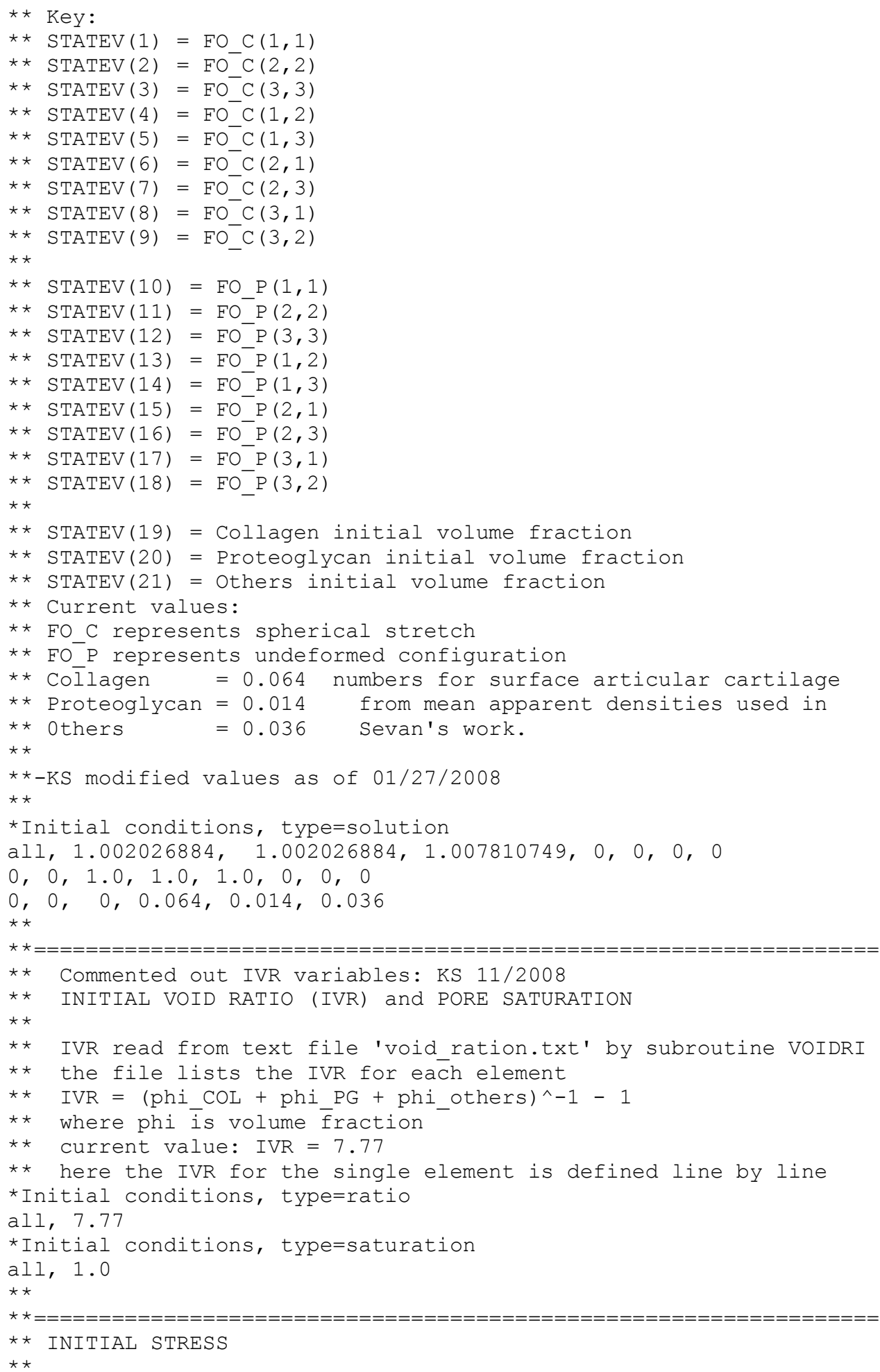




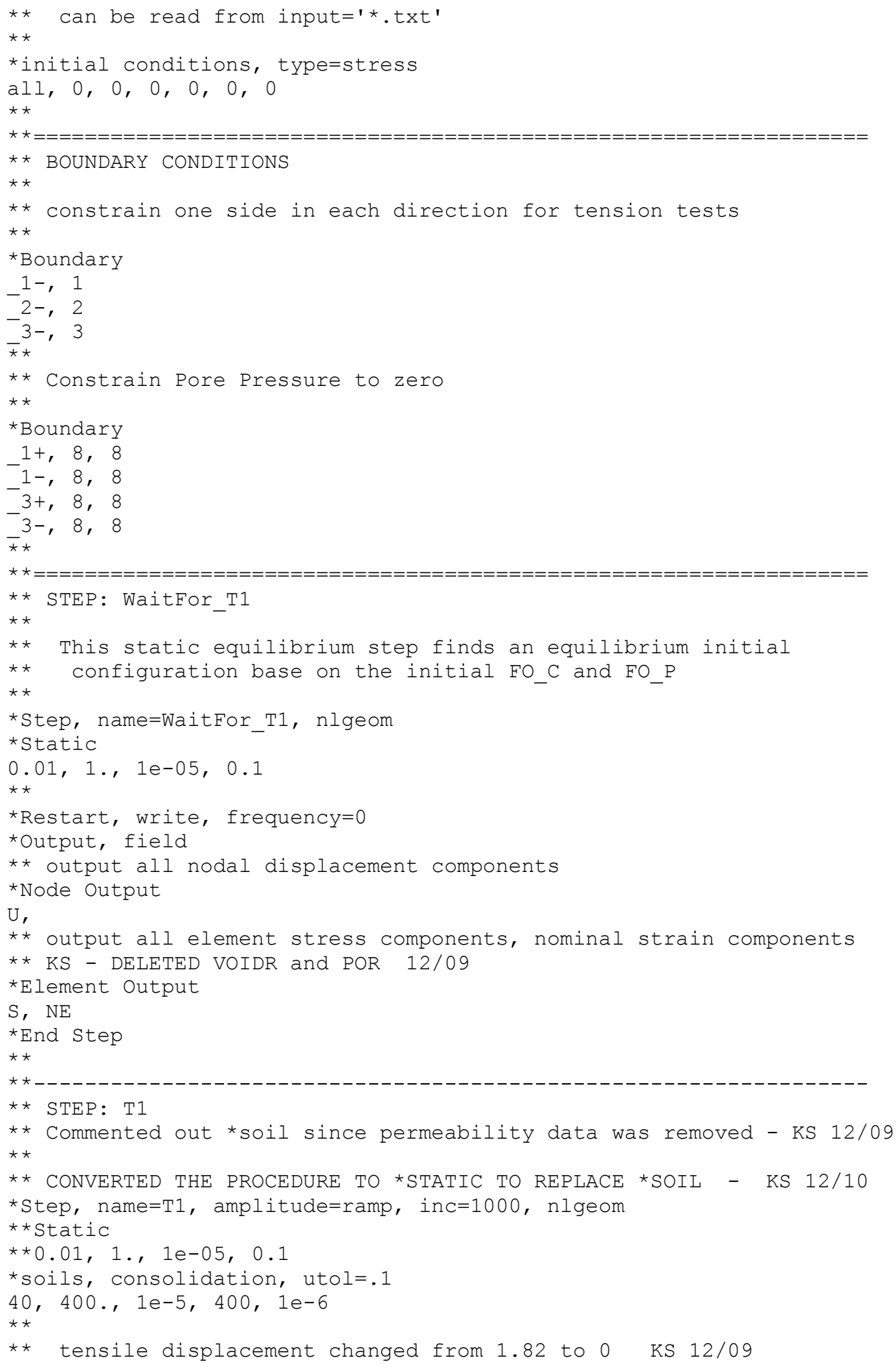




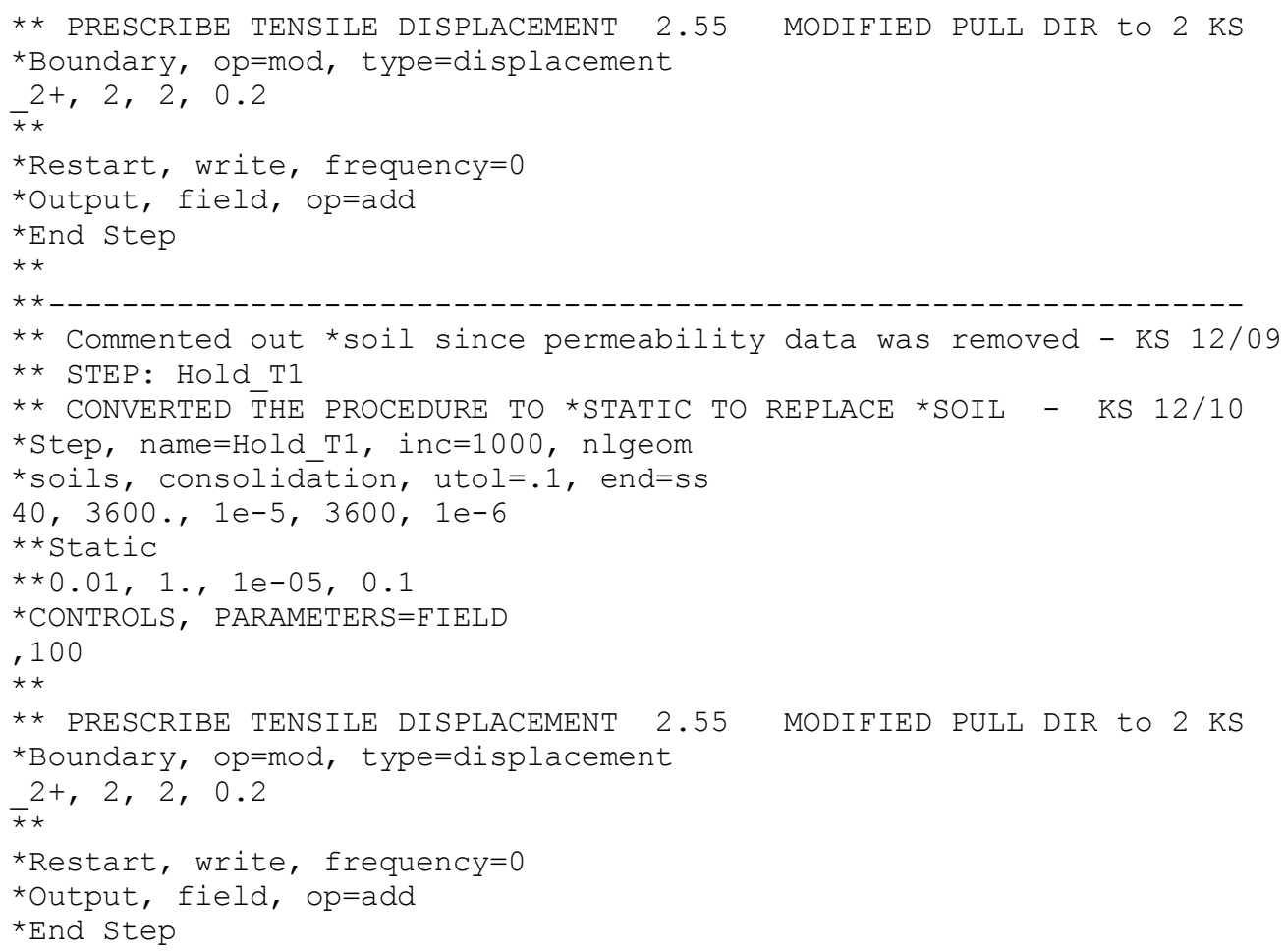

